SUPPLEMENTARY MATERIAL AVAILABLE

FOR

\title{
Addition of $\mathrm{H}_{2}$ on (sulfur, phosphorus, sulfur)-pincer-based Rhodium(I), Iridium(I), Palladium(II) and Platinum(II) Complexes: Reactivity and Regioselectivity
}

\author{
Marjolaine Doux, Louis Ricard, Pascal Le Floch,* and Yves Jean* \\ Laboratoire " Hétéroéléments et Coordination », UMR CNRS 7653, \\ Département de Chimie, Ecole Polytechnique, 91128 Palaiseau Cedex, France
}

\section{Computational details:}

Labelled picture, optimized geometryand lowest frequencies of $\left[\mathrm{Rh}(\mathrm{SPS})\left(\mathrm{PH}_{3}\right)\right] 1$ page Labelled picture, optimized geometry and lowest frequencies of $\left[\mathrm{Rh}(\mathrm{SPS})\left(\mathrm{PPh}_{3}\right)\right]$ at the ONIOM (B3PW91/UFF) level of theory 4 pages Labelled picture, optimized geometry and lowest frequencies of Ia 1 page Labelled picture, optimized geometry and lowest frequencies of Ia-rc 1 page Labelled picture, optimized geometry and lowest frequencies of IIa 1 page Labelled picture, optimized geometry and lowest frequencies of IIIa 1 page Labelled picture, optimized geometry and lowest frequencies of IVa 1 page Labelled picture, optimized geometry and lowest frequencies of Va 1 page Labelled picture, optimized geometry and lowest frequencies of VIa 1 page Labelled picture, optimized geometry and lowest frequencies of TS-Ia 1 page Labelled picture, optimized geometryand lowest frequencies of $\left[\operatorname{Ir}(\mathrm{SPS})\left(\mathrm{PH}_{3}\right)\right] 1$ page Labelled picture, optimized geometry and lowest frequencies of $\left[\operatorname{Ir}(\mathrm{SPS})\left(\mathrm{PPh}_{3}\right)\right]$ at the ONIOM (B3PW91/UFF) level of theory 7 pages Labelled picture, optimized geometry and lowest frequencies of Ib 1 page Labelled picture, optimized geometry and lowest frequencies of Ib-rc 1 page Labelled picture, optimized geometry and lowest frequencies of IIb 1 page Labelled picture, optimized geometry and lowest frequencies of IIIb 1 page Labelled picture, optimized geometry and lowest frequencies of IVb 1 page Labelled picture, optimized geometry and lowest frequencies of $\mathbf{V b} \quad 1$ page Labelled picture, optimized geometry and lowest frequencies of VIb 1 page Labelled picture, optimized geometry and lowest frequencies of TS-Ib 1 page 
Labelled picture, optimized geometryand lowest frequencies of $[\operatorname{Pd}(\mathrm{SPS})(\mathrm{Cl})] \quad 1$ page Labelled picture, optimized geometry and lowest frequencies of Ic 1 page

Labelled picture, optimized geometry and lowest frequencies of VIc 1 page

Labelled picture, optimized geometry and lowest frequencies of VIIc 1 page

Labelled picture, optimized geometryand lowest frequencies of $\left[\mathrm{Pd}(\mathrm{SPS})\left(\mathrm{PH}_{3}\right)\right]^{+} 1$ page

Labelled picture, optimized geometry and lowest frequencies of Id 1 page

Labelled picture, optimized geometry and lowest frequencies of VId 1 page

Labelled picture, optimized geometry and lowest frequencies of VIId 1 page

Labelled picture, optimized geometryand lowest frequencies of [ $\mathrm{Pt}(\mathrm{SPS})(\mathrm{Cl})] \quad 1$ page

Labelled picture, optimized geometry and lowest frequencies of Ie 1 page

Labelled picture, optimized geometry and lowest frequencies of VIe 1 page

Labelled picture, optimized geometry and lowest frequencies of VIIe 1 page

Labelled picture, optimized geometryand lowest frequencies of $\left[\mathrm{Pt}(\mathrm{SPS})\left(\mathrm{PH}_{3}\right)\right]^{+} 1$ page

Labelled picture, optimized geometry and lowest frequencies of If 1 page

Labelled picture, optimized geometry and lowest frequencies of VIf 1 page

\section{X-ray details:}

Table 1: Crystal data of compound $4 \quad 1$ page

Table 2: Atomic coordinates

and equivalent isotopic displacement parameters for $4 \quad 2$ pages

Table 3: Bond lengths and bond angles for $4 \quad 3$ pages

Table 4: Anisotropic displacement parameters for $4 \quad 2$ pages

Table 5: Hydrogen coordinates

and equivalent isotropic displacement parameters for $\mathbf{4} 1$ pages 


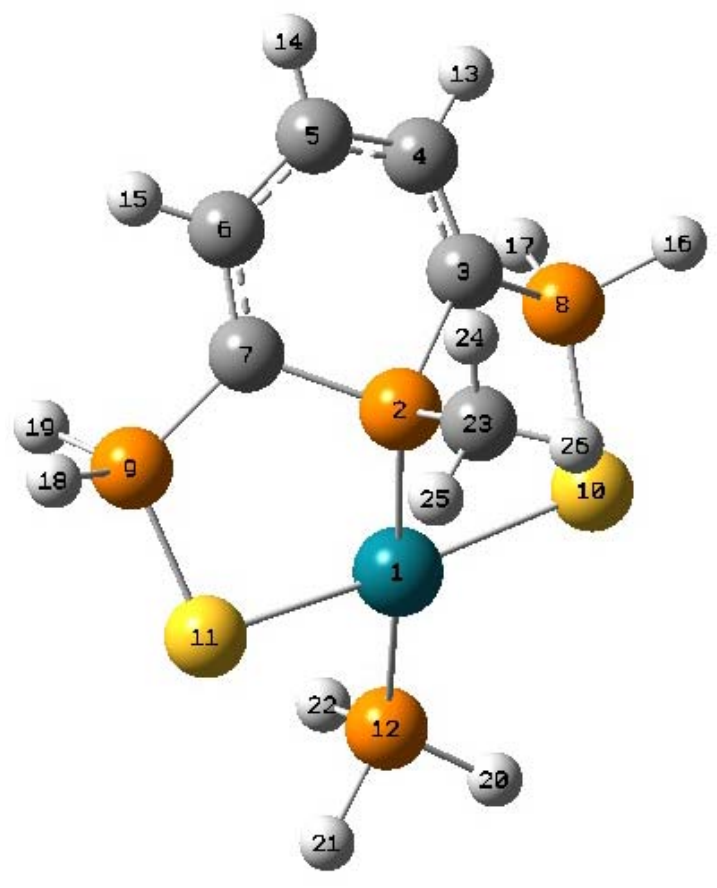

Optimized geometry:

\begin{tabular}{|c|c|c|c|c|c|}
\hline \multirow{2}{*}{$\begin{array}{l}\text { Center } \\
\text { Number }\end{array}$} & \multirow{2}{*}{$\begin{array}{l}\text { Atomic } \\
\text { Number }\end{array}$} & \multirow{2}{*}{$\begin{array}{l}\text { Atomic } \\
\text { Type }\end{array}$} & \multicolumn{3}{|c|}{ Coordinates (Angstroms) } \\
\hline & & & $\mathrm{X}$ & Y & Z \\
\hline 1 & 45 & 0 & -0.238747 & -0.342369 & -1.137165 \\
\hline 2 & 15 & 0 & -0.235853 & -0.338225 & 1.122302 \\
\hline 3 & 6 & 0 & 1.436756 & -0.345008 & 1.785088 \\
\hline 4 & 6 & 0 & 1.705614 & 0.296605 & 2.990255 \\
\hline 5 & 6 & 0 & 0.805759 & 1.155480 & 3.646373 \\
\hline 6 & 6 & 0 & -0.311210 & 1.703007 & 2.990255 \\
\hline 7 & 6 & 0 & -0.820348 & 1.228949 & 1.785088 \\
\hline 8 & 15 & 0 & 2.607163 & -0.930905 & 0.605629 \\
\hline 9 & 15 & 0 & -1.774680 & 2.124704 & 0.605628 \\
\hline 10 & 16 & 0 & 1.702767 & -1.749453 & -1.016977 \\
\hline 11 & 16 & 0 & -2.230190 & 0.993114 & -1.016968 \\
\hline 12 & 15 & 0 & -0.110616 & -0.158612 & -3.429022 \\
\hline 13 & 1 & 0 & 2.702219 & 0.208681 & 3.429483 \\
\hline 14 & 1 & 0 & 1.098864 & 1.575802 & 4.603492 \\
\hline 15 & 1 & 0 & -0.738186 & 2.607796 & 3.429482 \\
\hline 16 & 1 & 0 & 3.488317 & -1.868407 & 1.195475 \\
\hline 17 & 1 & 0 & 3.537129 & 0.084184 & 0.260741 \\
\hline 18 & 1 & 0 & -2.958958 & 2.627515 & 1.195478 \\
\hline 19 & 1 & 0 & -1.143588 & 3.348206 & 0.260727 \\
\hline 20 & 1 & 0 & 0.473587 & -1.176539 & -4.224665 \\
\hline 21 & 1 & 0 & -1.267740 & 0.037714 & -4.224658 \\
\hline 22 & 1 & 0 & 0.638039 & 0.915016 & -3.973566 \\
\hline 23 & 6 & 0 & -1.142853 & -1.638890 & 2.054502 \\
\hline 24 & 1 & 0 & -1.038830 & -1.489717 & 3.134814 \\
\hline 25 & 1 & 0 & -2.201348 & -1.599098 & 1.775660 \\
\hline 26 & 1 & 0 & -0.739650 & -2.618392 & 1.775661 \\
\hline
\end{tabular}

Basis set $\mathrm{A}$ :

SCF Done: $\mathrm{E}(\mathrm{RB}+\mathrm{HF}-\mathrm{PW} 91)=-2507.47087271$

Sum of electronic and zero-point Energies= Sum of electronic and thermal Energies= Sum of electronic and thermal Enthalpies= Sum of electronic and thermal Free Energies=
A.U. after 8 cycles

$-2507.297295$

$-2507.279105$

$-2507.278160$

$-2507.345320$

\section{E (Thermal) $\mathrm{KCal} / \mathrm{Mol}$}

120.336
$\mathrm{CV}$

Cal/Mol-Kelvin Cal/Mol-Kelvin 65.300 


\section{Frequencies:}

Frequencies -- 26.0361

Red. masses -- 2.7878

42.7847

Frc consts -- 0.0011

1. 2831

43.9851

$\begin{array}{lll}\text { FrC consts } & -- & 0.0011 \\ \text { IR Inten } & -- & 0.0062\end{array}$

0.0014

5.3800

0.0061

1. 8404 
Complex [Rh(SPS) $\left.\left(\mathrm{PPh}_{3}\right)\right]$ : Optimized structure of real complex [Rh (SPS) ( $\left.\mathrm{PPh}_{3}\right)$ ] obtained at the ONIOM (B3PW91/UFF) level of theory. Atoms included in the QM part are shown in ball and stick format and atoms included in the MM part are represented by tubes. Hydrogen atoms have been omitted for clarity.

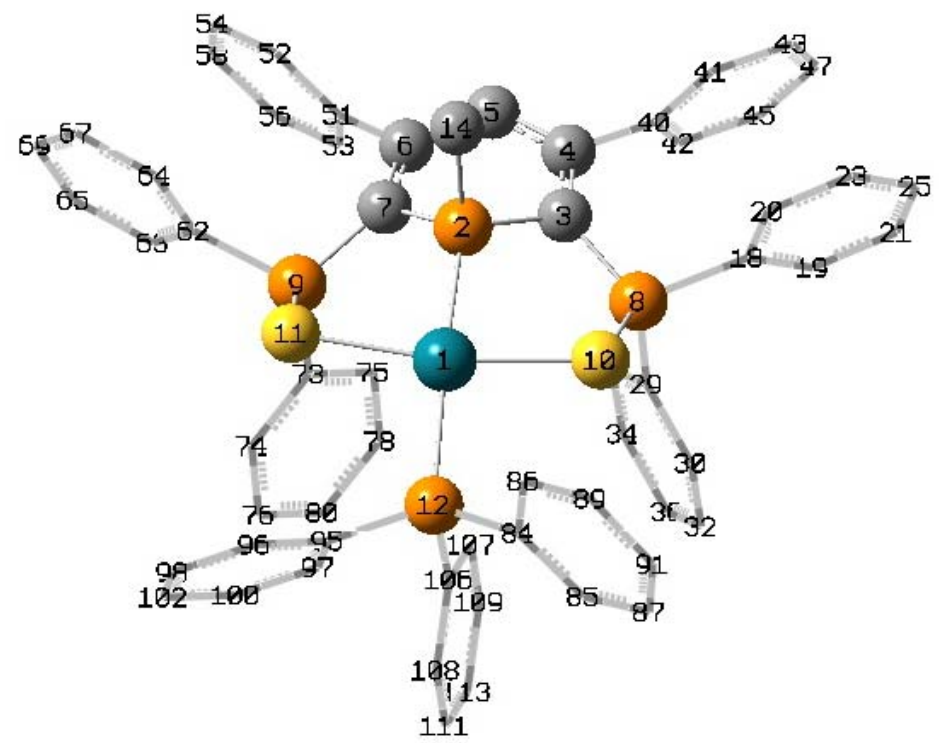

\begin{tabular}{|c|c|c|c|c|c|}
\hline \multirow{2}{*}{$\begin{array}{l}\text { Center } \\
\text { Number }\end{array}$} & \multirow{2}{*}{$\begin{array}{l}\text { Atomic } \\
\text { Number }\end{array}$} & \multirow{2}{*}{$\begin{array}{c}\text { Atomic } \\
\text { Type }\end{array}$} & \multicolumn{3}{|c|}{ Coordinates (Angstroms) } \\
\hline & & & $\mathrm{X}$ & Y & Z \\
\hline - & & & 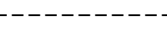 & --------1 & ---------- \\
\hline 1 & 45 & 0 & 1.237186 & -.071159 & -1.104968 \\
\hline 2 & 15 & 0 & -.964851 & .051822 & -1.582300 \\
\hline 3 & 6 & 0 & -1.945875 & -1.239536 & -.804369 \\
\hline 4 & 6 & 0 & -3.316295 & -.988658 & -.617984 \\
\hline 5 & 6 & 0 & -3.864184 & .305937 & -.696265 \\
\hline 6 & 6 & 0 & -3.107428 & 1.499154 & -.644323 \\
\hline 7 & 6 & 0 & -1.730109 & 1.522034 & -.873579 \\
\hline 8 & 15 & 0 & -.897476 & -2.543193 & -.213291 \\
\hline 9 & 15 & 0 & -.459299 & 2.677787 & -.407671 \\
\hline 10 & 16 & 0 & .941620 & -2.442017 & -1.086330 \\
\hline 11 & 16 & 0 & 1.257726 & 2.293775 & -1.431677 \\
\hline 12 & 15 & 0 & 3.423678 & -.270307 & -.318171 \\
\hline 13 & 1 & 0 & -4.947310 & .398332 & -.626203 \\
\hline 14 & 6 & 0 & -1.506003 & .055639 & -3.340104 \\
\hline 15 & 1 & 0 & -2.595356 & .139557 & -3.415454 \\
\hline 16 & 1 & 0 & -1.031897 & .902367 & -3.847629 \\
\hline 17 & 1 & 0 & -1.172034 & -.875691 & -3.809735 \\
\hline 18 & 6 & 0 & -1.626177 & -4.147620 & -.606603 \\
\hline 19 & 6 & 0 & -2.290517 & -4.905818 & .372948 \\
\hline 20 & 6 & 0 & -1.557506 & -4.631771 & -1.921384 \\
\hline 21 & 6 & 0 & -2.863523 & -6.136858 & .040287 \\
\hline 22 & 1 & 0 & -2.385720 & -4.545109 & 1.387787 \\
\hline 23 & 6 & 0 & -2.134567 & -5.861777 & -2.250697 \\
\hline 24 & 1 & 0 & -1.067245 & -4.052991 & -2.693817 \\
\hline 25 & 6 & 0 & -2.785383 & -6.614925 & -1.270179 \\
\hline 26 & 1 & 0 & -3.378415 & -6.714950 & .796602 \\
\hline 27 & 1 & 0 & -2.080823 & -6.229094 & -3.267248 \\
\hline 28 & 1 & 0 & -3.234860 & -7.565291 & -1.527049 \\
\hline 29 & 6 & 0 & -.684075 & -2.510081 & 1.580198 \\
\hline 30 & 6 & 0 & .280270 & -3.335737 & 2.177748 \\
\hline 31 & 6 & 0 & -1.496713 & -1.702741 & 2.388987 \\
\hline 32 & 6 & 0 & .422784 & -3.359589 & 3.568048 \\
\hline 33 & 1 & 0 & .913156 & -3.968850 & 1.569006 \\
\hline 34 & 6 & 0 & -1.357074 & -1.733906 & 3.779366 \\
\hline 35 & 1 & 0 & -2.241906 & -1.055915 & 1.953000 \\
\hline 36 & 6 & 0 & -.399966 & -2.563650 & 4.369180 \\
\hline 37 & 1 & 0 & 1.167618 & -3.998339 & 4.024488 \\
\hline 38 & 1 & 0 & -1.992601 & -1.116181 & 4.400112 \\
\hline 39 & 1 & 0 & -.291207 & -2.584267 & 5.445702 \\
\hline
\end{tabular}




\begin{tabular}{|c|c|c|c|c|c|}
\hline 40 & 6 & 0 & -4.246289 & -2.101093 & -.315861 \\
\hline 41 & 6 & 0 & -4.561611 & -3.038327 & -1.311668 \\
\hline 42 & 6 & 0 & -4.848971 & -2.204015 & .947571 \\
\hline 43 & 6 & 0 & -5.446894 & -4.084698 & -1.036617 \\
\hline 44 & 1 & 0 & -4.117153 & -2.960617 & -2.296404 \\
\hline 45 & 6 & 0 & -5.728894 & -3.255686 & 1.221039 \\
\hline 46 & 1 & 0 & -4.634953 & -1.475347 & 1.719112 \\
\hline 47 & 6 & 0 & -6.025270 & -4.197074 & .230836 \\
\hline 48 & 1 & 0 & -5.678626 & -4.812137 & -1.803705 \\
\hline 49 & 1 & 0 & -6.183557 & -3.339330 & 2.199535 \\
\hline 50 & 1 & 0 & -6.706085 & -5.010824 & .444144 \\
\hline 51 & 6 & 0 & -3.830129 & 2.743028 & -.298463 \\
\hline 52 & 6 & 0 & -4.135953 & 3.676660 & -1.300071 \\
\hline 53 & 6 & 0 & -4.242157 & 2.981216 & 1.021445 \\
\hline 54 & 6 & 0 & -4.825797 & 4.849469 & -.978919 \\
\hline 55 & 1 & 0 & -3.833219 & 3.498590 & -2.324686 \\
\hline 56 & 6 & 0 & -4.926778 & 4.158003 & 1.339998 \\
\hline 57 & 1 & 0 & -4.030282 & 2.260543 & 1.801042 \\
\hline 58 & 6 & 0 & -5.216523 & 5.092466 & .341150 \\
\hline 59 & 1 & 0 & -5.050505 & 5.573066 & -1.751630 \\
\hline 60 & 1 & 0 & -5.234503 & 4.344670 & 2.360614 \\
\hline 61 & 1 & 0 & -5.744934 & 6.003620 & .589584 \\
\hline 62 & 6 & 0 & -1.000467 & 4.359630 & -.779395 \\
\hline 63 & 6 & 0 & -1.465325 & 5.215674 & .233606 \\
\hline 64 & 6 & 0 & -.986790 & 4.809901 & -2.107771 \\
\hline 65 & 6 & 0 & -1.895478 & 6.508186 & -.080714 \\
\hline 66 & 1 & 0 & -1.515081 & 4.886273 & 1.262246 \\
\hline 67 & 6 & 0 & -1.421146 & 6.101876 & -2.418594 \\
\hline 68 & 1 & 0 & -.650176 & 4.158165 & -2.903986 \\
\hline 69 & 6 & 0 & -1.873188 & 6.951365 & -1.405527 \\
\hline 70 & 1 & 0 & -2.257212 & 7.162313 & .701924 \\
\hline 71 & 1 & 0 & -1.411071 & 6.442998 & -3.445603 \\
\hline 72 & 1 & 0 & -2.212450 & 7.950058 & -1.647769 \\
\hline 73 & 6 & 0 & -.104821 & 2.625333 & 1.361010 \\
\hline 74 & 6 & 0 & .978145 & 3.355220 & 1.872354 \\
\hline 75 & 6 & 0 & -.917173 & 1.891393 & 2.237089 \\
\hline 76 & 6 & 0 & 1.247460 & 3.348008 & 3.243770 \\
\hline 77 & 1 & 0 & 1.604507 & 3.940279 & 1.211337 \\
\hline 78 & 6 & 0 & -.654164 & 1.896912 & 3.609872 \\
\hline 79 & 1 & 0 & -1.755345 & 1.321672 & 1.866236 \\
\hline 80 & 6 & 0 & .428245 & 2.623456 & 4.113211 \\
\hline 81 & 1 & 0 & 2.086040 & 3.910411 & 3.633165 \\
\hline 82 & 1 & 0 & -1.287383 & 1.335962 & 4.284013 \\
\hline 83 & 1 & 0 & .634879 & 2.620505 & 5.175490 \\
\hline 84 & 6 & 0 & 4.218027 & -1.823944 & -.866312 \\
\hline 85 & 6 & 0 & 4.628181 & -2.822451 & .028583 \\
\hline 86 & 6 & 0 & 4.309020 & -2.101627 & -2.315372 \\
\hline 87 & 6 & 0 & 5.163218 & -4.022586 & -.450149 \\
\hline 88 & 1 & 0 & 4.524941 & -2.697891 & 1.095456 \\
\hline 89 & 6 & 0 & 4.848334 & -3.310331 & -2.751630 \\
\hline 90 & 1 & 0 & 3.961602 & -1.372563 & -3.036282 \\
\hline 91 & 6 & 0 & 5.278034 & -4.261497 & -1.823625 \\
\hline 92 & 1 & 0 & 5.483291 & -4.781928 & .251741 \\
\hline 93 & 1 & 0 & 4.928530 & -3.514529 & -3.811395 \\
\hline 94 & 1 & 0 & 5.693187 & -5.198774 & -2.171077 \\
\hline 95 & 6 & 0 & 4.578654 & 1.099395 & -.694952 \\
\hline 96 & 6 & 0 & 4.346388 & 2.358777 & -.121316 \\
\hline 97 & 6 & 0 & 5.710428 & .917493 & -1.508272 \\
\hline 98 & 6 & 0 & 5.211508 & 3.425856 & -.376844 \\
\hline 99 & 1 & 0 & 3.500474 & 2.510510 & .534743 \\
\hline 100 & 6 & 0 & 6.575042 & 1.987197 & -1.762159 \\
\hline 101 & 1 & 0 & 5.939598 & -.041165 & -1.945586 \\
\hline 102 & 6 & 0 & 6.324085 & 3.241230 & -1.200247 \\
\hline 103 & 1 & 0 & 5.021657 & 4.394178 & .067877 \\
\hline 104 & 1 & 0 & 7.442955 & 1.842491 & -2.392515 \\
\hline 105 & 1 & 0 & 6.995046 & 4.067307 & -1.397309 \\
\hline 106 & 6 & 0 & 3.445624 & -.300333 & 1.499490 \\
\hline 107 & 6 & 0 & 2.173533 & -.281380 & 2.243069 \\
\hline 108 & 6 & 0 & 4.647979 & -.264884 & 2.214423 \\
\hline 109 & 6 & 0 & 2.202130 & -.259750 & 3.636684 \\
\hline 110 & 1 & 0 & 1.228412 & -.276860 & 1.716264 \\
\hline 111 & 6 & 0 & 4.633318 & -.243499 & 3.611957 \\
\hline 112 & 1 & 0 & 5.595511 & -.257265 & 1.690792 \\
\hline 113 & 6 & 0 & 3.423428 & -.243231 & 4.315498 \\
\hline 114 & 1 & 0 & 1.277271 & -.247436 & 4.195945 \\
\hline 115 & 1 & 0 & 5.568641 & -.223022 & 4.156095 \\
\hline 116 & 1 & 0 & 3.433167 & -.223733 & 5.397516 \\
\hline
\end{tabular}


Basis set $A$ :

Frequencies:

1

Frequencies -- $\quad 11.3097$

A

Red. masses -- 6.1590

16.2835

5.8007

.0009

A

Frc consts -- $\quad .0005$

IR Inten -- $\quad .1085$

.0665

19.4906

5.7964

.0013

.0338

Basis set $B$ :

$\mathrm{SCF}$ Done: $\mathrm{E}(\mathrm{RB}+\mathrm{HF}-\mathrm{PW} 91)=-4585.74934902$

A.U. after 26 cycles 
$\left[\mathrm{Rh}\left(\mathrm{SPS}\left(\mathrm{PH}_{3}\right)(\mathrm{H})_{2}\right]\right.$ syn-SS (Ia)

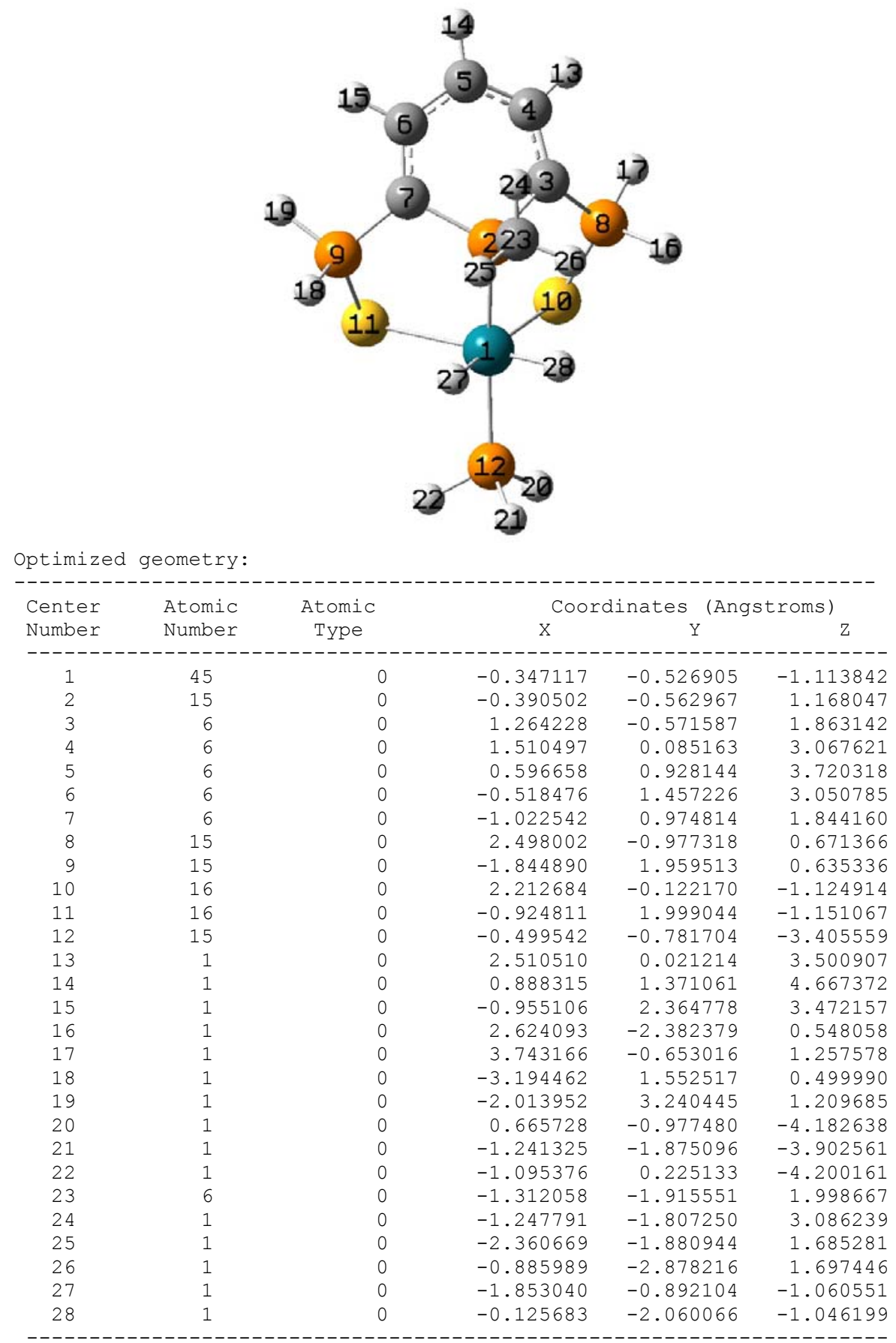

Basis set $A$ :

Selected geometrical parameters: H-H 2.085, Rh-H 1.550, Rh-S 2.591, Rh-P1 2.283, Rh-P4 2.311, S1-Rh-S2 93.9, P1-Rh-P4 171.3.

$\mathrm{SCF}$ Done: $\mathrm{E}(\mathrm{RB}+\mathrm{HF}-\mathrm{PW} 91)=-2508.67235653$

Sum of electronic and zero-point Energies=

Sum of electronic and thermal Energies=

Sum of electronic and thermal Enthalpies=

Sum of electronic and thermal Free Energies=

\section{A.U. after 7 cycles}

$-2508.481091$

$-2508.462709$

$-2508.461765$

$-2508.527927$

$$
\begin{gathered}
\text { E (Thermal) } \\
\text { KCal/Mol }
\end{gathered}
$$

Total
CV

$\mathrm{Cal} / \mathrm{Mol}-\mathrm{Kelvin}$ 67.855
$\mathrm{Cal} / \mathrm{Mol}-\mathrm{Kelvin}$ 139.249

Frequencies: 
Frequencies -- $\quad 44.9461$

Red. masses -- 1.6439

Frc consts -- 0.0020

IR Inten -- $\quad 0.2993$
53.9801

2.0290

0.0035

0.1194
65.3952

5.0299

0.0127

0.2495 
[Rh (SPS $\left.\left(\mathrm{PH}_{3}\right)\left(\mathrm{H}_{2}\right)_{2}\right]$ syn-SS (Ia-rc) : Optimized structure of real complex [Rh(SPS) $\left.\left(\mathrm{PPh}_{3}\right)\right]$ obtained at the ONIOM (B3PW91/UFF) level of theory. Atoms included in the $Q M$ part are shown in ball and stick format and atoms included in the MM part are represented by tubes.

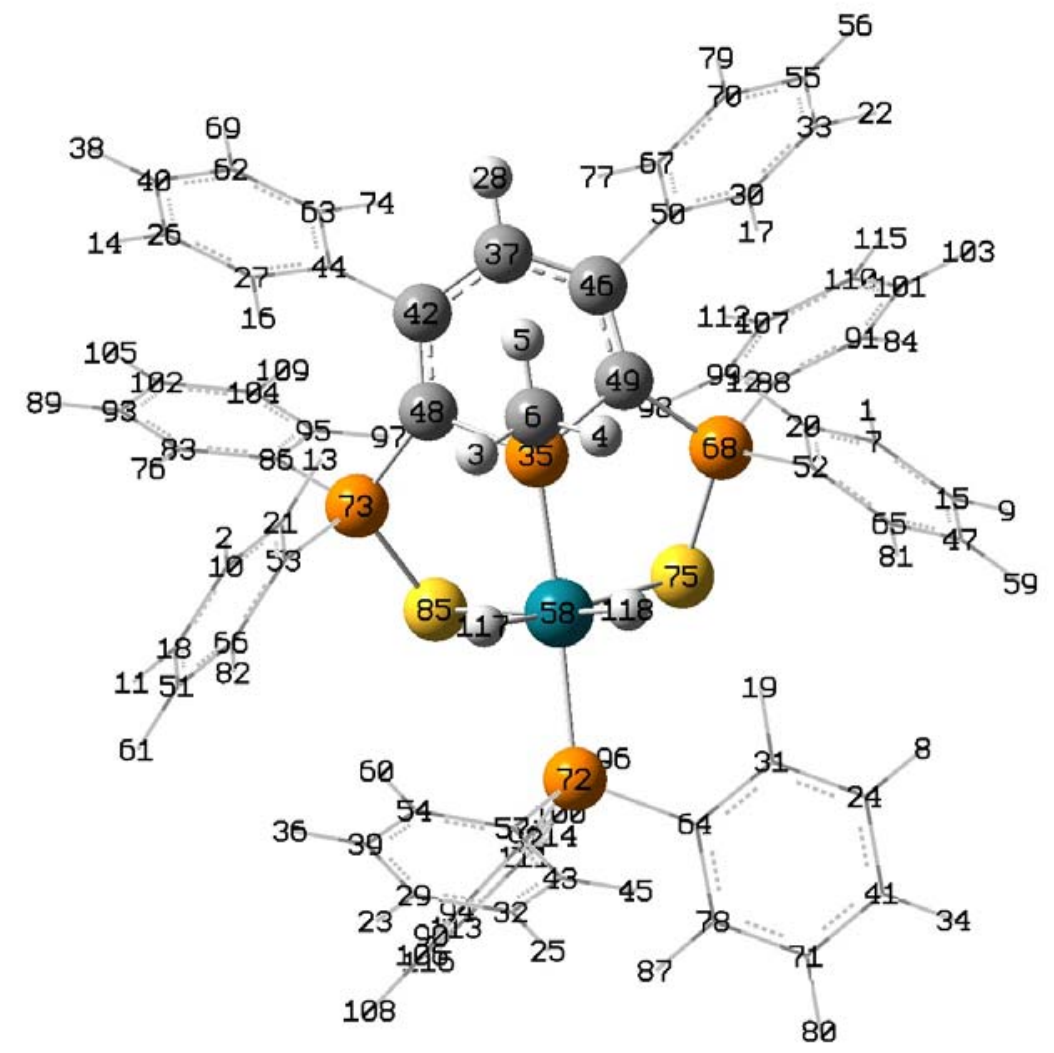

Optimized geometry:

\begin{tabular}{|c|c|c|c|c|c|}
\hline \multirow{2}{*}{$\begin{array}{l}\text { Center } \\
\text { Number }\end{array}$} & \multirow{2}{*}{$\begin{array}{l}\text { Atomic } \\
\text { Number }\end{array}$} & \multirow{2}{*}{$\begin{array}{c}\text { Atomic } \\
\text { Type }\end{array}$} & \multicolumn{3}{|c|}{ Coordinates (Angstroms) } \\
\hline & & & $\mathrm{X}$ & $\mathrm{Y}$ & Z \\
\hline & & & & 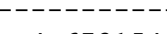 & ---------- \\
\hline 1 & 1 & 0 & -0.624404 & -4.673154 & -4.257197 \\
\hline 2 & 1 & 0 & 1.294039 & 4.374886 & -4.122557 \\
\hline 3 & 1 & 0 & -0.136751 & 0.859114 & -3.481647 \\
\hline 4 & 1 & 0 & -0.421312 & -0.884706 & -3.479056 \\
\hline 5 & 1 & 0 & -1.817501 & 0.238811 & -3.469819 \\
\hline 6 & 6 & 0 & -0.798031 & 0.072516 & -3.108011 \\
\hline 7 & 6 & 0 & -0.465132 & -4.740302 & -3.188753 \\
\hline 8 & 1 & 0 & 3.156180 & -5.432216 & -0.182889 \\
\hline 9 & 1 & 0 & 0.557855 & -6.631700 & -3.301756 \\
\hline 10 & 6 & 0 & 1.417178 & 4.364638 & -3.047320 \\
\hline 11 & 1 & 0 & 3.136386 & 5.660858 & -3.074539 \\
\hline 12 & 1 & 0 & -1.439972 & -2.879393 & -2.784944 \\
\hline 13 & 1 & 0 & -0.257907 & 3.072437 & -2.726333 \\
\hline 14 & 1 & 0 & -3.532286 & 6.166474 & -2.215048 \\
\hline 15 & 6 & 0 & 0.201275 & -5.844185 & -2.650613 \\
\hline 16 & 1 & 0 & -2.405396 & 3.981319 & -2.398497 \\
\hline 17 & 1 & 0 & -3.747585 & -3.010927 & -2.403079 \\
\hline 18 & 6 & 0 & 2.454930 & 5.090412 & -2.456905 \\
\hline 19 & 1 & 0 & 2.343280 & -3.118468 & -0.407639 \\
\hline 20 & 6 & 0 & -0.926787 & -3.722387 & -2.349129 \\
\hline 21 & 6 & 0 & 0.538130 & 3.624372 & -2.251330 \\
\hline 22 & 1 & 0 & -5.568158 & -4.654787 & -2.155830 \\
\hline 23 & 1 & 0 & 6.742252 & 2.164263 & -3.598158 \\
\hline 24 & 6 & 0 & 3.778761 & -4.608931 & 0.140802 \\
\hline 25 & 1 & 0 & 7.041186 & -0.268849 & -3.232663 \\
\hline 26 & 6 & 0 & -3.845817 & 5.344696 & -1.584495 \\
\hline 27 & 6 & 0 & -3.203806 & 4.106490 & -1.681236 \\
\hline 28 & 1 & 0 & -4.771439 & 0.774888 & -1.426557 \\
\hline 29 & 6 & 0 & 6.144525 & 1.651012 & -2.856115 \\
\hline 30 & 6 & 0 & -4.499646 & -2.857037 & -1.643099 \\
\hline 31 & 6 & 0 & 3.319923 & -3.293993 & 0.016118 \\
\hline
\end{tabular}




\begin{tabular}{|c|c|c|c|c|c|}
\hline 32 & 6 & 0 & 6.314884 & 0.279959 & -2.647203 \\
\hline 33 & 6 & 0 & -5.535931 & -3.786273 & -1.511008 \\
\hline 34 & 1 & 0 & 5.394516 & -5.880832 & 0.779682 \\
\hline 35 & 15 & 0 & -0.755417 & 0.068658 & -1.271273 \\
\hline 36 & 1 & 0 & 5.058674 & 3.417743 & -2.279419 \\
\hline 37 & 6 & 0 & -3.722219 & 0.593065 & -1.195883 \\
\hline 38 & 1 & 0 & -5.380969 & 6.484331 & -0.594583 \\
\hline 39 & 6 & 0 & 5.195991 & 2.357260 & -2.113222 \\
\hline 40 & 6 & 0 & -4.889112 & 5.523499 & -0.672337 \\
\hline 41 & 6 & 0 & 5.041311 & -4.862330 & 0.683202 \\
\hline 42 & 6 & 0 & -2.913057 & 1.722892 & -0.954796 \\
\hline 43 & 6 & 0 & 5.541471 & -0.386892 & -1.691424 \\
\hline 44 & 6 & 0 & -3.599772 & 3.037432 & -0.860927 \\
\hline 45 & 1 & 0 & 5.676010 & -1.451669 & -1.571229 \\
\hline 46 & 6 & 0 & -3.346488 & -0.743128 & -0.937982 \\
\hline 47 & 6 & 0 & 0.406782 & -5.931033 & -1.271554 \\
\hline 48 & 6 & 0 & -1.522496 & 1.622303 & -0.777797 \\
\hline 49 & 6 & 0 & -2.006191 & -1.129097 & -0.770637 \\
\hline 50 & 6 & 0 & -4.447436 & -1.731478 & -0.805174 \\
\hline 51 & 6 & 0 & 2.613432 & 5.078346 & -1.068888 \\
\hline 52 & 6 & 0 & -0.724444 & -3.803152 & -0.963638 \\
\hline 53 & 6 & 0 & 0.694969 & 3.602955 & -0.857936 \\
\hline 54 & 6 & 0 & 4.422009 & 1.693945 & -1.157639 \\
\hline 55 & 6 & 0 & -6.529103 & -3.595828 & -0.546574 \\
\hline 56 & 1 & 0 & -7.327528 & -4.318842 & -0.442057 \\
\hline 57 & 6 & 0 & 4.599250 & 0.321153 & -0.925612 \\
\hline 58 & 45 & 0 & 1.268682 & -0.259832 & -0.309639 \\
\hline 59 & 1 & 0 & 0.922897 & -6.785989 & -0.854414 \\
\hline 60 & 1 & 0 & 3.688238 & 2.250312 & -0.590003 \\
\hline 61 & 1 & 0 & 3.418482 & 5.638628 & -0.611465 \\
\hline 62 & 6 & 0 & -5.297791 & 4.461855 & 0.138986 \\
\hline 63 & 6 & 0 & -4.661372 & 3.220985 & 0.042466 \\
\hline 64 & 6 & 0 & 4.123669 & -2.223820 & 0.433123 \\
\hline 65 & 6 & 0 & -0.050997 & -4.912973 & -0.430270 \\
\hline 66 & 6 & 0 & 1.737083 & 4.337138 & -0.271088 \\
\hline 67 & 6 & 0 & -5.459805 & -1.538073 & 0.150759 \\
\hline 68 & 15 & 0 & -1.297241 & -2.492950 & 0.141810 \\
\hline 69 & 1 & 0 & -6.104809 & 4.601638 & 0.846292 \\
\hline 70 & 6 & 0 & -6.492223 & -2.471333 & 0.282056 \\
\hline 71 & 6 & 0 & 5.847967 & -3.800381 & 1.100177 \\
\hline 72 & 15 & 0 & 3.522874 & -0.508262 & 0.294590 \\
\hline 73 & 15 & 0 & -0.405706 & 2.626122 & 0.190249 \\
\hline 74 & 1 & 0 & -4.980370 & 2.409895 & 0.685627 \\
\hline 75 & 16 & 0 & 0.256527 & -1.948019 & 1.306141 \\
\hline 76 & 1 & 0 & -0.879976 & 5.555707 & 0.040876 \\
\hline 77 & 1 & 0 & -5.437313 & -0.675600 & 0.805724 \\
\hline 78 & 6 & 0 & 5.391929 & -2.484757 & 0.976757 \\
\hline 79 & 1 & 0 & -7.261251 & -2.324932 & 1.029272 \\
\hline 80 & 1 & 0 & 6.826212 & -3.996550 & 1.519559 \\
\hline 81 & 1 & 0 & 0.116718 & -4.990853 & 0.636514 \\
\hline 82 & 1 & 0 & 1.872868 & 4.332656 & 0.802998 \\
\hline 83 & 6 & 0 & -1.415583 & 5.152262 & 0.888712 \\
\hline 84 & 1 & 0 & -2.862195 & -5.021450 & 0.062142 \\
\hline 85 & 16 & 0 & 0.742102 & 1.526634 & 1.430138 \\
\hline 86 & 6 & 0 & -1.366041 & 3.781194 & 1.192433 \\
\hline 87 & 1 & 0 & 6.027600 & -1.670867 & 1.301347 \\
\hline 88 & 6 & 0 & -2.567848 & -3.203167 & 1.210957 \\
\hline 89 & 1 & 0 & -2.231485 & 7.071130 & 1.425678 \\
\hline 90 & 1 & 0 & 5.554741 & 1.488776 & 1.263684 \\
\hline 91 & 6 & 0 & -3.153605 & -4.449707 & 0.931795 \\
\hline 92 & 6 & 0 & 3.938006 & 0.197715 & 1.929900 \\
\hline 93 & 6 & 0 & -2.188795 & 6.017408 & 1.668684 \\
\hline 94 & 6 & 0 & 4.960455 & 1.148539 & 2.097617 \\
\hline 95 & 6 & 0 & -2.099389 & 3.291953 & 2.283967 \\
\hline 96 & 1 & 0 & 2.459989 & -1.000331 & 2.953659 \\
\hline 97 & 1 & 0 & -2.084364 & 2.236266 & 2.524574 \\
\hline 98 & 1 & 0 & -2.569351 & -1.508268 & 2.554148 \\
\hline 99 & 6 & 0 & -2.998625 & -2.477215 & 2.331676 \\
\hline 100 & 6 & 0 & 3.232336 & -0.251271 & 3.055906 \\
\hline 101 & 6 & 0 & -4.152791 & -4.959570 & 1.766239 \\
\hline 102 & 6 & 0 & -2.916680 & 5.522146 & 2.753476 \\
\hline 103 & 1 & 0 & -4.607336 & -5.915901 & 1.542413 \\
\hline 104 & 6 & 0 & -2.871931 & 4.160064 & 3.060835 \\
\hline 105 & 1 & 0 & -3.519237 & 6.192674 & 3.352229 \\
\hline 106 & 6 & 0 & 5.244201 & 1.661129 & 3.367301 \\
\hline 107 & 6 & 0 & -3.998787 & -2.989783 & 3.162808 \\
\hline 108 & 1 & 0 & 6.030409 & 2.394813 & 3.490283 \\
\hline
\end{tabular}




$\begin{array}{rrrrrr}109 & 1 & 0 & -3.440705 & 3.775650 & 3.897395 \\ 110 & 6 & 0 & -4.575330 & -4.230588 & 2.880611 \\ 111 & 6 & 0 & 3.517538 & 0.263837 & 4.323360 \\ 112 & 1 & 0 & -4.330779 & -2.422205 & 4.022364 \\ 113 & 6 & 0 & 4.520367 & 1.223093 & 4.478679 \\ 114 & 1 & 0 & 2.962464 & -0.082984 & 5.185331 \\ 115 & 1 & 0 & -5.353242 & -4.624378 & 3.521674 \\ 116 & 1 & 0 & 4.741848 & 1.620799 & 5.460536 \\ 117 & 1 & 0 & 1.766035 & 0.770586 & -1.359048 \\ 118 & 1 & 0 & 1.536480 & -1.263206 & -1.465774 \\ - & 1 & & \end{array}$

Basis set $A$ :

Frequencies:

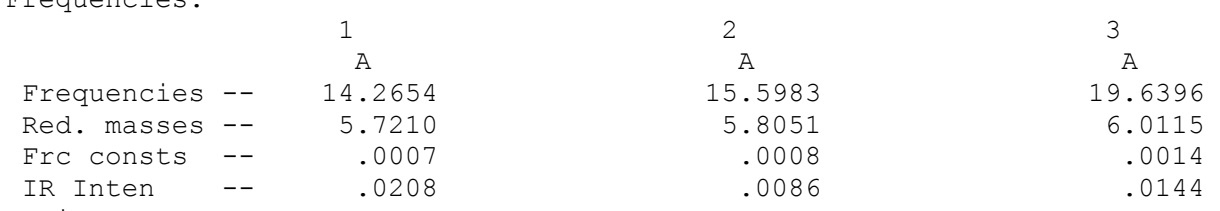

Basis set $B$ :

$\mathrm{SCF}$ Done: $\mathrm{E}(\mathrm{RB}+\mathrm{HF}-\mathrm{PW} 91)=-4586.95979769$

A.U. after 23 cycles 
[Rh $\left(\mathrm{SPS}\left(\mathrm{PH}_{3}\right)(\mathrm{H})_{2}\right]$ syn-PP (IIa)

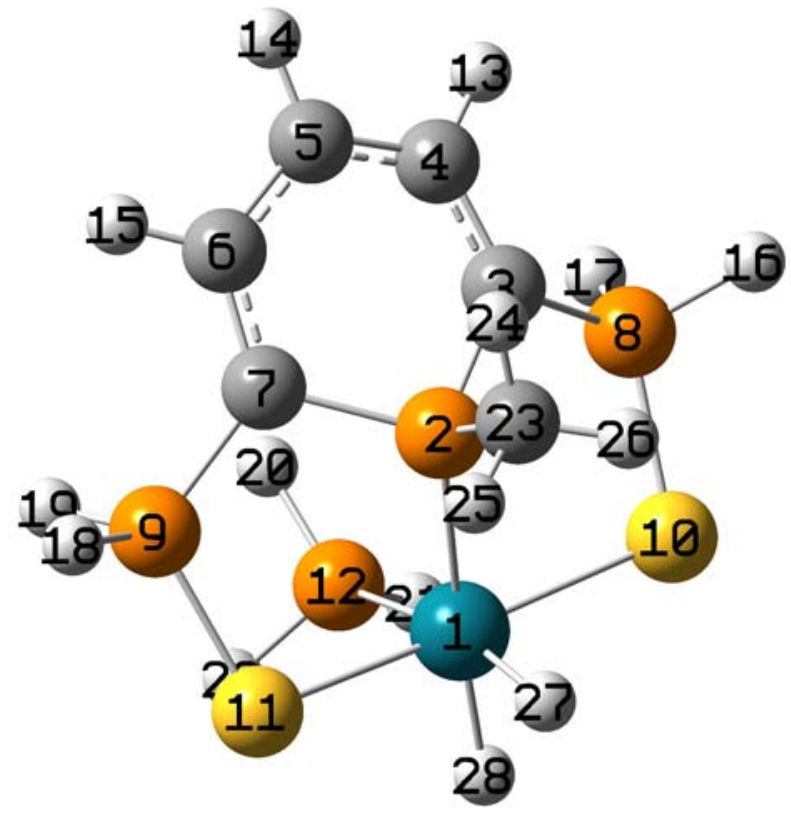

Optimized geometry:

\begin{tabular}{|c|c|c|c|c|c|}
\hline \multirow{2}{*}{$\begin{array}{l}\text { Center } \\
\text { Number }\end{array}$} & \multirow{2}{*}{$\begin{array}{l}\text { Atomic } \\
\text { Number }\end{array}$} & \multirow{2}{*}{$\begin{array}{l}\text { Atomic } \\
\text { Type }\end{array}$} & \multicolumn{3}{|c|}{ Coordinates (Angstroms) } \\
\hline & & & $\mathrm{X}$ & Y & Z \\
\hline & & & -------1 & ---------1 & $-1------$ \\
\hline 1 & 45 & 0 & -0.339552 & -0.480472 & -1.302531 \\
\hline 2 & 15 & 0 & -0.332565 & -0.479149 & 1.027563 \\
\hline 3 & 6 & 0 & 1.353221 & -0.492721 & 1.653302 \\
\hline 4 & 6 & 0 & 1.686215 & 0.250782 & 2.782579 \\
\hline 5 & 6 & 0 & 0.834253 & 1.183633 & 3.399846 \\
\hline 6 & 6 & 0 & -0.331337 & 1.669983 & 2.780260 \\
\hline 7 & 6 & 0 & -0.916987 & 1.103511 & 1.652280 \\
\hline 8 & 15 & 0 & 2.476756 & -1.268925 & 0.534890 \\
\hline 9 & 15 & 0 & -2.025695 & 1.895554 & 0.528585 \\
\hline 10 & 16 & 0 & 1.583909 & -1.937517 & -1.179510 \\
\hline 11 & 16 & 0 & -2.381585 & 0.802583 & -1.161825 \\
\hline 12 & 15 & 0 & 0.985463 & 1.422504 & -1.982569 \\
\hline 13 & 1 & 0 & 2.697220 & 0.177621 & 3.190159 \\
\hline 14 & 1 & 0 & 1.186770 & 1.685950 & 4.295290 \\
\hline 15 & 1 & 0 & -0.740674 & 2.598811 & 3.184541 \\
\hline 16 & 1 & 0 & 3.151327 & -2.363009 & 1.125359 \\
\hline 17 & 1 & 0 & 3.590370 & -0.415759 & 0.304529 \\
\hline 18 & 1 & 0 & -3.272052 & 2.191071 & 1.128010 \\
\hline 19 & 1 & 0 & -1.580483 & 3.219933 & 0.266074 \\
\hline 20 & 1 & 0 & 1.682157 & 2.406153 & -1.218938 \\
\hline 21 & 1 & 0 & 2.067919 & 1.120910 & -2.841434 \\
\hline 22 & 1 & 0 & 0.335465 & 2.344524 & -2.836029 \\
\hline 23 & 6 & 0 & -1.237903 & -1.758807 & 1.990496 \\
\hline 24 & 1 & 0 & -1.127620 & -1.595253 & 3.067950 \\
\hline 25 & 1 & 0 & -2.298332 & -1.721085 & 1.717240 \\
\hline 26 & 1 & 0 & -0.845586 & -2.746559 & 1.723934 \\
\hline 27 & 1 & 0 & -1.223570 & -1.758118 & -1.319701 \\
\hline 28 & 1 & 0 & -0.464409 & -0.653029 & -2.891320 \\
\hline
\end{tabular}

Basis set $A$ :

Selected geometrical parameters: H-H 2.021, Rh-H1 1.574, Rh-H2 1.565, Rh-S 2.419, Rh-P1 2.379, Rh-P4 2.394, S1-Rh-S2 172.2, P1-Rh-P4 101.7.

Frequencies:

\begin{tabular}{|c|c|c|c|c|}
\hline & & 1 & 2 & 3 \\
\hline & & A & A & A \\
\hline Frequencies & -- & 37.3958 & 45.3868 & 63.0713 \\
\hline Red. masses & -- & 4.2540 & 5.1961 & 4.5912 \\
\hline Frc consts & -- & 0.0035 & 0.0063 & 0.0108 \\
\hline IR Inten & -- & 2.5755 & 0.5532 & 2.2512 \\
\hline
\end{tabular}


$\left[\operatorname{Rh}\left(\mathrm{SPS}\left(\mathrm{PH}_{3}\right)(\mathrm{H})_{2}\right]\right.$ anti-PP (IIIa)

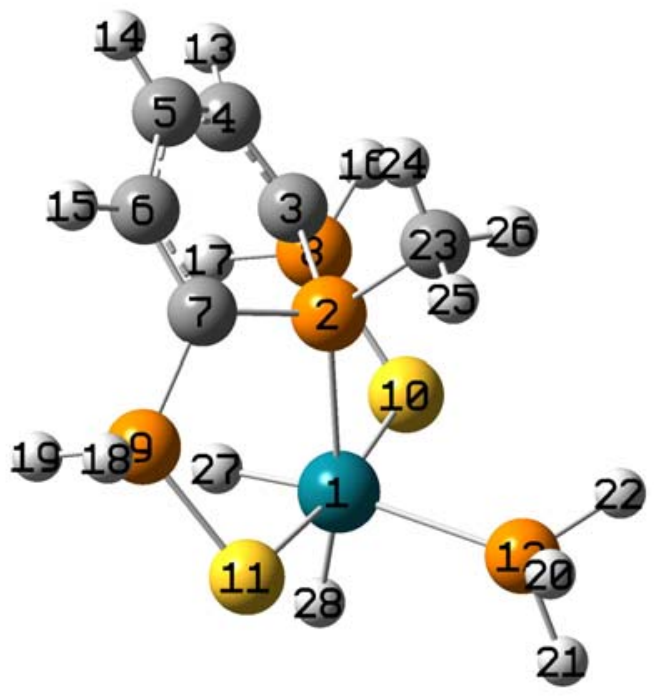

Optimized geometry:

\begin{tabular}{|c|c|c|c|c|c|}
\hline \multirow{2}{*}{$\begin{array}{l}\text { Center } \\
\text { Number }\end{array}$} & \multirow{2}{*}{$\begin{array}{l}\text { Atomic } \\
\text { Number }\end{array}$} & \multirow{2}{*}{$\begin{array}{c}\text { Atomic } \\
\text { Type }\end{array}$} & \multicolumn{3}{|c|}{ Coordinates (Angstroms) } \\
\hline & & & $\mathrm{X}$ & $\mathrm{Y}$ & Z \\
\hline & & & & & \\
\hline 1 & 45 & 0 & -0.158111 & -0.213854 & -1.303680 \\
\hline 2 & 15 & 0 & -0.151829 & -0.203276 & 1.075148 \\
\hline 3 & 6 & 0 & 1.558355 & -0.201964 & 1.633626 \\
\hline 4 & 6 & 0 & 1.884853 & 0.481609 & 2.803424 \\
\hline 5 & 6 & 0 & 1.020429 & 1.377970 & 3.458835 \\
\hline 6 & 6 & 0 & -0.086457 & 1.946212 & 2.801369 \\
\hline 7 & 6 & 0 & -0.645056 & 1.435016 & 1.631418 \\
\hline 8 & 15 & 0 & 2.717861 & -0.680507 & 0.390201 \\
\hline 9 & 15 & 0 & -1.435972 & 2.405628 & 0.385597 \\
\hline 10 & 16 & 0 & 1.873428 & -1.525552 & -1.252315 \\
\hline 11 & 16 & 0 & -2.002335 & 1.350539 & -1.254728 \\
\hline 12 & 15 & 0 & -1.556458 & -2.098711 & -1.778513 \\
\hline 13 & 1 & 0 & 2.899357 & 0.407686 & 3.203555 \\
\hline 14 & 1 & 0 & 1.349582 & 1.822285 & 4.393421 \\
\hline 15 & 1 & 0 & -0.450518 & 2.896697 & 3.199906 \\
\hline 16 & 1 & 0 & 3.687177 & -1.564259 & 0.919618 \\
\hline 17 & 1 & 0 & 3.550492 & 0.413240 & 0.039408 \\
\hline 18 & 1 & 0 & -2.560717 & 3.081557 & 0.913725 \\
\hline 19 & 1 & 0 & -0.627191 & 3.516275 & 0.032220 \\
\hline 20 & 1 & 0 & -2.905350 & -2.130748 & -1.343786 \\
\hline 21 & 1 & 0 & -1.816969 & -2.463356 & -3.120356 \\
\hline 22 & 1 & 0 & -1.205400 & -3.396887 & -1.330088 \\
\hline 23 & 6 & 0 & -1.002764 & -1.346911 & 2.247467 \\
\hline 24 & 1 & 0 & -0.797813 & -1.069451 & 3.286587 \\
\hline 25 & 1 & 0 & -2.082915 & -1.304346 & 2.067954 \\
\hline 26 & 1 & 0 & -0.649915 & -2.368980 & 2.069608 \\
\hline 27 & 1 & 0 & 0.774218 & 1.042640 & -1.252254 \\
\hline 28 & 1 & 0 & 0.033284 & 0.042715 & -2.844550 \\
\hline
\end{tabular}

Basis set $A$ :

Selected geometrical parameters: H-H 2.066, Rh-H1 1.603, Rh-H2 1.554, Rh-S 2.416, Rh-P1 2.330, Rh-P4 2.416, S1-Rh-S2 172.0, P1-Rh-P4 106.2.

Frequencies:

\begin{tabular}{|c|c|c|c|c|}
\hline & & 1 & 2 & 3 \\
\hline & & A & A & A \\
\hline Frequencies & -- & 36.9732 & 41.2622 & 62.8499 \\
\hline Red. masses & -- & 1.0771 & 4.0400 & 4.0281 \\
\hline Frc consts & -- & 0.0009 & 0.0041 & 0.0094 \\
\hline IR Inten & -- & 0.0865 & 1.0770 & 1.8883 \\
\hline
\end{tabular}


$\left[\operatorname{Rh}\left(\mathrm{SPS}\left(\mathrm{PH}_{3}\right)(\mathrm{H})_{2}\right]\right.$ anti-SS (IVa)

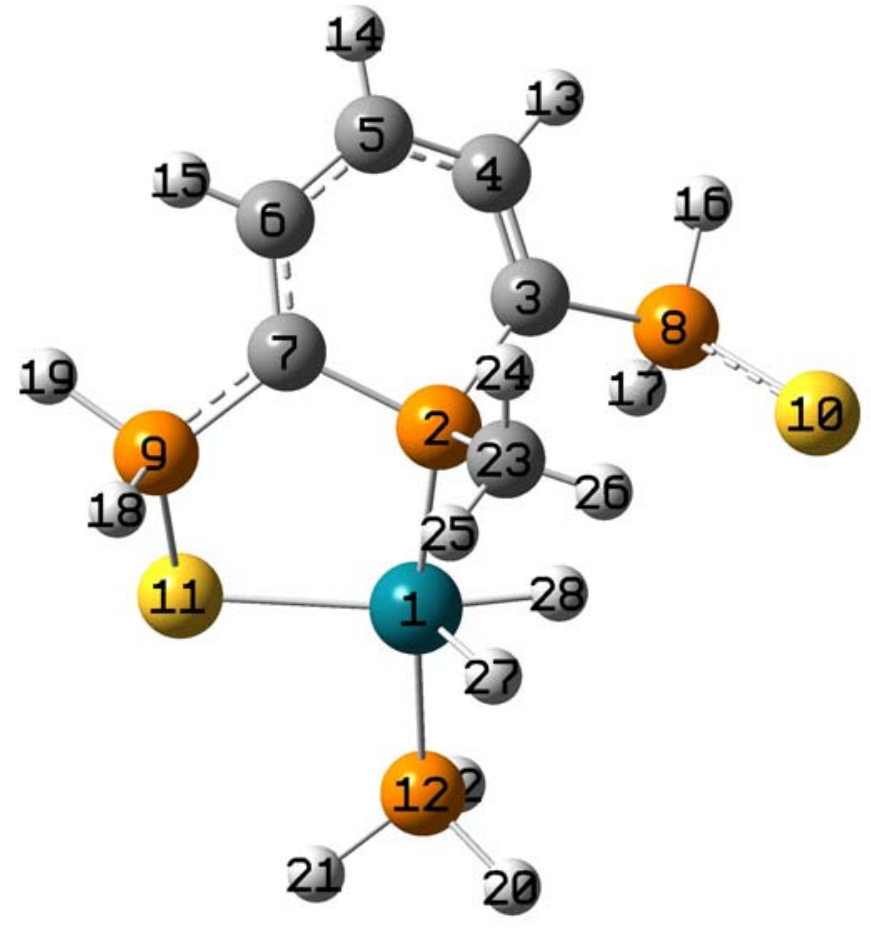

Optimized geometry:

\begin{tabular}{|c|c|c|c|c|c|}
\hline \multirow{2}{*}{$\begin{array}{l}\text { Center } \\
\text { Number }\end{array}$} & \multirow{2}{*}{$\begin{array}{l}\text { Atomic } \\
\text { Number }\end{array}$} & \multirow{2}{*}{$\begin{array}{l}\text { Atomic } \\
\text { Type }\end{array}$} & \multicolumn{3}{|c|}{ Coordinates (Angstroms) } \\
\hline & & & $\mathrm{X}$ & Y & Z \\
\hline 1 & 45 & 0 & -1.170634 & -0.834854 & 0.042763 \\
\hline 2 & 15 & 0 & 0.589138 & 0.576047 & 0.523002 \\
\hline 3 & 6 & 0 & 2.098557 & 0.438245 & -0.447224 \\
\hline 4 & 6 & 0 & 2.800777 & 1.575793 & -0.799548 \\
\hline 5 & 6 & 0 & 2.363815 & 2.911958 & -0.654275 \\
\hline 6 & 6 & 0 & 1.061595 & 3.216731 & -0.289577 \\
\hline 7 & 6 & 0 & 0.120337 & 2.269655 & 0.151305 \\
\hline 8 & 15 & 0 & 2.744515 & -1.176539 & -0.876554 \\
\hline 9 & 15 & 0 & -1.593778 & 2.583719 & 0.193454 \\
\hline 10 & 16 & 0 & 3.256638 & -2.444396 & 0.534200 \\
\hline 11 & 16 & 0 & -2.747421 & 1.023351 & -0.417017 \\
\hline 12 & 15 & 0 & -2.750712 & -2.496929 & -0.355586 \\
\hline 13 & 1 & 0 & 3.762268 & 1.435977 & -1.297980 \\
\hline 14 & 1 & 0 & 3.019730 & 3.710332 & -0.985510 \\
\hline 15 & 1 & 0 & 0.739057 & 4.252410 & -0.407025 \\
\hline 16 & 1 & 0 & 3.785773 & -0.808052 & -1.766169 \\
\hline 17 & 1 & 0 & 1.795046 & -1.703899 & -1.785656 \\
\hline 18 & 1 & 0 & -2.072412 & 2.984756 & 1.464569 \\
\hline 19 & 1 & 0 & -1.822493 & 3.770553 & -0.540035 \\
\hline 20 & 1 & 0 & -2.757785 & -3.610330 & 0.510610 \\
\hline 21 & 1 & 0 & -4.142322 & -2.242357 & -0.356529 \\
\hline 22 & 1 & 0 & -2.691064 & -3.207729 & -1.576813 \\
\hline 23 & 6 & 0 & 1.125480 & 0.491899 & 2.280720 \\
\hline 24 & 1 & 0 & 1.886637 & 1.254283 & 2.475903 \\
\hline 25 & 1 & 0 & 0.264092 & 0.648562 & 2.939413 \\
\hline 26 & 1 & 0 & 1.550503 & -0.500677 & 2.465323 \\
\hline 27 & 1 & 0 & -0.947132 & -1.452581 & 1.401947 \\
\hline 28 & 1 & 0 & -0.099657 & -1.946284 & -0.054463 \\
\hline
\end{tabular}

Basis set $A$ :

Selected geometrical parameters: H-H 1.756, Rh-H1 1.510, Rh-H2 1.547, Rh-S1 2.480, Rh-S2 4.736, Rh-P1 2.306, Rh-P4 2.328, S1-Rh-H2 124.6, S1-Rh-H1 165.3, P1-Rh-P4 172.1. Frequencies:

\begin{tabular}{|c|c|c|c|c|}
\hline & 1 & 2 & 3 \\
\hline & & A & A & A \\
\hline Frequencies & -- & 25.0674 & 41.1421 & 54.4054 \\
\hline Red. masses & -- & 10.0081 & 8.6362 & 1.3700 \\
\hline Frc consts & -- & 0.0037 & 0.0086 & 0.0024 \\
\hline IR Inten & -- & 1.1947 & 5.1285 & 0.1569 \\
\hline
\end{tabular}


$\left[\operatorname{Rh}\left(\mathrm{SPS}\left(\mathrm{PH}_{3}\right)(\mathrm{H})_{2}\right] \operatorname{trans}(\mathbf{V a})\right.$

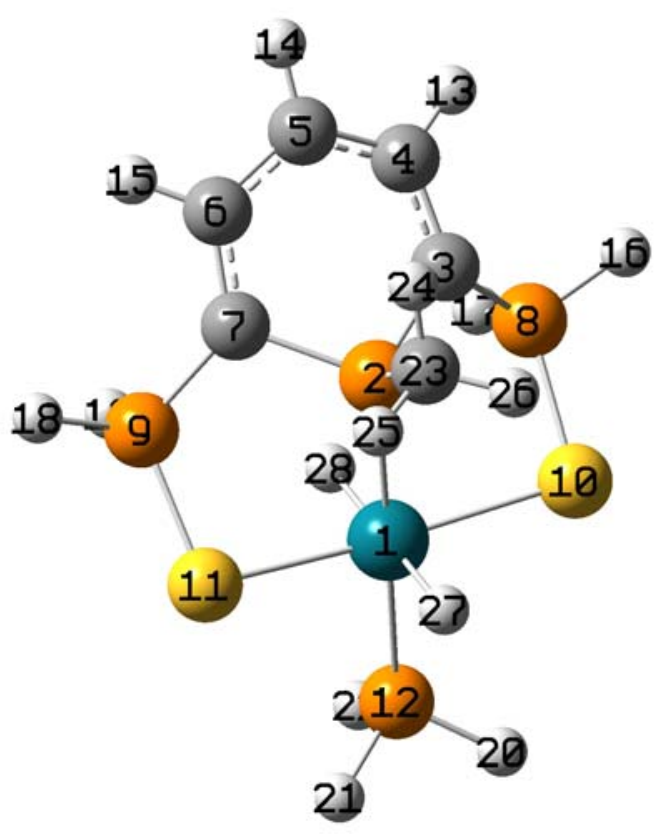

Optimized geometry:

\begin{tabular}{|c|c|c|c|c|c|}
\hline \multirow{2}{*}{$\begin{array}{l}\text { Center } \\
\text { Number }\end{array}$} & \multirow{2}{*}{$\begin{array}{l}\text { Atomic } \\
\text { Number }\end{array}$} & \multirow{2}{*}{$\begin{array}{l}\text { Atomic } \\
\text { Type }\end{array}$} & \multicolumn{3}{|c|}{ Coordinates (Angstroms) } \\
\hline & & & $\mathrm{x}$ & Y & Z \\
\hline 1 & 45 & 0 & -0.241955 & -0.345894 & -1.099368 \\
\hline 2 & 15 & 0 & -0.245055 & -0.350318 & 1.169306 \\
\hline 3 & 6 & 0 & 1.435340 & -0.343392 & 1.795979 \\
\hline 4 & 6 & 0 & 1.698585 & 0.285930 & 3.009865 \\
\hline 5 & 6 & 0 & 0.783739 & 1.120383 & 3.678832 \\
\hline 6 & 6 & 0 & -0.313812 & 1.693652 & 3.009866 \\
\hline 7 & 6 & 0 & -0.814722 & 1.230585 & 1.795979 \\
\hline 8 & 15 & 0 & 2.585793 & -0.738064 & 0.520856 \\
\hline 9 & 15 & 0 & -1.579942 & 2.175968 & 0.520858 \\
\hline 10 & 16 & 0 & 1.696979 & -1.798153 & -0.960789 \\
\hline 11 & 16 & 0 & -2.271005 & 0.977542 & -0.960783 \\
\hline 12 & 15 & 0 & -0.185046 & -0.264492 & -3.414117 \\
\hline 13 & 1 & 0 & 2.699983 & 0.213287 & 3.440717 \\
\hline 14 & 1 & 0 & 1.062434 & 1.518788 & 4.649601 \\
\hline 15 & 1 & 0 & -0.725412 & 2.609435 & 3.440720 \\
\hline 16 & 1 & 0 & 3.675984 & -1.458727 & 1.062074 \\
\hline 17 & 1 & 0 & 3.228577 & 0.418860 & 0.017242 \\
\hline 18 & 1 & 0 & -2.630728 & 2.952964 & 1.062074 \\
\hline 19 & 1 & 0 & -0.713558 & 3.176476 & 0.017240 \\
\hline 20 & 1 & 0 & 0.341083 & -1.371435 & -4.118542 \\
\hline 21 & 1 & 0 & -1.405303 & -0.149650 & -4.118484 \\
\hline 22 & 1 & 0 & 0.521867 & 0.745980 & -4.106988 \\
\hline 23 & 6 & 0 & -1.149885 & -1.643807 & 2.095274 \\
\hline 24 & 1 & 0 & -1.045866 & -1.495102 & 3.175308 \\
\hline 25 & 1 & 0 & -2.205796 & -1.598138 & 1.810040 \\
\hline 26 & 1 & 0 & -0.744932 & -2.620049 & 1.810045 \\
\hline 27 & 1 & 0 & -1.177977 & -1.683980 & -1.224268 \\
\hline 28 & 1 & 0 & 0.720341 & 1.029750 & -0.968372 \\
\hline
\end{tabular}

Basis set A:

Selected geometrical parameters: Rh-H1 1.638, Rh-H2 1.684, Rh-S1 2.426, Rh-S2 2.426, Rh-P1 2.269, Rh-P4 2.317, H1-Rh-H2 179.9, S1-Rh-S2 172.5, P1-Rh-P4 177.7.

Frequencies:

\begin{tabular}{|c|c|c|c|c|}
\hline & & 1 & 2 & 3 \\
\hline & & A & A & A \\
\hline Frequencies & -- & 32.9671 & 58.9814 & 68.6030 \\
\hline Red. masses & -- & 1.0581 & 5.8829 & 5.3254 \\
\hline Frc consts & -- & 0.0007 & 0.0121 & 0.0148 \\
\hline IR Inten & -- & 0.0119 & 1.3550 & 0.8061 \\
\hline
\end{tabular}


$\left[\operatorname{Rh}\left(\operatorname{SPS}\left(\mathrm{PH}_{3}\right)\left(\mathrm{H}_{2}\right)_{2}\right] \quad M H \ldots S H \quad(\mathrm{VIa})\right.$

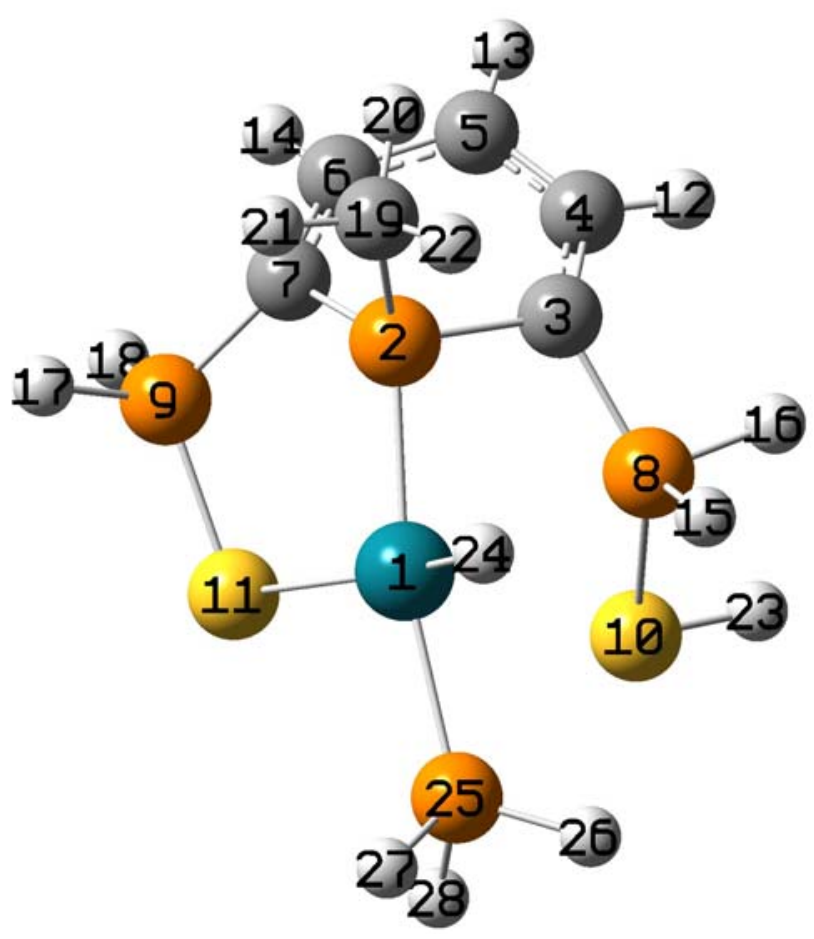

Optimized geometry:

\begin{tabular}{|c|c|c|c|c|c|}
\hline \multirow{2}{*}{$\begin{array}{l}\text { Center } \\
\text { Number }\end{array}$} & \multirow{2}{*}{$\begin{array}{l}\text { Atomic } \\
\text { Number }\end{array}$} & \multirow{2}{*}{$\begin{array}{l}\text { Atomic } \\
\text { Type }\end{array}$} & \multicolumn{3}{|c|}{ Coordinates (Angstroms) } \\
\hline & & & $\mathrm{x}$ & $\mathrm{Y}$ & Z \\
\hline 1 & 45 & 0 & 0.995558 & -0.436041 & 0.629807 \\
\hline 2 & 15 & 0 & -1.187148 & 0.013063 & 1.016800 \\
\hline 3 & 6 & 0 & -1.186002 & 1.669604 & 0.345125 \\
\hline 4 & 6 & 0 & -2.297701 & 2.161265 & -0.343987 \\
\hline 5 & 6 & 0 & -3.405271 & 1.371485 & -0.685539 \\
\hline 6 & 6 & 0 & -3.311388 & -0.035111 & -0.745599 \\
\hline 7 & 6 & 0 & -2.306436 & -0.793466 & -0.147341 \\
\hline 8 & 15 & 0 & 0.482641 & 2.145032 & 0.006835 \\
\hline 9 & 15 & 0 & -1.584513 & -2.223094 & -0.883483 \\
\hline 10 & 16 & 0 & 1.660930 & 1.702919 & -1.727385 \\
\hline 11 & 16 & 0 & 0.400383 & -2.034390 & -1.238567 \\
\hline 12 & 1 & 0 & -2.268305 & 3.181273 & -0.733398 \\
\hline 13 & 1 & 0 & -4.253060 & 1.846291 & -1.170646 \\
\hline 14 & 1 & 0 & -4.025912 & -0.533473 & -1.404221 \\
\hline 15 & 1 & 0 & 1.284807 & 2.526266 & 1.094576 \\
\hline 16 & 1 & 0 & 0.202454 & 3.485111 & -0.453894 \\
\hline 17 & 1 & 0 & -1.838649 & -3.382610 & -0.112558 \\
\hline 18 & 1 & 0 & -2.339755 & -2.519499 & -2.041984 \\
\hline 19 & 6 & 0 & -2.076555 & 0.062627 & 2.622947 \\
\hline 20 & 1 & 0 & -3.077054 & 0.488895 & 2.495841 \\
\hline 21 & 1 & 0 & -2.161245 & -0.951892 & 3.026598 \\
\hline 22 & 1 & 0 & -1.501182 & 0.676176 & 3.323690 \\
\hline 23 & 1 & 0 & 1.587090 & 2.940874 & -2.281545 \\
\hline 24 & 1 & 0 & 1.306172 & 0.293891 & 1.975693 \\
\hline 25 & 15 & 0 & 3.277692 & -0.483073 & 0.393419 \\
\hline 26 & 1 & 0 & 4.037440 & 0.678779 & 0.662805 \\
\hline 27 & 1 & 0 & 3.988387 & -1.354173 & 1.256885 \\
\hline 28 & 1 & 0 & 3.947721 & -0.847880 & -0.801126 \\
\hline
\end{tabular}

Basis set $A$ :

Selected geometrical parameters: Rh-H1 1.562, S2-H2 1.358, Rh-S1 2.530, Rh-S2 3.252, Rh-P1 2.262, Rh-P4 2.295, S1-Rh-H1 168.0, P1-Rh-P4 168.9.

Frequencies:

\begin{tabular}{|c|c|c|c|c|}
\hline & & 1 & 2 & 3 \\
\hline & & A & A & A \\
\hline Frequencies & -- & 45.1369 & 60.2594 & 69.3647 \\
\hline Red. masses & -- & 8.8494 & 9.7341 & 8.0839 \\
\hline Frc consts & -- & 0.0106 & 0.0208 & 0.0229 \\
\hline IR Inten & -- & 0.3648 & 5.3681 & 3.3635 \\
\hline
\end{tabular}


$\left[\operatorname{Rh}(\mathrm{SPS})\left(\mathrm{PH}_{3}\right)\left(\mathrm{H}_{2}\right)\right] \quad(\mathbf{T S}-\mathbf{I a})$

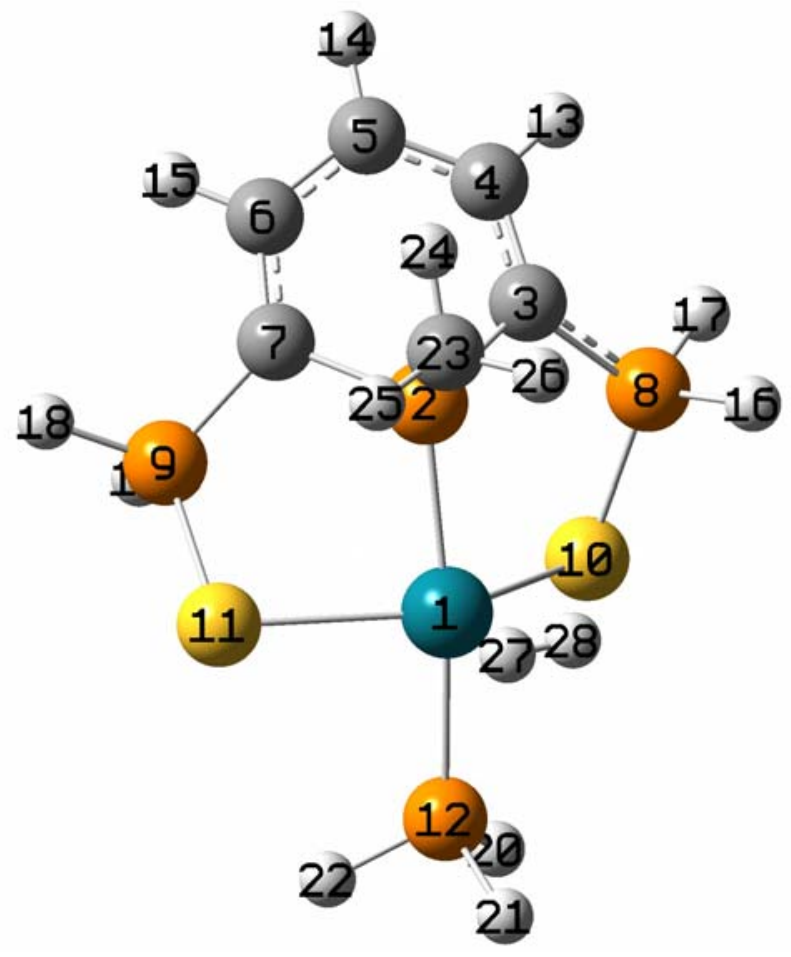

Optimized geometry:

\begin{tabular}{|c|c|c|c|c|c|}
\hline \multirow{2}{*}{$\begin{array}{l}\text { Center } \\
\text { Number }\end{array}$} & \multirow{2}{*}{$\begin{array}{l}\text { Atomic } \\
\text { Number }\end{array}$} & \multirow{2}{*}{$\begin{array}{l}\text { Atomic } \\
\text { Type }\end{array}$} & \multicolumn{3}{|c|}{ Coordinates (Angstroms) } \\
\hline & & & $\mathrm{X}$ & Y & Z \\
\hline 1 & 45 & 0 & -0.237550 & -0.471001 & -1.158214 \\
\hline 2 & 15 & 0 & -0.227467 & -0.511071 & 1.122180 \\
\hline 3 & 6 & 0 & 1.418711 & -0.493428 & 1.850419 \\
\hline 4 & 6 & 0 & 1.627651 & 0.111295 & 3.087160 \\
\hline 5 & 6 & 0 & 0.681594 & 0.913110 & 3.747531 \\
\hline 6 & 6 & 0 & -0.430418 & 1.443208 & 3.072550 \\
\hline 7 & 6 & 0 & -0.889595 & 0.999152 & 1.835619 \\
\hline 8 & 15 & 0 & 2.651521 & -0.877050 & 0.658909 \\
\hline 9 & 15 & 0 & -1.824247 & 1.928979 & 0.666447 \\
\hline 10 & 16 & 0 & 2.238908 & -0.066135 & -1.137459 \\
\hline 11 & 16 & 0 & -2.367004 & 0.794006 & -0.906499 \\
\hline 12 & 15 & 0 & -0.227311 & -0.057958 & -3.436611 \\
\hline 13 & 1 & 0 & 2.614611 & 0.038155 & 3.547986 \\
\hline 14 & 1 & 0 & 0.934707 & 1.314440 & 4.723838 \\
\hline 15 & 1 & 0 & -0.904246 & 2.317601 & 3.524554 \\
\hline 16 & 1 & 0 & 2.839122 & -2.278604 & 0.551393 \\
\hline 17 & 1 & 0 & 3.904488 & -0.487291 & 1.188832 \\
\hline 18 & 1 & 0 & -2.943863 & 2.499150 & 1.320912 \\
\hline 19 & 1 & 0 & -1.136321 & 3.107265 & 0.277180 \\
\hline 20 & 1 & 0 & 0.938789 & 0.456425 & -4.053682 \\
\hline 21 & 1 & 0 & -0.473907 & -1.085953 & -4.380879 \\
\hline 22 & 1 & 0 & -1.145426 & 0.885015 & -3.955640 \\
\hline 23 & 6 & 0 & -1.128200 & -1.883281 & 1.952800 \\
\hline 24 & 1 & 0 & -1.079068 & -1.766919 & 3.040708 \\
\hline 25 & 1 & 0 & -2.173851 & -1.864403 & 1.627645 \\
\hline 26 & 1 & 0 & -0.681352 & -2.841620 & 1.666170 \\
\hline 27 & 1 & 0 & -1.009191 & -2.174016 & -1.346395 \\
\hline 28 & 1 & 0 & -0.211100 & -2.353976 & -1.350043 \\
\hline
\end{tabular}

Basis set $A$ :

Selected geometrical parameters: H-H 0.818, Rh-H1 1.879, Rh-H2 1.893, S1-Rh 2.490, S2-Rh 2.509, P1-Rh 2.281, P4-Rh 2.316, S-Rh-S 131.6, P1-Rh-P4 170.7

$\mathrm{SCF}$ Done: $\mathrm{E}(\mathrm{RB}+\mathrm{HF}-\mathrm{PW} 91)=-2508.63557933$

Sum of electronic and zero-point Energies=

Sum of electronic and thermal Energies=

Sum of electronic and thermal Enthalpies=

Sum of electronic and thermal Free Energies=
A.U. after 9 cycles

$-2508.447932$

$-2508.429497$

$-2508.428552$

$-2508.493985$ 
Total

E (Thermal)
$\mathrm{KCal} / \mathrm{Mol}$

129.319

Frequencies:

$$
\begin{aligned}
& 1 \\
& \text { A }
\end{aligned}
$$

Frequencies - - $\quad-76.0214$

Red. masses -- 11.5838

Frc consts -- 0.0394

IR Inten -- 1.2815
CV

$\mathrm{Cal} / \mathrm{Mol}-\mathrm{Kelvin}$

69.036

Cal/Mol-Kelvin

137.715

50.5541

5.6161

0.0085

3.0124
3

0.8742

4.4266

0.0097

1.3516 
$\left[\operatorname{Ir}(\mathrm{SPS})\left(\mathrm{PH}_{3}\right)\right]$

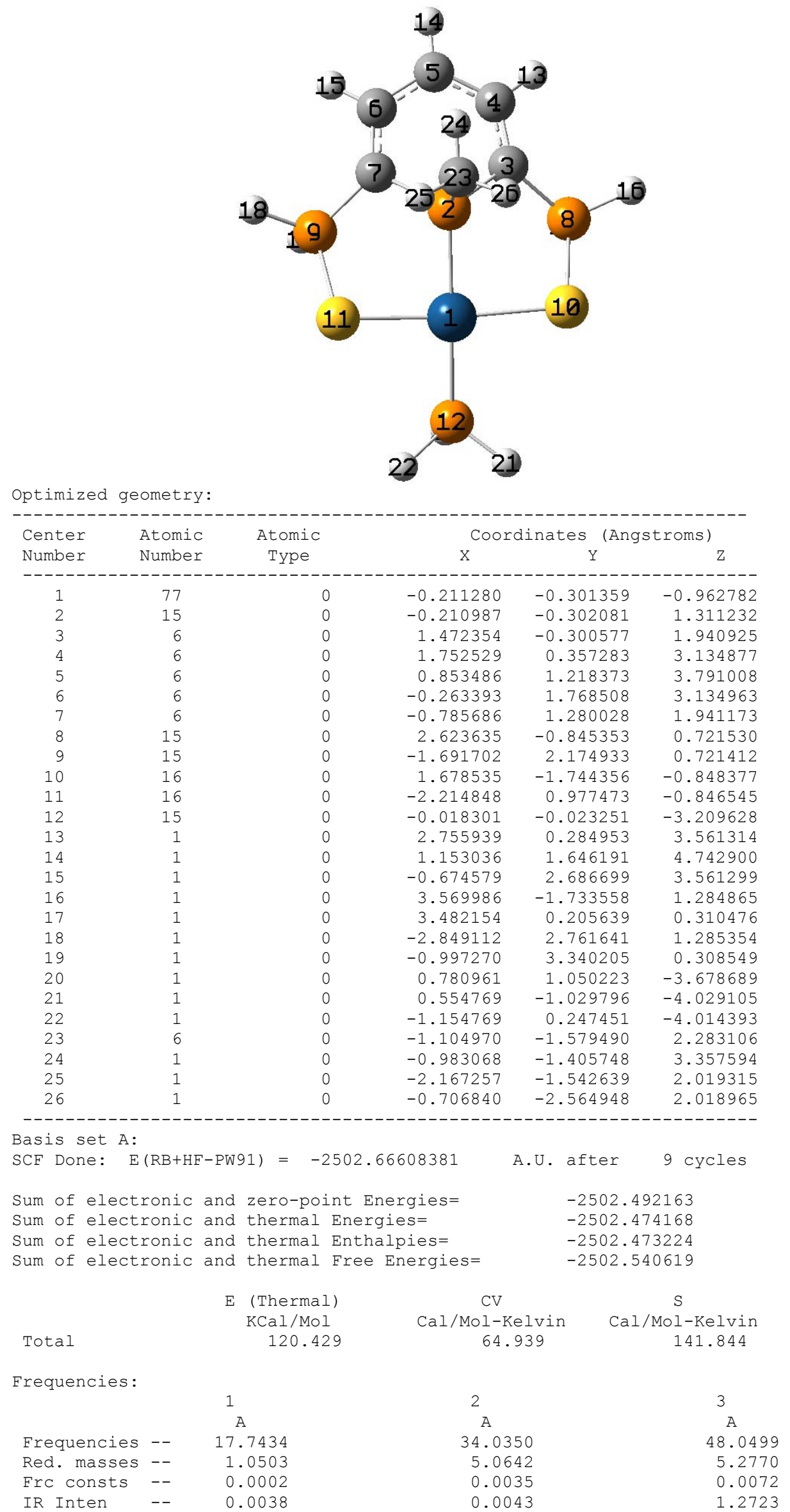


Complex [Ir(SPS) $\left.\left(\mathrm{PPh}_{3}\right)\right]$ : Optimized structure of real complex [Ir(SPS) ( $\left.\mathrm{PPh}_{3}\right)$ ] obtained at the ONIOM (B3PW91/UFF) level of theory. Atoms included in the QM part are shown in ball and stick format and atoms included in the MM part are represented by tubes. Hydrogen atoms have been omitted for clarity.

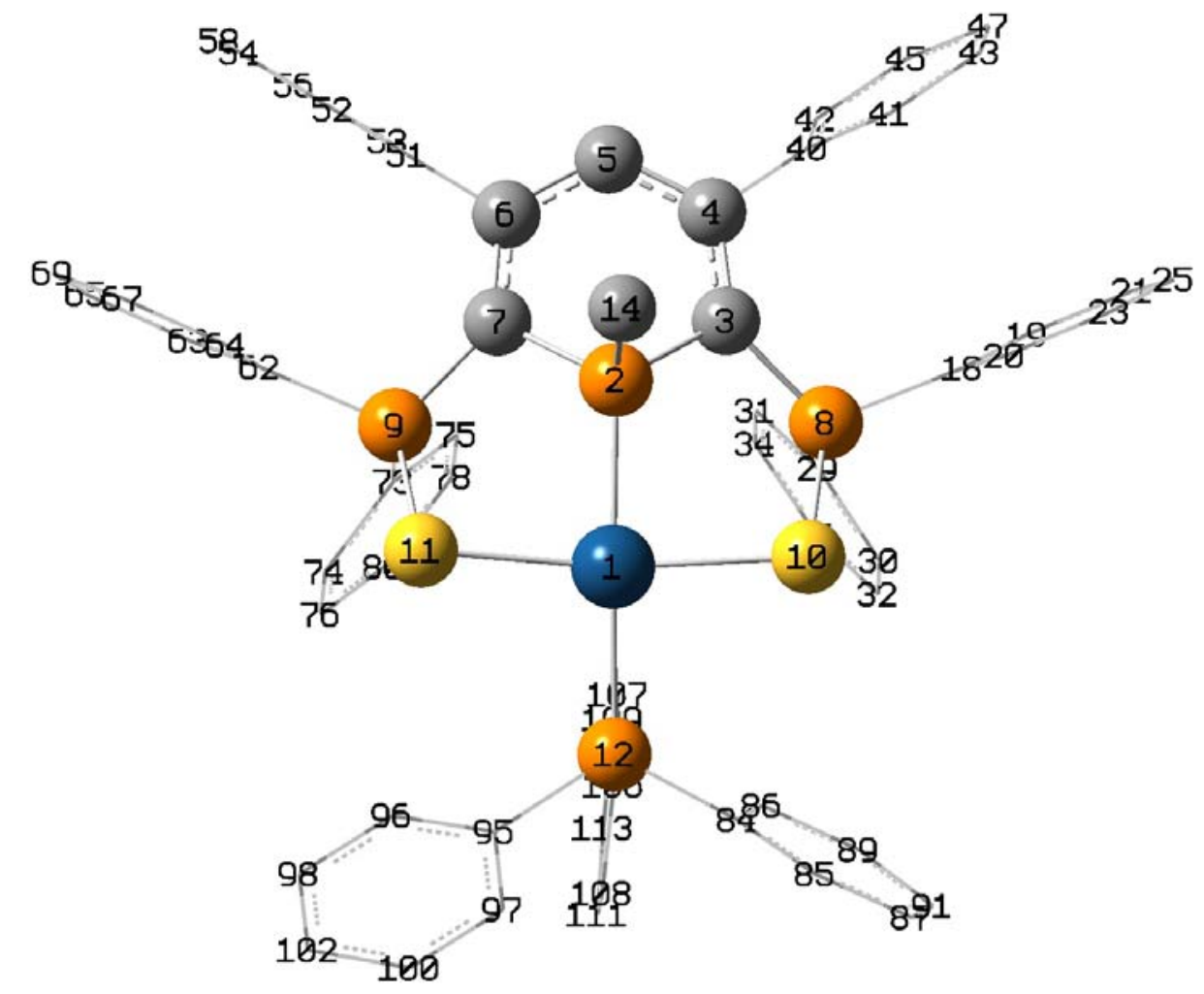

Optimized geometry:

\begin{tabular}{|c|c|c|c|c|c|}
\hline \multirow{2}{*}{$\begin{array}{l}\text { Center } \\
\text { Number }\end{array}$} & \multirow{2}{*}{$\begin{array}{l}\text { Atomic } \\
\text { Number }\end{array}$} & \multirow{2}{*}{$\begin{array}{c}\text { Atomic } \\
\text { Type }\end{array}$} & \multicolumn{3}{|c|}{ Coordinates (Angstroms) } \\
\hline & & & $\mathrm{X}$ & $\mathrm{Y}$ & Z \\
\hline 1 & 77 & 0 & 1.170666 & -0.151912 & -1.020345 \\
\hline 2 & 15 & 0 & -1.020178 & 0.113351 & -1.542889 \\
\hline 3 & 6 & 0 & -2.082081 & -1.114887 & -0.774065 \\
\hline 4 & 6 & 0 & -3.433766 & -0.772957 & -0.594151 \\
\hline 5 & 6 & 0 & -3.893947 & 0.555342 & -0.676007 \\
\hline 6 & 6 & 0 & -3.061702 & 1.698020 & -0.618128 \\
\hline 7 & 6 & 0 & -1.685499 & 1.632588 & -0.842419 \\
\hline 8 & 15 & 0 & -1.119848 & -2.477705 & -0.170062 \\
\hline 9 & 15 & 0 & -0.343123 & 2.698386 & -0.361341 \\
\hline 10 & 16 & 0 & 0.734798 & -2.482133 & -1.044050 \\
\hline 11 & 16 & 0 & 1.357681 & 2.182591 & -1.378069 \\
\hline 12 & 15 & 0 & 3.302753 & -0.467689 & -0.204000 \\
\hline 13 & 1 & 0 & -4.969064 & 0.718636 & -0.612389 \\
\hline 14 & 6 & 0 & -1.544107 & 0.149647 & -3.303886 \\
\hline 15 & 1 & 0 & -2.624943 & 0.304988 & -3.386030 \\
\hline 16 & 1 & 0 & -1.010990 & 0.962164 & -3.808562 \\
\hline 17 & 1 & 0 & -1.268067 & -0.802814 & -3.768506 \\
\hline 18 & 6 & 0 & -1.932894 & -4.038163 & -0.571612 \\
\hline 19 & 6 & 0 & -2.650375 & -4.756139 & 0.400663 \\
\hline 20 & 6 & 0 & -1.878979 & -4.525719 & -1.885866 \\
\hline 21 & 6 & 0 & -3.290122 & -5.952077 & 0.061761 \\
\hline 22 & 1 & 0 & -2.735391 & -4.389802 & 1.414404 \\
\hline 23 & 6 & 0 & -2.522748 & -5.720416 & -2.221455 \\
\hline 24 & 1 & 0 & -1.348775 & -3.976261 & -2.653321 \\
\hline 25 & 6 & 0 & -3.226077 & -6.434358 & -1.247911 \\
\hline 26 & 1 & 0 & -3.845480 & -6.499124 & 0.812475 \\
\hline 27 & 1 & 0 & -2.479963 & -6.090446 & -3.237605 \\
\hline 28 & 1 & 0 & -3.727023 & -7.357210 & -1.509779 \\
\hline 29 & 6 & 0 & -0.916527 & -2.458482 & 1.623843 \\
\hline 30 & 6 & 0 & -0.004921 & -3.338571 & 2.226278 \\
\hline
\end{tabular}




\begin{tabular}{|c|c|c|c|c|c|}
\hline 31 & 6 & 0 & -1.691647 & -1.611408 & 2.428666 \\
\hline 32 & 6 & 0 & 0.122167 & -3.376690 & 3.617760 \\
\hline 33 & 1 & 0 & 0.597600 & -4.003229 & 1.620292 \\
\hline 34 & 6 & 0 & -1.567142 & -1.655857 & 3.820118 \\
\hline 35 & 1 & 0 & -2.396706 & -0.923697 & 1.988513 \\
\hline 36 & 6 & 0 & -0.663399 & -2.540157 & 4.414938 \\
\hline 37 & 1 & 0 & 0.825986 & -4.057831 & 4.078039 \\
\hline 38 & 1 & 0 & -2.173357 & -1.006345 & 4.437731 \\
\hline 39 & 1 & 0 & -0.566317 & -2.571469 & 5.492291 \\
\hline 40 & 6 & 0 & -4.436065 & -1.821570 & -0.294355 \\
\hline 41 & 6 & 0 & -4.792126 & -2.752155 & -1.282659 \\
\hline 42 & 6 & 0 & -5.063941 & -1.869826 & 0.960137 \\
\hline 43 & 6 & 0 & -5.743319 & -3.739211 & -1.008282 \\
\hline 44 & 1 & 0 & -4.328449 & -2.715568 & -2.260872 \\
\hline 45 & 6 & 0 & -6.010190 & -2.862339 & 1.233033 \\
\hline 46 & 1 & 0 & -4.817690 & -1.144874 & 1.725645 \\
\hline 47 & 6 & 0 & -6.347261 & -3.798332 & 0.250835 \\
\hline 48 & 1 & 0 & -6.006533 & -4.462357 & -1.769183 \\
\hline 49 & 1 & 0 & -6.484279 & -2.904262 & 2.205022 \\
\hline 50 & 1 & 0 & -7.079018 & -4.566626 & 0.463991 \\
\hline 51 & 6 & 0 & -3.703078 & 2.984390 & -0.267531 \\
\hline 52 & 6 & 0 & -3.934367 & 3.946087 & -1.262572 \\
\hline 53 & 6 & 0 & -4.109648 & 3.238457 & 1.051095 \\
\hline 54 & 6 & 0 & -4.545238 & 5.160474 & -0.935967 \\
\hline 55 & 1 & 0 & -3.634529 & 3.757286 & -2.286120 \\
\hline 56 & 6 & 0 & -4.714992 & 4.456441 & 1.375153 \\
\hline 57 & 1 & 0 & -3.953929 & 2.498178 & 1.825612 \\
\hline 58 & 6 & 0 & -4.930710 & 5.417640 & 0.382969 \\
\hline 59 & 1 & 0 & -4.712415 & 5.904916 & -1.703422 \\
\hline 60 & 1 & 0 & -5.018559 & 4.654540 & 2.394887 \\
\hline 61 & 1 & 0 & -5.397813 & 6.360573 & 0.635672 \\
\hline 62 & 6 & 0 & -0.756400 & 4.411303 & -0.753506 \\
\hline 63 & 6 & 0 & -1.173328 & 5.306135 & 0.246694 \\
\hline 64 & 6 & 0 & -0.694330 & 4.848161 & -2.084973 \\
\hline 65 & 6 & 0 & -1.507272 & 6.623032 & -0.082909 \\
\hline 66 & 1 & 0 & -1.260367 & 4.989154 & 1.276777 \\
\hline 67 & 6 & 0 & -1.032515 & 6.164886 & -2.411117 \\
\hline 68 & 1 & 0 & -0.394813 & 4.167695 & -2.871895 \\
\hline 69 & 6 & 0 & -1.436627 & 7.052517 & -1.410541 \\
\hline 70 & 1 & 0 & -1.832494 & 7.307408 & 0.689833 \\
\hline 71 & 1 & 0 & -0.985339 & 6.495919 & -3.440375 \\
\hline 72 & 1 & 0 & -1.701672 & 8.070538 & -1.664753 \\
\hline 73 & 6 & 0 & -0.013817 & 2.629848 & 1.410661 \\
\hline 74 & 6 & 0 & 1.095789 & 3.307708 & 1.935760 \\
\hline 75 & 6 & 0 & -0.873561 & 1.938995 & 2.276456 \\
\hline 76 & 6 & 0 & 1.345411 & 3.290543 & 3.310822 \\
\hline 77 & 1 & 0 & 1.757714 & 3.861494 & 1.282422 \\
\hline 78 & 6 & 0 & -0.629991 & 1.935114 & 3.652778 \\
\hline 79 & 1 & 0 & -1.733636 & 1.410464 & 1.894766 \\
\hline 80 & 6 & 0 & 0.479763 & 2.608850 & 4.169952 \\
\hline 81 & 1 & 0 & 2.204577 & 3.812872 & 3.711000 \\
\hline 82 & 1 & 0 & -1.299273 & 1.407213 & 4.318804 \\
\hline 83 & 1 & 0 & 0.670960 & 2.598703 & 5.235061 \\
\hline 84 & 6 & 0 & 4.022833 & -2.056408 & -0.758331 \\
\hline 85 & 6 & 0 & 4.378435 & -3.078262 & 0.133454 \\
\hline 86 & 6 & 0 & 4.103595 & -2.332840 & -2.208348 \\
\hline 87 & 6 & 0 & 4.851971 & -4.302580 & -0.348576 \\
\hline 88 & 1 & 0 & 4.278178 & -2.952810 & 1.200409 \\
\hline 89 & 6 & 0 & 4.580356 & -3.566298 & -2.648045 \\
\hline 90 & 1 & 0 & 3.795266 & -1.584516 & -2.927245 \\
\hline 91 & 6 & 0 & 4.957295 & -4.542147 & -1.722716 \\
\hline 92 & 1 & 0 & 5.129903 & -5.080201 & 0.351184 \\
\hline 93 & 1 & 0 & 4.651785 & -3.770667 & -3.708409 \\
\hline 94 & 1 & 0 & 5.322929 & -5.498849 & -2.072819 \\
\hline 95 & 6 & 0 & 4.526891 & 0.845665 & -0.568116 \\
\hline 96 & 6 & 0 & 4.371335 & 2.103824 & 0.033495 \\
\hline 97 & 6 & 0 & 5.637871 & 0.619038 & -1.398662 \\
\hline 98 & 6 & 0 & 5.290611 & 3.127085 & -0.211726 \\
\hline 99 & 1 & 0 & 3.542398 & 2.287817 & 0.702449 \\
\hline 100 & 6 & 0 & 6.556998 & 1.644748 & -1.642242 \\
\hline 101 & 1 & 0 & 5.809126 & -0.341193 & -1.858016 \\
\hline 102 & 6 & 0 & 6.381751 & 2.898862 & -1.052618 \\
\hline 103 & 1 & 0 & 5.159202 & 4.094956 & 0.254428 \\
\hline 104 & 1 & 0 & 7.408381 & 1.465491 & -2.286261 \\
\hline 105 & 1 & 0 & 7.094889 & 3.690722 & -1.241600 \\
\hline 106 & 6 & 0 & 3.309149 & -0.506323 & 1.614188 \\
\hline 107 & 6 & 0 & 2.035958 & -0.411577 & 2.349434 \\
\hline
\end{tabular}




$\begin{array}{llllll}108 & 6 & 0 & 4.506901 & -0.540027 & 2.337138 \\ 109 & 6 & 0 & 2.056885 & -0.389840 & 3.743314 \\ 110 & 1 & 0 & 1.096706 & -0.350258 & 1.816040 \\ 111 & 6 & 0 & 4.484629 & -0.517077 & 3.734545 \\ 112 & 1 & 0 & 5.456780 & -0.586305 & 1.819704 \\ 113 & 6 & 0 & 3.272456 & -0.445023 & 4.430137 \\ 114 & 1 & 0 & 1.131102 & -0.321154 & 4.296797 \\ 115 & 1 & 0 & 5.416006 & -0.550491 & 4.284793 \\ 116 & 1 & 0 & 3.276342 & -0.424706 & 5.512223\end{array}$

Basis set $A$ :

Frequencies:

1

2

3

$\begin{array}{lr}\text { Frequencies -- } & 11.2725 \\ \text { Red. masses -- } & 6.1654\end{array}$

16.5346

5.8195

0.0573

Frc consts -- $\quad .0005$

.0009

.0541

5.7872

.0014

.0295

Basis set $B$ :

$\mathrm{SCF}$ Done: $\mathrm{E}(\mathrm{RB}+\mathrm{HF}-\mathrm{PW} 91)=-4580.93771475$

A.U. after 24 cycles 


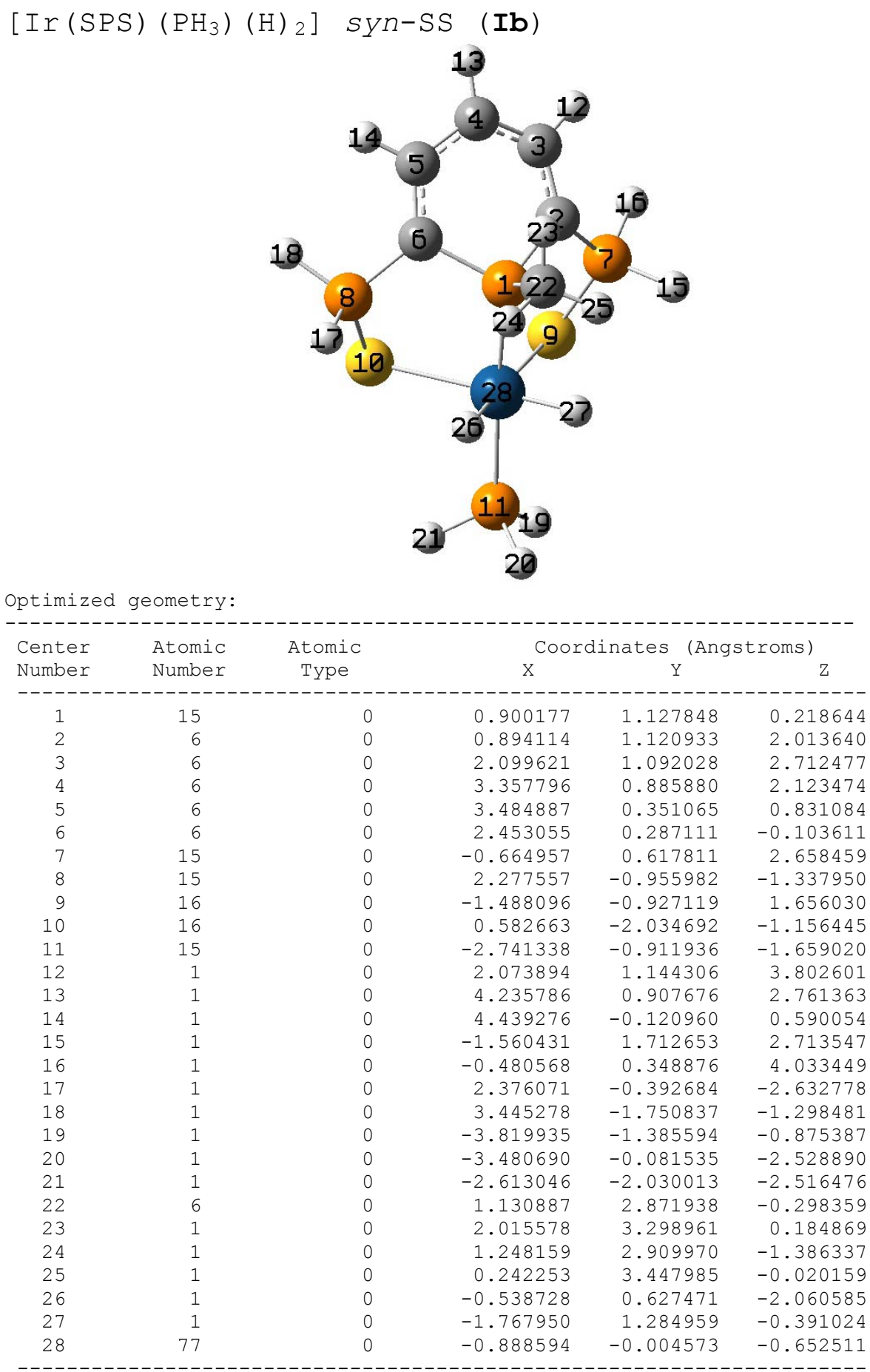

Basis set $A$ :

Selected geometrical parameters : H-H 2.175, Ir-H 1.583, Ir-S 2.557, Ir-P1 2.289, Ir-P4 2.295, S1-Ir-S2 91.5, P1-Ir-P4 171.2.

$\mathrm{SCF}$ Done: $\mathrm{E}(\mathrm{RB}+\mathrm{HF}-\mathrm{PW} 91)=-2503.90134550$

Sum of electronic and zero-point Energies=

Sum of electronic and thermal Energies=

Sum of electronic and thermal Enthalpies=

Sum of electronic and thermal Free Energies=

\section{A.U. after \\ 9 cycles}

$-2503.708642$

$-2503.690583$

$-2503.689639$

$-2503.755113$

$$
\begin{gathered}
\text { E (Thermal) } \\
\text { KCal/Mol }
\end{gathered}
$$$$
132.256
$$

CV

$\mathrm{Cal} / \mathrm{Mol}-\mathrm{Kelvin}$ 67.172
$\mathrm{Cal} / \mathrm{Mol}-\mathrm{Kelvin}$ 137.803

Frequencies: 
Frequencies -- $\quad 53.1868$

Red. masses -- 6.2721

Frc consts -- 0.0105

67.6716

4.8313

70.6646

0.0130

1.0927

0.0032

IR Inten -- $\quad 0.3425$

0.6012

0.1154 
$\left[\operatorname{Ir}(\mathrm{SPS})\left(\mathrm{PPh}_{3}\right)\left(\mathrm{H}_{2}\right)_{2}\right]$ syn-SS (Ia-rc) : Optimized structure of real complex [Ir(SPS) $\left(\mathrm{PPh}_{3}\right)$ ] obtained at the ONIOM (B3PW91/UFF) level of theory. Atoms included in the $Q M$ part are shown in ball and stick format and atoms included in the MM part are represented by tubes.

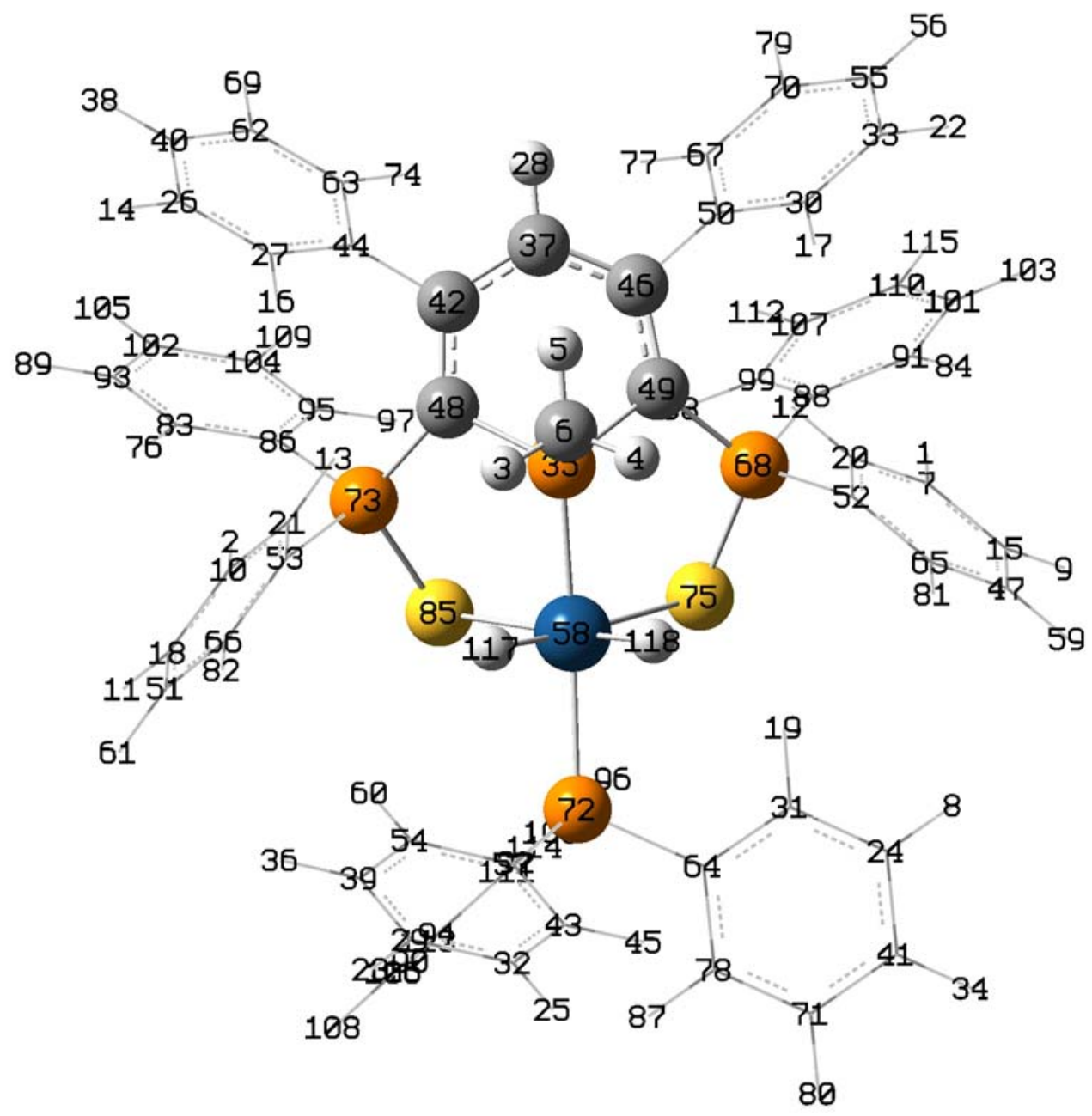

Optimized geometry:

\begin{tabular}{|c|c|c|c|c|c|}
\hline \multirow{2}{*}{$\begin{array}{l}\text { Center } \\
\text { Number }\end{array}$} & \multirow{2}{*}{$\begin{array}{l}\text { Atomic } \\
\text { Number }\end{array}$} & \multirow{2}{*}{$\begin{array}{c}\text { Atomic } \\
\text { Type }\end{array}$} & \multicolumn{3}{|c|}{ Coordinates (Angstroms) } \\
\hline & & & $\mathrm{X}$ & Y & Z \\
\hline 1 & 1 & 0 & -0.625691 & -4.672744 & -4.262739 \\
\hline 2 & 1 & 0 & 1.298908 & 4.373989 & -4.128097 \\
\hline 3 & 1 & 0 & -0.133587 & 0.859035 & -3.486509 \\
\hline 4 & 1 & 0 & -0.419306 & -0.884596 & -3.484003 \\
\hline 5 & 1 & 0 & -1.814737 & 0.239848 & -3.473145 \\
\hline 6 & 6 & 0 & -0.795020 & 0.072798 & -3.112383 \\
\hline 7 & 6 & 0 & -0.465405 & -4.740223 & -3.194468 \\
\hline 8 & 1 & 0 & 3.158423 & -5.435182 & -0.192341 \\
\hline 9 & 1 & 0 & 0.556210 & -6.632278 & -3.308883 \\
\hline 10 & 6 & 0 & 1.423106 & 4.363432 & -3.052984 \\
\hline 11 & 1 & 0 & 3.143148 & 5.658513 & -3.081635 \\
\hline 12 & 1 & 0 & -1.438605 & -2.878751 & -2.789301 \\
\hline 13 & 1 & 0 & -0.252520 & 3.072278 & -2.730609 \\
\hline 14 & 1 & 0 & -3.524330 & 6.168386 & -2.215428 \\
\hline 15 & 6 & 0 & 0.200800 & -5.844663 & -2.657221 \\
\hline 16 & 1 & 0 & -2.399078 & 3.982520 & -2.400453 \\
\hline 17 & 1 & 0 & -3.745926 & -3.008830 & -2.405177 \\
\hline 18 & 6 & 0 & 2.461925 & 5.088390 & -2.463445 \\
\hline 19 & 1 & 0 & 2.346841 & -3.120846 & -0.415798 \\
\hline 20 & 6 & 0 & -0.925550 & -3.722178 & -2.354172 \\
\hline 21 & 6 & 0 & 0.544354 & 3.623583 & -2.256279 \\
\hline 22 & 1 & 0 & -5.567347 & -4.651530 & -2.156469 \\
\hline
\end{tabular}




\begin{tabular}{|c|c|c|}
\hline 6.746165 & 2.159630 & -3.609563 \\
\hline 3.781872 & -4.612380 & 0.130907 \\
\hline 7.043841 & -0.273758 & -3.244877 \\
\hline 3.837783 & 5.346684 & -1.584737 \\
\hline 3.196693 & 4.108071 & -1.682375 \\
\hline 4766291 & 0.777460 & -1.426844 \\
\hline .148832 & 1.646620 & -2.867036 \\
\hline .497131 & -2.854600 & -1.644419 \\
\hline 323786 & -3.297110 & 0.006954 \\
\hline .318485 & 0.275410 & -2.658582 \\
\hline .5 .5339 & -3.783174 & -1.511497 \\
\hline .3974 & -5.885491 & 0.7 \\
\hline-0.7505 & 0.068524 & -1.275689 \\
\hline 5.0647 & 3.413951 & -2.288892 \\
\hline 年 & 0.594890 & -1.1 \\
\hline & 6.487131 & -0 . \\
\hline .2015 & 2.353342 & -2.123055 \\
\hline • 0 & 5.525989 & -0.671508 \\
\hline & -4.866734 & 0.672001 \\
\hline-2 . & 1.724127 & -0.956725 \\
\hline 5.5455 & -0.391128 & -1.702177 \\
\hline & 3.039104 & -0.861899 \\
\hline$r$ & -1.456020 & -1.58 \\
\hline-3.3418 & -0.741608 & -0.940001 \\
\hline 0.407616 & -5.931939 & -1.278385 \\
\hline-1.5161 & 1.6225 & -0.7 \\
\hline 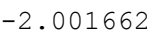 & -1.128504 & -0.774066 \\
\hline P & -1.729253 & -0.806310 \\
\hline - & 5.075926 & -1.07 \\
\hline 10 & -3.803371 & -0.96 \\
\hline .70256 & 3.601768 & -0.863046 \\
\hline & 1.690341 & -1.16 \\
\hline 6 & -3.592271 & -0.5 \\
\hline .32479 & -4.314776 & -0.440883 \\
\hline 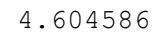 & 0.317382 & -0.93 \\
\hline 1.27 & -0.261516 & -0.3 \\
\hline 0.923574 & -6.787326 & -0.861936 \\
\hline 192 & 2.247076 & -0 . \\
\hline 671 & 5.635575 & -0.6 \\
\hline 8 & 4.464446 & 0.1 \\
\hline .65 & 3.223173 & 0.0 \\
\hline 58 & -2.22 & 0 \\
\hline 52 & -4.913752 & -0.43 \\
\hline L. 745744 & 4.335133 & -0.277077 \\
\hline 633 & -1.53 & 0.1 \\
\hline 16 & -2.49 & 0.1 \\
\hline 094859 & 4.604616 & 0.84 \\
\hline 41 & -2.467976 & 0.2 \\
\hline 66 & -3.80 & 1.0 \\
\hline .528868 & -0.511574 & 0.285811 \\
\hline 397726 & 2.62 & 0.1 \\
\hline 40 & 2.412159 & 391 \\
\hline 262567 & -1.949 & 1.3 \\
\hline 870193 & 5.555378 & 0.037738 \\
\hline & -0.67 & 791 \\
\hline 397282 & -2.489457 & 0.965709 \\
\hline .255731 & -2.321221 & 1.030800 \\
\hline 37095 & -4.002318 & 1.506771 \\
\hline 120 & -4.991969 & 0.630168 \\
\hline .882590 & 4.330334 & 0.796873 \\
\hline 405 & 5.152110 & 0.886019 \\
\hline$=0$ & -5.020462 & 0.058742 \\
\hline .750578 & 1.524933 & 1.424543 \\
\hline 98 & 3.780945 & 1.189402 \\
\hline 033 & -1.676058 & 1.289840 \\
\hline 562736 & -3.202618 & 1.207648 \\
\hline 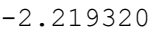 & 7.071407 & 1.424198 \\
\hline 563023 & 1.483907 & 1.253311 \\
\hline 14959 & -4.448709 & 0.928804 \\
\hline 946 & 0.193781 & 1.920857 \\
\hline 177091 & 6.017606 & 1.666939 \\
\hline 969338 & 1.143889 & 2.087761 \\
\hline 88 & 3.291962 & 2.281560 \\
\hline 468291 & -1.003497 & 2.945829 \\
\hline ח & 2.236215 & 2.521930 \\
\hline & -1.508002 & 2.551196 \\
\hline & -2.47661 & \\
\hline
\end{tabular}




\begin{tabular}{rrrrrr}
100 & 6 & 0 & 3.241238 & -0.254973 & 3.047468 \\
101 & 6 & 0 & -4.148297 & -4.958083 & 1.764131 \\
102 & 6 & 0 & -2.904230 & 5.522599 & 2.752348 \\
103 & 1 & 0 & -4.603700 & -5.914064 & 1.540554 \\
104 & 6 & 0 & -2.860083 & 4.160423 & 3.059376 \\
105 & 1 & 0 & -3.505746 & 6.193402 & 3.351839 \\
106 & 6 & 0 & 5.254683 & 1.656022 & 3.357271 \\
107 & 6 & 0 & -3.991597 & -2.988694 & 3.160961 \\
108 & 1 & 0 & 6.041501 & 2.389157 & 3.479628 \\
109 & 1 & 0 & -3.428283 & 3.776211 & 3.896418 \\
110 & 6 & 0 & -4.569246 & -4.229055 & 2.879074 \\
111 & 6 & 0 & 3.528039 & 0.259678 & 4.314747 \\
112 & 1 & 0 & -4.322359 & -2.421076 & 4.020965 \\
113 & 6 & 0 & 4.531660 & 1.218233 & 4.469274 \\
114 & 1 & 0 & 2.973588 & -0.086956 & 5.177195 \\
115 & 1 & 0 & -5.346784 & -4.622463 & 3.520825 \\
116 & 1 & 0 & 4.754378 & 1.615584 & 5.450994 \\
\hline
\end{tabular}

Basis set $A$ :

Frequencies:

1

2

3

4.1525

15.5418

5.8165

.0008

.0095

19.7962

6.0254

.0014

Frc consts -- $\quad .0007$

IR Inten -- .0219

Basis set $B$ :

$\mathrm{SCF}$ Done: $\mathrm{E}(\mathrm{RB}+\mathrm{HF}-\mathrm{PW} 91)=-4582.19076218$

A.U. after 32 cycles 
$\left[\operatorname{Ir}(\mathrm{SPS})\left(\mathrm{PH}_{3}\right)(\mathrm{H})_{2}\right] \quad$ syn-PP $(\mathbf{I I b})$

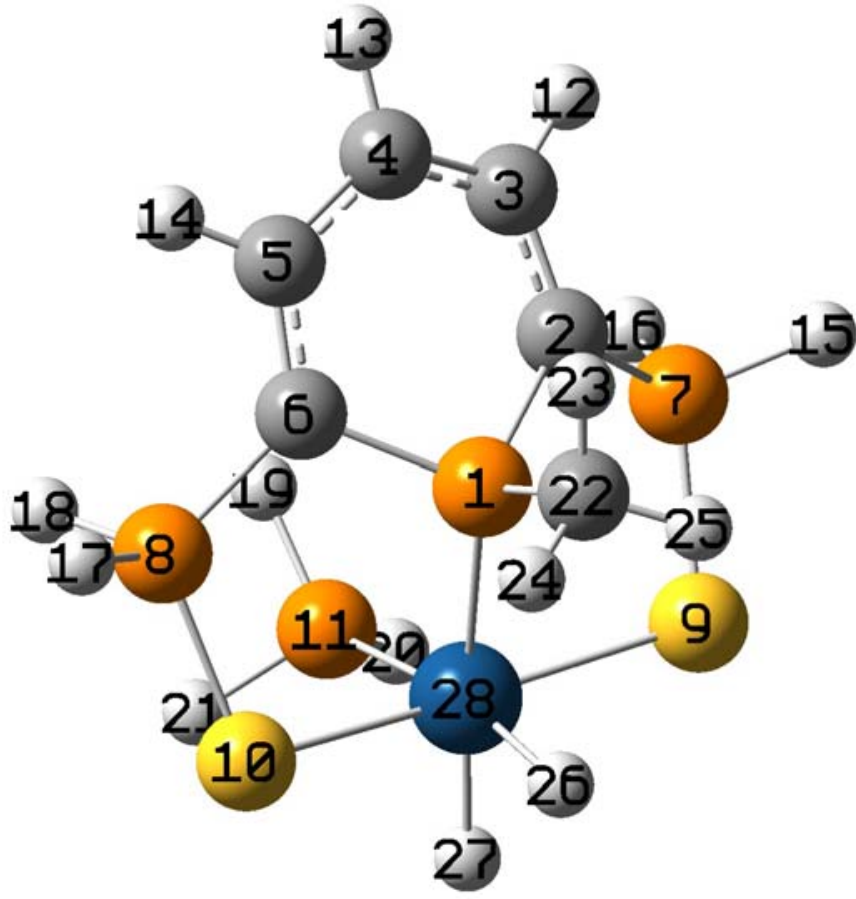

Optimized geometry:

\begin{tabular}{|c|c|c|c|c|c|}
\hline \multirow{2}{*}{$\begin{array}{l}\text { Center } \\
\text { Number }\end{array}$} & \multirow{2}{*}{$\begin{array}{l}\text { Atomic } \\
\text { Number }\end{array}$} & \multirow{2}{*}{$\begin{array}{l}\text { Atomic } \\
\text { Type }\end{array}$} & \multicolumn{3}{|c|}{ Coordinates (Angstroms) } \\
\hline & & & $\mathrm{x}$ & Y & Z \\
\hline 1 & 15 & 0 & 0.665633 & 1.087880 & 0.177531 \\
\hline 2 & 6 & 0 & 0.665107 & 1.099374 & 1.976376 \\
\hline 3 & 6 & 0 & 1.869492 & 1.183784 & 2.669149 \\
\hline 4 & 6 & 0 & 3.139234 & 1.083675 & 2.071824 \\
\hline 5 & 6 & 0 & 3.327890 & 0.602340 & 0.763460 \\
\hline 6 & 6 & 0 & 2.308750 & 0.444107 & -0.171358 \\
\hline 7 & 15 & 0 & -0.920059 & 0.700549 & 2.641201 \\
\hline 8 & 15 & 0 & 2.324735 & -0.593217 & -1.598792 \\
\hline 9 & 16 & 0 & -2.350352 & 0.419765 & 1.194286 \\
\hline 10 & 16 & 0 & 0.495234 & -0.714294 & -2.524159 \\
\hline 11 & 15 & 0 & -0.589342 & -2.418022 & 0.286701 \\
\hline 12 & 1 & 0 & 1.854350 & 1.259247 & 3.758853 \\
\hline 13 & 1 & 0 & 4.013138 & 1.199571 & 2.705252 \\
\hline 14 & 1 & 0 & 4.337812 & 0.269075 & 0.513715 \\
\hline 15 & 1 & 0 & -1.396219 & 1.691062 & 3.530503 \\
\hline 16 & 1 & 0 & -0.807607 & -0.387864 & 3.549352 \\
\hline 17 & 1 & 0 & 3.267507 & -0.168638 & -2.562954 \\
\hline 18 & 1 & 0 & 2.883419 & -1.859925 & -1.274210 \\
\hline 19 & 1 & 0 & 0.439193 & -2.809933 & 1.195467 \\
\hline 20 & 1 & 0 & -1.657784 & -2.984102 & 1.020997 \\
\hline 21 & 1 & 0 & -0.397056 & -3.485087 & -0.621955 \\
\hline 22 & 6 & 0 & 0.711958 & 2.853912 & -0.325872 \\
\hline 23 & 1 & 0 & 1.545672 & 3.379594 & 0.151759 \\
\hline 24 & 1 & 0 & 0.814830 & 2.907326 & -1.415380 \\
\hline 25 & 1 & 0 & -0.235420 & 3.326078 & -0.043035 \\
\hline 26 & 1 & 0 & -1.554686 & 0.948713 & -1.479154 \\
\hline 27 & 1 & 0 & -2.219675 & -1.092667 & -1.365444 \\
\hline 28 & 77 & 0 & -0.981229 & -0.295338 & -0.660868 \\
\hline
\end{tabular}

Basis set A

Selected geometrical parameters : H-H 2.104, Ir-H1 1.611, Ir-H2 1.607, Ir-S1 2.416, Ir-S2 2.418, Ir-P1 2.345, Ir-P4 2.343, S1-Ir-S2 173.0, P1-Ir-H1 168.9, P4-Ir-H2 171.6. Frequencies:

\begin{tabular}{|c|c|c|c|c|}
\hline \multirow[t]{2}{*}{ 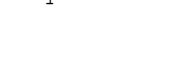 } & & 1 & 2 & 3 \\
\hline & & A & A & A \\
\hline Frequencies & -- & 36.5757 & 46.4519 & 76.8009 \\
\hline Red. masses & -- & 4.0766 & 5.3277 & 4.6568 \\
\hline Frc consts & -- & 0.0032 & 0.0068 & 0.0162 \\
\hline IR Inten & -- & 3.3162 & 0.2459 & 1.4541 \\
\hline
\end{tabular}


$\left[\mathrm{Rh}\left(\mathrm{SPS}\left(\mathrm{PH}_{3}\right)(\mathrm{H})_{2}\right]\right.$ anti-PP (IIIb)

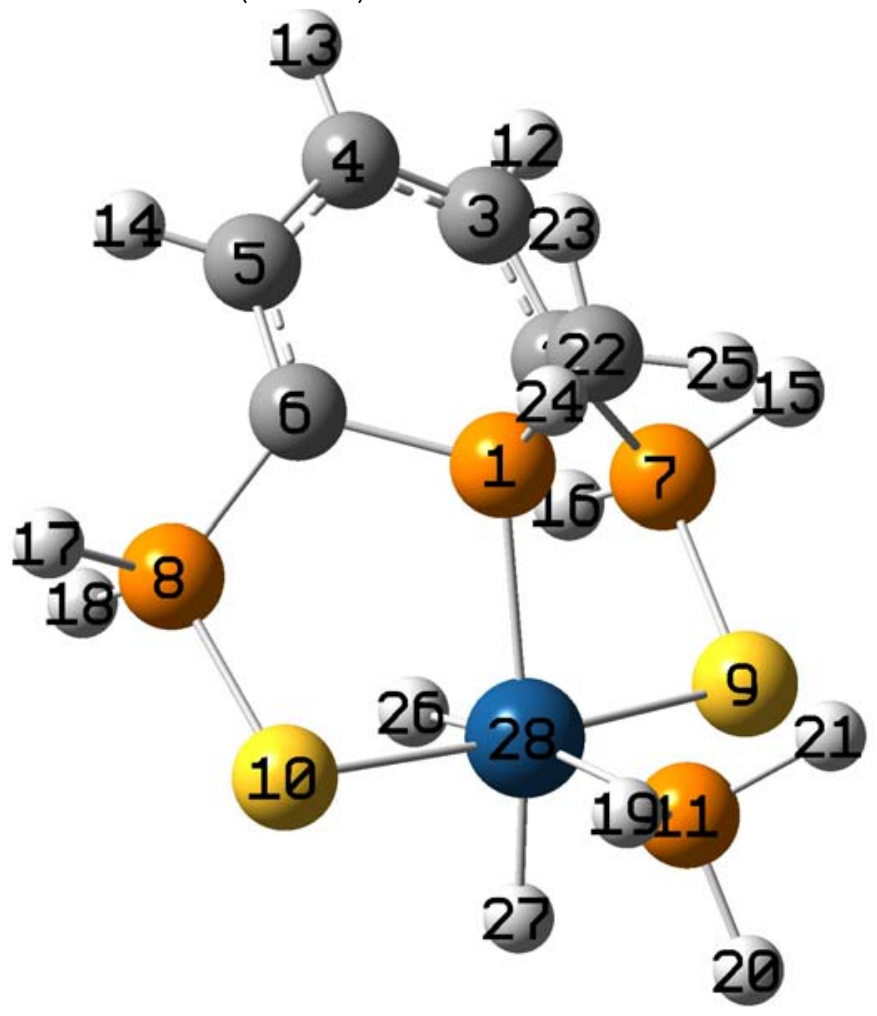

Optimized geometry:

\begin{tabular}{|c|c|c|c|c|c|}
\hline \multirow{2}{*}{$\begin{array}{l}\text { Center } \\
\text { Number }\end{array}$} & \multirow{2}{*}{$\begin{array}{l}\text { Atomic } \\
\text { Number }\end{array}$} & \multirow{2}{*}{$\begin{array}{c}\text { Atomic } \\
\text { Type }\end{array}$} & \multicolumn{3}{|c|}{ Coordinates (Angstroms) } \\
\hline & & & $\mathrm{X}$ & $\mathrm{Y}$ & $\mathrm{Z}$ \\
\hline 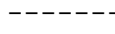 & & & & & - \\
\hline 1 & 15 & 0 & 0.896475 & 0.819742 & 0.297716 \\
\hline 2 & 6 & 0 & 0.889971 & 0.811088 & 2.095377 \\
\hline 3 & 6 & 0 & 2.108295 & 0.847225 & 2.770463 \\
\hline 4 & 6 & 0 & 3.357748 & 0.656253 & 2.150432 \\
\hline 5 & 6 & 0 & 3.471189 & 0.042357 & 0.888777 \\
\hline 6 & 6 & 0 & 2.415390 & -0.090413 & -0.011130 \\
\hline 7 & 15 & 0 & -0.646565 & 0.277231 & 2.782041 \\
\hline 8 & 15 & 0 & 2.211248 & -1.414010 & -1.161627 \\
\hline 9 & 16 & 0 & -2.186977 & 0.290889 & 1.447772 \\
\hline 10 & 16 & 0 & 0.485450 & -1.302575 & -2.240539 \\
\hline 11 & 15 & 0 & -1.953871 & 1.134376 & -1.910663 \\
\hline 12 & 1 & 0 & 2.116884 & 0.934202 & 3.859888 \\
\hline 13 & 1 & 0 & 4.253517 & 0.757616 & 2.755842 \\
\hline 14 & 1 & 0 & 4.430409 & -0.431601 & 0.665744 \\
\hline 15 & 1 & 0 & -0.996569 & 1.058974 & 3.906788 \\
\hline 16 & 1 & 0 & -0.510635 & -0.996168 & 3.391905 \\
\hline 17 & 1 & 0 & 3.310396 & -1.489424 & -2.047616 \\
\hline 18 & 1 & 0 & 2.326201 & -2.669024 & -0.510638 \\
\hline 19 & 1 & 0 & -1.318614 & 1.508014 & -3.121833 \\
\hline 20 & 1 & 0 & -3.224198 & 0.851456 & -2.464215 \\
\hline 21 & 1 & 0 & -2.259664 & 2.444011 & -1.459728 \\
\hline 22 & 6 & 0 & 1.316261 & 2.559335 & -0.140949 \\
\hline 23 & 1 & 0 & 2.221212 & 2.886405 & 0.381313 \\
\hline 24 & 1 & 0 & 1.478262 & 2.627109 & -1.222555 \\
\hline 25 & 1 & 0 & 0.481078 & 3.210689 & 0.139876 \\
\hline 26 & 1 & 0 & -0.397931 & -1.637916 & 0.417559 \\
\hline 27 & 1 & 0 & -2.071254 & -1.501805 & -0.850533 \\
\hline 28 & 77 & 0 & -0.929573 & -0.423126 & -0.490745 \\
\hline
\end{tabular}

Basis set A

Selected geometrical parameters : H-H 2.150, Ir-H1 1.632, Ir-H2 1.596, Ir-S 2.414, Ir-P1 2.308, Ir-P4 2.357, S1-Ir-S2 172.5, P1-Ir-P4106.0, P1-Ir-H1172.1, P4-Ir-H2 165.4. Frequencies:

\begin{tabular}{llr} 
& & \multicolumn{1}{c}{1} \\
& & \multicolumn{1}{c}{ A } \\
Frequencies -- & 30.8657 \\
Red. masses -- & 1.1437 \\
Frc consts -- & 0.0006 \\
IR Inten -- & 0.1215
\end{tabular}

2
A
43.8449
3.3576
0.0038
0.8827

3

A

68.0187

4.1575

0.0113

1.7185 
$\left[\operatorname{Ir}\left(\mathrm{SPS}\left(\mathrm{PH}_{3}\right)(\mathrm{H})_{2}\right]\right.$ anti-SS (IVb)



Basis set $A$

Selected geometrical parameters : H-H 1.786, Ir-H1 1.551, Ir-H2 1.567, Ir-S1 2.410, Ir-S2 4.735, Ir-P1 2.312, Ir-P4 2.302, S1-Ir-H1 137.5, S1-Ir-H2 152.6, P1-Ir-P4 1723.0 . Frequencies:

\begin{tabular}{|c|c|c|c|c|}
\hline \multirow[t]{2}{*}{$x^{2}$} & & 1 & 2 & 3 \\
\hline & & A & A & A \\
\hline Frequencies & -- & 26.8151 & 42.1531 & 55.8303 \\
\hline Red. masses & -- & 9.0553 & 8.5555 & 1.0947 \\
\hline Frc consts & -- & 0.0038 & 0.0090 & 0.0020 \\
\hline IR Inten & -- & 0.6502 & 4.1827 & 0.1564 \\
\hline
\end{tabular}


$\left[\operatorname{Ir}\left(\mathrm{SPS}\left(\mathrm{PH}_{3}\right)(\mathrm{H})_{2}\right]\right.$ trans $(\mathrm{Vb})$

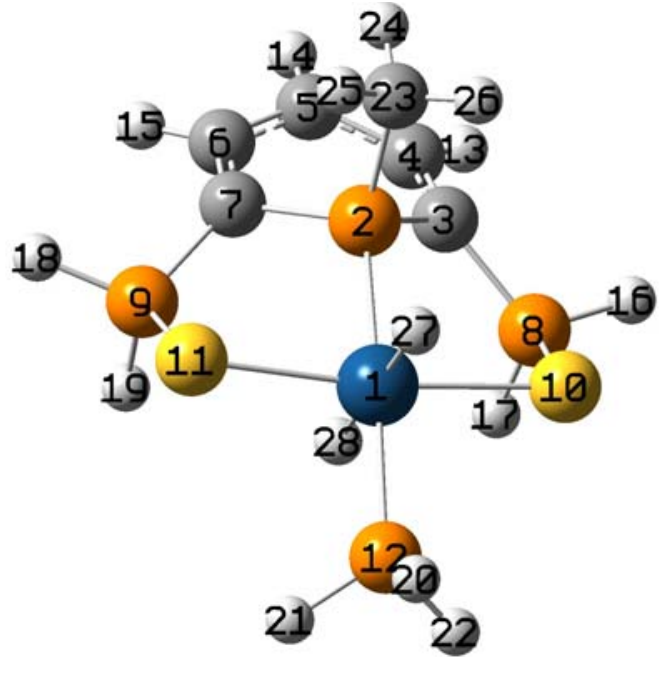

Optimized geometry:

\begin{tabular}{|c|c|c|c|c|c|}
\hline \multirow{2}{*}{$\begin{array}{l}\text { Center } \\
\text { Number }\end{array}$} & \multirow{2}{*}{$\begin{array}{l}\text { Atomic } \\
\text { Number }\end{array}$} & \multirow{2}{*}{$\begin{array}{l}\text { Atomic } \\
\text { Type }\end{array}$} & \multicolumn{3}{|c|}{ Coordinates (Angstroms) } \\
\hline & & & $\mathrm{X}$ & Y & Z \\
\hline & --- & & & & ---------- \\
\hline 1 & 77 & 0 & -0.212236 & -0.324920 & -0.940736 \\
\hline 2 & 15 & 0 & -0.196436 & -0.305955 & 1.340116 \\
\hline 3 & 6 & 0 & 1.488839 & -0.235258 & 1.950087 \\
\hline 4 & 6 & 0 & 1.738610 & 0.414521 & 3.156153 \\
\hline 5 & 6 & 0 & 0.798162 & 1.221220 & 3.824470 \\
\hline 6 & 6 & 0 & -0.323224 & 1.752178 & 3.159317 \\
\hline 7 & 6 & 0 & -0.817398 & 1.260961 & 1.953624 \\
\hline 8 & 15 & 0 & 2.644227 & -0.593570 & 0.667722 \\
\hline 9 & 15 & 0 & -1.618244 & 2.171807 & 0.674262 \\
\hline 10 & 16 & 0 & 1.783787 & -1.694032 & -0.809979 \\
\hline 11 & 16 & 0 & -2.275683 & 0.939652 & -0.803757 \\
\hline 12 & 15 & 0 & -0.108801 & -0.160001 & -3.232063 \\
\hline 13 & 1 & 0 & 2.745160 & 0.384431 & 3.580045 \\
\hline 14 & 1 & 0 & 1.069628 & 1.637369 & 4.789893 \\
\hline 15 & 1 & 0 & -0.760009 & 2.658480 & 3.585423 \\
\hline 16 & 1 & 0 & 3.765401 & -1.271195 & 1.200128 \\
\hline 17 & 1 & 0 & 3.238397 & 0.583361 & 0.150101 \\
\hline 18 & 1 & 0 & -2.692823 & 2.918727 & 1.210037 \\
\hline 19 & 1 & 0 & -0.786765 & 3.194781 & 0.156282 \\
\hline 20 & 1 & 0 & -0.722899 & -1.104454 & -4.088968 \\
\hline 21 & 1 & 0 & -0.629125 & 1.008645 & -3.834110 \\
\hline 22 & 1 & 0 & 1.169111 & -0.157995 & -3.836938 \\
\hline 23 & 6 & 0 & -1.043480 & -1.613838 & 2.298217 \\
\hline 24 & 1 & 0 & -0.932118 & -1.444735 & 3.374241 \\
\hline 25 & 1 & 0 & -2.103725 & -1.609984 & 2.025434 \\
\hline 26 & 1 & 0 & -0.608445 & -2.580077 & 2.023136 \\
\hline 27 & 1 & 0 & -1.109818 & -1.708261 & -1.004987 \\
\hline 28 & 1 & 0 & 0.710295 & 1.096889 & -0.875005 \\
\hline
\end{tabular}

Basis set A

Selected geometrical parameters : Ir-H1 1.650, Ir-H2 1.696, Ir-S1 2.424, Ir-S2 2.424, Ir-P1 2.281, Ir-P4 2.300, H1-Ir-H2 180.0, S1-Ir-S2 173.0, P1-Ir-P4 174.5.

Frequencies:

\begin{tabular}{|c|c|c|c|c|}
\hline & & 1 & 2 & 3 \\
\hline & & A & A & A \\
\hline Frequencies & -- & 65.3230 & 67.6699 & 77.1967 \\
\hline Red. masses & -- & 5.5802 & 3.6460 & 1.1352 \\
\hline Frc consts & -- & 0.0140 & 0.0098 & 0.0040 \\
\hline IR Inten & -- & 0.9708 & 0.6892 & 0.0628 \\
\hline
\end{tabular}


$\left[\operatorname{Ir}\left(\mathrm{SPS}\left(\mathrm{PH}_{3}\right)(\mathrm{H})_{2}\right] \quad M H \ldots S H \quad(\mathbf{V I b})\right.$

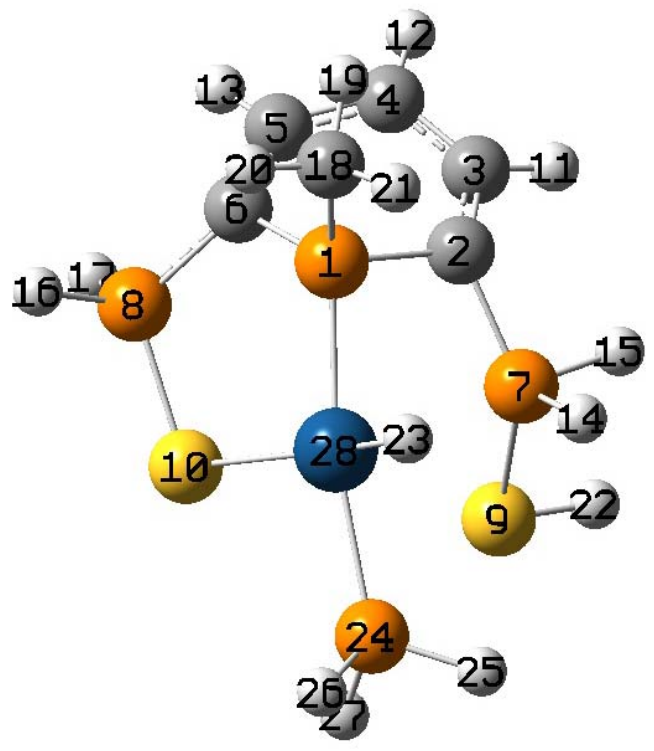

Optimized geometry:

\begin{tabular}{|c|c|c|c|c|c|}
\hline \multirow{2}{*}{$\begin{array}{l}\text { Center } \\
\text { Number }\end{array}$} & \multirow{2}{*}{$\begin{array}{l}\text { Atomic } \\
\text { Number }\end{array}$} & \multirow{2}{*}{$\begin{array}{l}\text { Atomic } \\
\text { Type }\end{array}$} & \multicolumn{3}{|c|}{ Coordinates (Angstroms) } \\
\hline & & & $\mathrm{x}$ & Y & Z \\
\hline 1 & 15 & 0 & -1.338468 & -0.023771 & 0.963546 \\
\hline 2 & 6 & 0 & -1.358924 & 1.667162 & 0.394785 \\
\hline 3 & 6 & 0 & -2.473249 & 2.180728 & -0.250260 \\
\hline 4 & 6 & 0 & -3.597357 & 1.400177 & -0.601560 \\
\hline 5 & 6 & 0 & -3.505781 & 0.007769 & -0.731891 \\
\hline 6 & 6 & 0 & -2.468418 & -0.784465 & -0.208049 \\
\hline 7 & 15 & 0 & 0.340777 & 2.118397 & 0.044223 \\
\hline 8 & 15 & 0 & -1.720772 & -2.107644 & -1.079910 \\
\hline 9 & 16 & 0 & 1.466321 & 1.867942 & -1.790221 \\
\hline 10 & 16 & 0 & 0.285710 & -1.840104 & -1.400956 \\
\hline 11 & 1 & 0 & -2.439523 & 3.207392 & -0.622169 \\
\hline 12 & 1 & 0 & -4.457960 & 1.897236 & -1.039580 \\
\hline 13 & 1 & 0 & -4.253819 & -0.465838 & -1.370794 \\
\hline 14 & 1 & 0 & 1.127063 & 2.555103 & 1.131005 \\
\hline 15 & 1 & 0 & 0.009068 & 3.471148 & -0.370114 \\
\hline 16 & 1 & 0 & -1.921507 & -3.361211 & -0.454979 \\
\hline 17 & 1 & 0 & -2.441715 & -2.303383 & -2.279572 \\
\hline 18 & 6 & 0 & -2.122860 & -0.116753 & 2.616849 \\
\hline 19 & 1 & 0 & -3.144905 & 0.273567 & 2.574435 \\
\hline 20 & 1 & 0 & -2.144314 & -1.157663 & 2.956198 \\
\hline 21 & 1 & 0 & -1.530231 & 0.476410 & 3.320582 \\
\hline 22 & 1 & 0 & 1.368684 & 3.148479 & -2.226547 \\
\hline 23 & 1 & 0 & 1.217004 & 0.253465 & 1.920808 \\
\hline 24 & 15 & 0 & 3.122964 & -0.338781 & 0.149306 \\
\hline 25 & 1 & 0 & 3.845101 & 0.840819 & 0.437372 \\
\hline 26 & 1 & 0 & 3.898259 & -1.232036 & 0.930574 \\
\hline 27 & 1 & 0 & 3.719656 & -0.624196 & -1.102558 \\
\hline 28 & 77 & 0 & 0.859852 & -0.367213 & 0.503603 \\
\hline
\end{tabular}

Basis set $A$

Selected geometrical parameters : Ir-H1 1.588, S2-H2 1.356, Ir-S1 2.475, Ir-S2 3.260, Ir-P1 2.272, Ir-P4 2.291, S1-Ir-H1 166.3, P1-Ir-P4 170.2.

Frequencies:

Frequencies --

Red. masses --

Frc consts --

IR Inten
A

43.3389

8.5698

0.0095

0.7009
2

A 66.5522

10.2828

0.0268

1.5257
3

7.8643

5.4061

0.0193

0.9004 
$\left[\operatorname{Ir}\left(\mathrm{SPS}\left(\mathrm{PH}_{3}\right)(\mathrm{H})_{2}\right] \quad(\mathbf{T S}-\mathbf{I b})\right.$

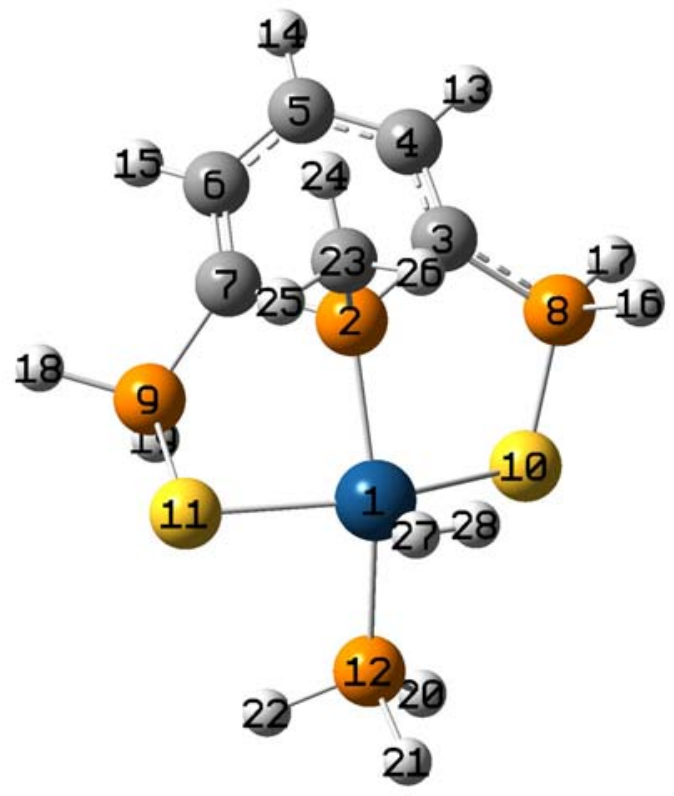

Optimized geometry:

\begin{tabular}{|c|c|c|c|c|c|}
\hline \multirow{2}{*}{$\begin{array}{l}\text { Center } \\
\text { Number }\end{array}$} & \multirow{2}{*}{$\begin{array}{l}\text { Atomic } \\
\text { Number }\end{array}$} & \multirow{2}{*}{$\begin{array}{c}\text { Atomic } \\
\text { Type }\end{array}$} & \multicolumn{3}{|c|}{ Coordinates (Angstroms) } \\
\hline & & & $\mathrm{X}$ & $\mathrm{Y}$ & Z \\
\hline-- & -- & & & & --- \\
\hline 1 & 77 & 0 & -0.211239 & -0.400166 & -0.970977 \\
\hline 2 & 15 & 0 & -0.189565 & -0.422547 & 1.315943 \\
\hline 3 & 6 & 0 & 1.446709 & -0.392755 & 2.058169 \\
\hline 4 & 6 & 0 & 1.645720 & 0.301393 & 3.256980 \\
\hline 5 & 6 & 0 & 0.722158 & 1.188508 & 3.824183 \\
\hline 6 & 6 & 0 & -0.365887 & 1.702874 & 3.086994 \\
\hline 7 & 6 & 0 & -0.822858 & 1.153054 & 1.900555 \\
\hline 8 & 15 & 0 & 2.672468 & -0.915193 & 0.928641 \\
\hline 9 & 15 & 0 & -1.686100 & 1.962532 & 0.589724 \\
\hline 10 & 16 & 0 & 2.235297 & -0.335984 & -0.972046 \\
\hline 11 & 16 & 0 & -2.410593 & 0.611929 & -0.739339 \\
\hline 12 & 15 & 0 & -0.150794 & 0.311921 & -3.148086 \\
\hline 13 & 1 & 0 & 2.611446 & 0.216023 & 3.758483 \\
\hline 14 & 1 & 0 & 0.974464 & 1.659681 & 4.769090 \\
\hline 15 & 1 & 0 & -0.796208 & 2.643583 & 3.437656 \\
\hline 16 & 1 & 0 & 2.878200 & -2.318765 & 0.977305 \\
\hline 17 & 1 & 0 & 3.941385 & -0.456488 & 1.351212 \\
\hline 18 & 1 & 0 & -2.725236 & 2.734332 & 1.178738 \\
\hline 19 & 1 & 0 & -0.922218 & 2.968634 & -0.043758 \\
\hline 20 & 1 & 0 & 1.047540 & 0.878807 & -3.647158 \\
\hline 21 & 1 & 0 & -0.403910 & -0.538156 & -4.258389 \\
\hline 22 & 1 & 0 & -1.040208 & 1.349413 & -3.514518 \\
\hline 23 & 6 & 0 & -1.137584 & -1.700840 & 2.237257 \\
\hline 24 & 1 & 0 & -1.086277 & -1.515632 & 3.315421 \\
\hline 25 & 1 & 0 & -2.181258 & -1.664765 & 1.906826 \\
\hline 26 & 1 & 0 & -0.726784 & -2.692093 & 2.015161 \\
\hline 27 & 1 & 0 & -0.960267 & -2.114712 & -1.282579 \\
\hline 28 & 1 & 0 & -0.160193 & -2.316306 & -1.294225 \\
\hline
\end{tabular}

Basis set $A$

Selected geometrical parameters : H-H 0.825, Ir-H1 1.897, Ir-H2, 1.944, S1-Ir 2.432, S2-Ir 2.447, P1-Ir 2.287, P4-Ir 2.291, S-Ir-S 153.3, P1-Ir-P4 162.3.

$\mathrm{SCF}$ Done: $\mathrm{E}(\mathrm{RB}+\mathrm{HF}-\mathrm{PW} 91)=-2503.82975380$

A.U. after 1 cycles

Sum of electronic and zero-point Energies=

Sum of electronic and thermal Energies=

$-2503.642599$

$-2503.624321$

$-2503.623377$

Sum of electronic and thermal Enthalpies=
Sum of electronic and thermal Free Energies

$-2503.688473$

Total
E (Thermal) $\mathrm{KCal} / \mathrm{Mol}$

128.911
$C V$

Cal/Mol-Kelvin 69.273
$\mathrm{S}$

$\mathrm{Cal/Mol-Kelvin}$ 
Frequencies:

A

Frequencies -- $\quad-105.6381$

Red. masses -- 7.3078

Frc consts -- 0.0480

IR Inten -- 0.2051
2

A

54.8914

5.6288

0.0100

1.6189
3

2.8130

5.4837

0.0127

4.5924 
$[\mathrm{Pd}(\mathrm{SPS})(\mathrm{Cl})]$

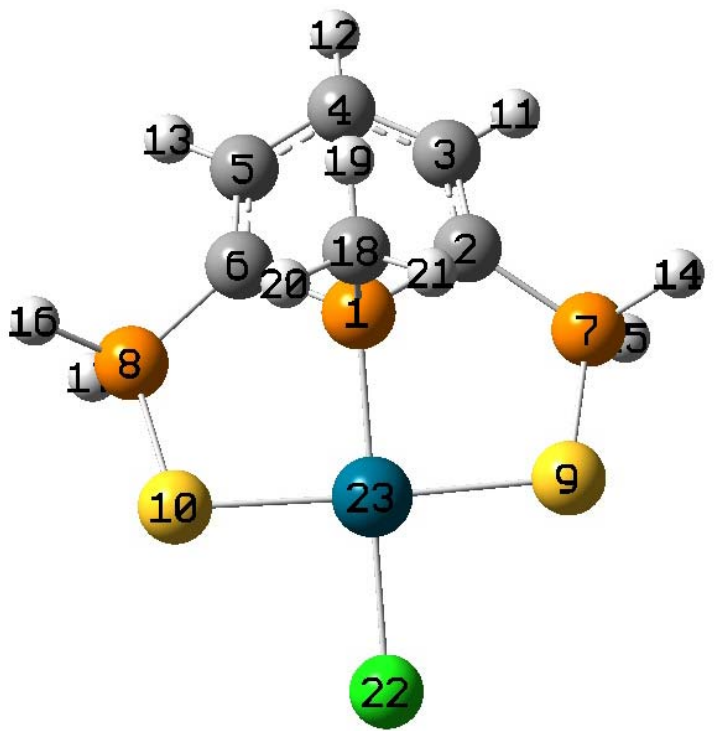

Optimized geometry:

\begin{tabular}{|c|c|c|c|c|c|}
\hline \multirow{2}{*}{$\begin{array}{l}\text { Center } \\
\text { Number }\end{array}$} & \multirow{2}{*}{$\begin{array}{l}\text { Atomic } \\
\text { Number }\end{array}$} & \multirow{2}{*}{$\begin{array}{l}\text { Atomic } \\
\text { Type }\end{array}$} & \multicolumn{3}{|c|}{ Coordinates (Angstroms) } \\
\hline & & & $\mathrm{x}$ & $\mathrm{Y}$ & Z \\
\hline 1 & 15 & 0 & .757632 & .836552 & .234590 \\
\hline 2 & 6 & 0 & .736339 & .853456 & 2.024680 \\
\hline 3 & 6 & 0 & 1.928180 & .774948 & 2.741346 \\
\hline 4 & 6 & 0 & 3.185214 & .506300 & 2.179360 \\
\hline 5 & 6 & 0 & 3.328800 & .032109 & .866860 \\
\hline 6 & 6 & 0 & 2.315547 & .015898 & -.088815 \\
\hline 7 & 15 & 0 & -.891503 & .703250 & 2.687298 \\
\hline 8 & 15 & 0 & 2.272768 & -.974974 & -1.547525 \\
\hline 9 & 16 & 0 & -2.295489 & .443120 & 1.238612 \\
\hline 10 & 16 & 0 & .430825 & -1.002831 & -2.410084 \\
\hline 11 & 1 & 0 & 1.888918 & .852682 & 3.829429 \\
\hline 12 & 1 & 0 & 4.049667 & .483246 & 2.834418 \\
\hline 13 & 1 & 0 & 4.289862 & -.420693 & .616183 \\
\hline 14 & 1 & 0 & -1.239241 & 1.823983 & 3.476690 \\
\hline 15 & 1 & 0 & -.900254 & -.305208 & 3.682685 \\
\hline 16 & 1 & 0 & 3.226035 & -.544251 & -2.499295 \\
\hline 17 & 1 & 0 & 2.788720 & -2.261717 & -1.254359 \\
\hline 18 & 6 & 0 & .888677 & 2.569145 & -.354102 \\
\hline 19 & 1 & 0 & 1.757038 & 3.058462 & .100827 \\
\hline 20 & 1 & 0 & .990714 & 2.572011 & -1.444674 \\
\hline 21 & 1 & 0 & -.024815 & 3.110613 & -.085566 \\
\hline 22 & 17 & 0 & -2.837183 & -1.393742 & -1.567636 \\
\hline 23 & 46 & 0 & -.981302 & -.276167 & -.623791 \\
\hline
\end{tabular}

Basis set A

Frequencies:

\begin{tabular}{|c|c|c|c|c|}
\hline & & 1 & 2 & 3 \\
\hline & & A & A & A \\
\hline Frequencies & -- & 19.1516 & 33.6992 & 52.7706 \\
\hline Red. masses & -- & 6.7463 & 4.1663 & 13.8556 \\
\hline Frc consts & -- & .0015 & .0028 & .0227 \\
\hline IR Inten & - - & .0197 & 5.8361 & 1.0799 \\
\hline
\end{tabular}


$\left[\mathrm{Pd}(\mathrm{SPS})(\mathrm{Cl})(\mathrm{H})_{2}\right]$ syn-SS (Ic)

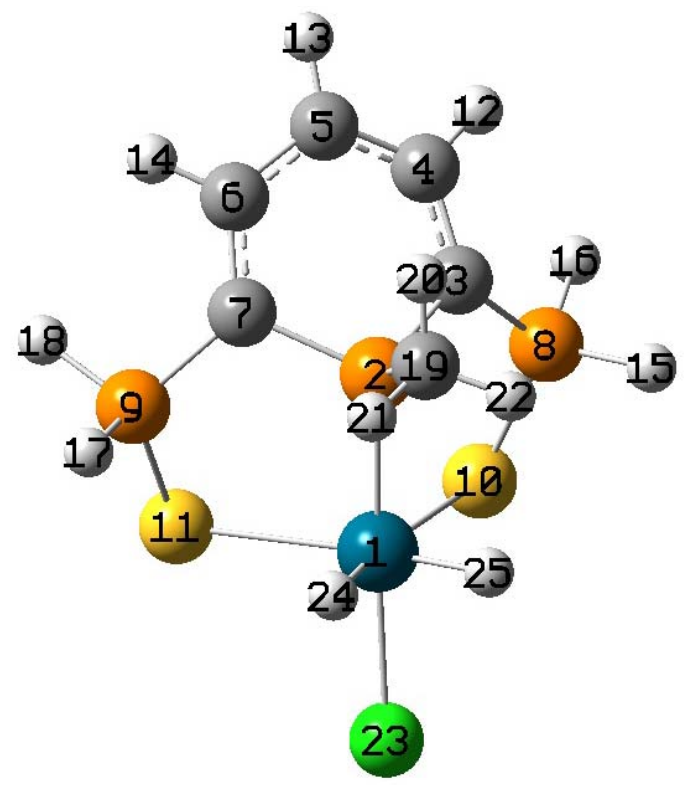

Optimized geometry:

\begin{tabular}{|c|c|c|c|c|c|}
\hline \multirow{2}{*}{$\begin{array}{l}\text { Center } \\
\text { Number }\end{array}$} & \multirow{2}{*}{$\begin{array}{l}\text { Atomic } \\
\text { Number }\end{array}$} & \multirow{2}{*}{$\begin{array}{l}\text { Atomic } \\
\text { Type }\end{array}$} & \multicolumn{3}{|c|}{ Coordinates (Angstroms) } \\
\hline & & & $\mathrm{X}$ & $\mathrm{Y}$ & Z \\
\hline & & & & (1) & 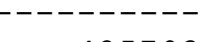 \\
\hline 1 & 46 & 0 & -1.231296 & -.000038 & .425793 \\
\hline 2 & 15 & 0 & 1.032101 & .000016 & .864165 \\
\hline 3 & 6 & 0 & 1.845863 & -1.386623 & .088765 \\
\hline 4 & 6 & 0 & 3.160376 & -1.223116 & -.350478 \\
\hline 5 & 6 & 0 & 3.844293 & .000023 & -.410454 \\
\hline 6 & 6 & 0 & 3.160358 & 1.223145 & -.350501 \\
\hline 7 & 6 & 0 & 1.845817 & 1.386630 & .088696 \\
\hline 8 & 15 & 0 & .756717 & -2.652055 & -.496891 \\
\hline 9 & 15 & 0 & .756722 & 2.652071 & -.497007 \\
\hline 10 & 16 & 0 & -.961517 & -1.947315 & -1.257579 \\
\hline 11 & 16 & 0 & -.961708 & 1.947367 & -1.257330 \\
\hline 12 & 1 & 0 & 3.675172 & -2.090686 & -.766449 \\
\hline 13 & 1 & 0 & 4.873425 & .000030 & -.753976 \\
\hline 14 & 1 & 0 & 3.675149 & 2.090719 & -.766467 \\
\hline 15 & 1 & 0 & .527061 & -3.611484 & .518697 \\
\hline 16 & 1 & 0 & 1.515290 & -3.416532 & -1.410872 \\
\hline 17 & 1 & 0 & .527303 & 3.611736 & .518417 \\
\hline 18 & 1 & 0 & 1.515218 & 3.416315 & -1.411248 \\
\hline 19 & 6 & 0 & 1.475510 & .000067 & 2.641472 \\
\hline 20 & 1 & 0 & 2.565287 & .000071 & 2.748100 \\
\hline 21 & 1 & 0 & 1.059382 & .891376 & 3.122174 \\
\hline 22 & 1 & 0 & 1.059377 & -.891199 & 3.122249 \\
\hline 23 & 17 & 0 & -3.583446 & -.000024 & .253939 \\
\hline 24 & 1 & 0 & -1.419683 & .998251 & 1.557788 \\
\hline 25 & 1 & 0 & -1.419579 & -.998507 & 1.557658 \\
\hline
\end{tabular}

Basis set $A$

Selected geometrical parameters : H-H 1.997, Pd-H1 1.521, Pd-S1 1.588, Pd-P1 2.305, Pd-Cl 2.358, S-Pd-S 96.6, P1-Pd-Cl 173.2.

Frequencies:

\begin{tabular}{llr} 
& \multicolumn{1}{c}{1} \\
& \multicolumn{1}{c}{ A } \\
Frequencies -- & 47.9073 \\
Red. masses -- & 7.5830 \\
Frc consts -- & .0103 \\
IR Inten -- & .0622
\end{tabular}
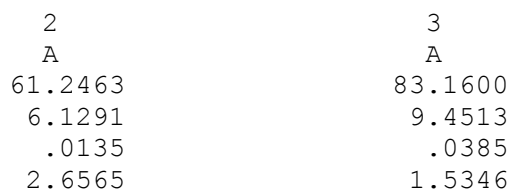
$\left[\mathrm{Pd}(\mathrm{SPS})(\mathrm{Cl})(\mathrm{H})_{2}\right] \quad M H \ldots S H \quad(\mathrm{VIC})$

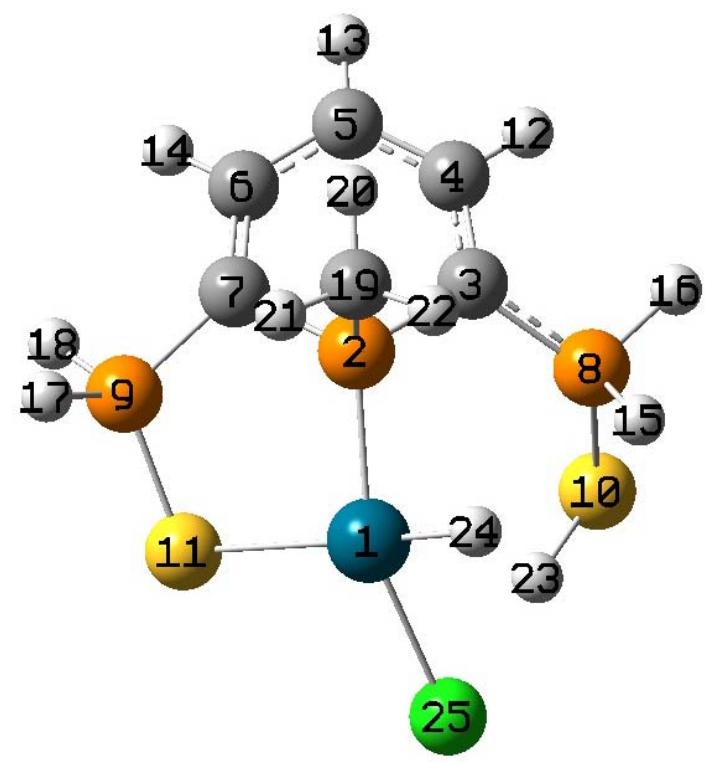

Optimized geometry:

\begin{tabular}{|c|c|c|c|c|c|}
\hline \multirow{2}{*}{$\begin{array}{l}\text { Center } \\
\text { Number }\end{array}$} & \multirow{2}{*}{$\begin{array}{l}\text { Atomic } \\
\text { Number }\end{array}$} & \multirow{2}{*}{$\begin{array}{l}\text { Atomic } \\
\text { Type }\end{array}$} & \multicolumn{3}{|c|}{ Coordinates (Angstroms) } \\
\hline & & & $\mathrm{X}$ & Y & Z \\
\hline 1 & 46 & 0 & 0.000000 & 0.000000 & 0.000000 \\
\hline 2 & 15 & 0 & -0.948278 & -1.997536 & 0.268213 \\
\hline 3 & 6 & 0 & -0.666651 & -2.944841 & -1.233560 \\
\hline 4 & 6 & 0 & -0.422403 & -4.331037 & -1.143989 \\
\hline 5 & 6 & 0 & -0.180755 & -5.016388 & 0.040371 \\
\hline 6 & 6 & 0 & 0.161984 & -4.339215 & 1.236610 \\
\hline 7 & 6 & 0 & -0.023156 & -2.988727 & 1.459649 \\
\hline 8 & 15 & 0 & -0.304889 & -2.072510 & -2.701045 \\
\hline 9 & 15 & 0 & 0.935054 & -2.022069 & 2.601502 \\
\hline 10 & 16 & 0 & 1.519816 & -1.170884 & -3.154376 \\
\hline 11 & 16 & 0 & 1.766230 & -0.391014 & 1.780645 \\
\hline 12 & 1 & 0 & -0.356755 & -4.906006 & -2.070046 \\
\hline 13 & 1 & 0 & -0.035547 & -6.091047 & -0.000926 \\
\hline 14 & 1 & 0 & 0.694927 & -4.926805 & 1.986373 \\
\hline 15 & 1 & 0 & -1.297695 & -1.125815 & -2.990560 \\
\hline 16 & 1 & 0 & -0.405206 & -3.018877 & -3.738523 \\
\hline 17 & 1 & 0 & 0.142771 & -1.708386 & 3.732056 \\
\hline 18 & 1 & 0 & 1.862106 & -2.919448 & 3.178348 \\
\hline 19 & 6 & 0 & -2.722378 & -2.225335 & 0.685120 \\
\hline 20 & 1 & 0 & -2.973915 & -3.290128 & 0.727118 \\
\hline 21 & 1 & 0 & -2.929878 & -1.760016 & 1.654643 \\
\hline 22 & 1 & 0 & -3.334061 & -1.731789 & -0.077087 \\
\hline 23 & 1 & 0 & 1.352848 & -0.041589 & -2.338888 \\
\hline 24 & 1 & 0 & -1.138259 & 0.231124 & -1.002842 \\
\hline 25 & 17 & 0 & 1.196590 & 1.730040 & -1.142755 \\
\hline
\end{tabular}

Basis set A

Selected geometrical parameters : Pd-H1 1.535, Pd-S1 2.538, Pd-P1 2.227, Pd-Cl 2.394, S2-H2 1.403, H2-Cl 2.143, S1-Pd-H1 176.2, P1-Pd-Cl 156.7.

Frequencies:

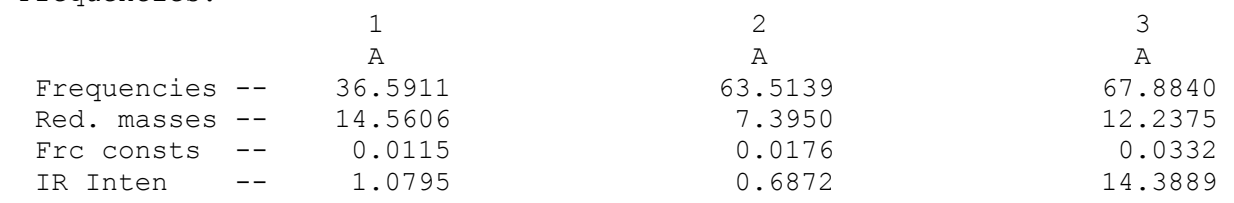


$\left[\mathrm{Pd}(\mathrm{SPS})(\mathrm{Cl})(\mathrm{H})_{2}\right]$ molecular (VIIC)

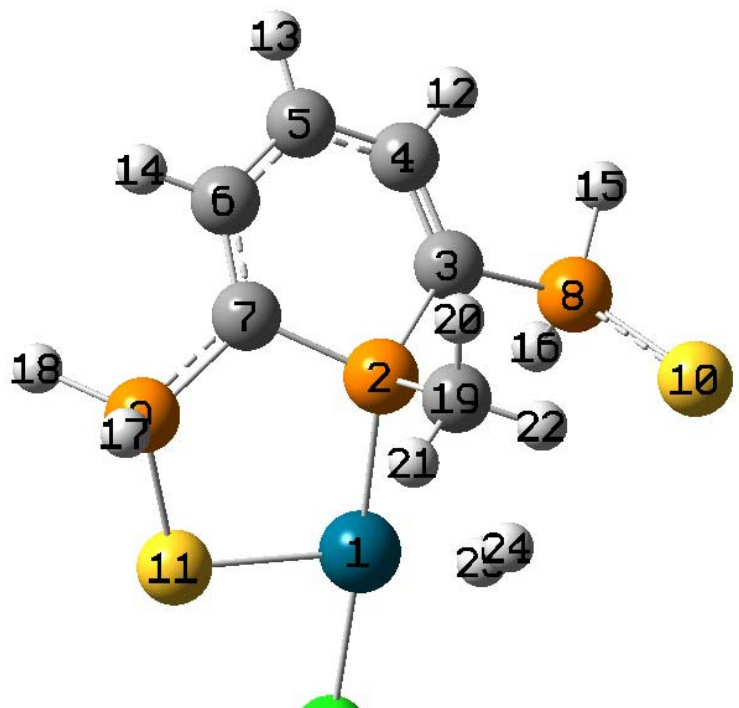

Optimized geometry:

\begin{tabular}{|c|c|c|c|c|c|}
\hline \multirow{2}{*}{$\begin{array}{l}\text { Center } \\
\text { Number }\end{array}$} & \multirow{2}{*}{$\begin{array}{l}\text { Atomic } \\
\text { Number }\end{array}$} & \multirow{2}{*}{$\begin{array}{l}\text { Atomic } \\
\text { Type }\end{array}$} & \multicolumn{3}{|c|}{ Coordinates (Angstroms) } \\
\hline & & & $\mathrm{X}$ & Y & Z \\
\hline 1 & 46 & 0 & -.413514 & -.054342 & -1.364727 \\
\hline 2 & 15 & 0 & -.079176 & -.195752 & .920540 \\
\hline 3 & 6 & 0 & 1.580409 & -.254663 & 1.587448 \\
\hline 4 & 6 & 0 & 1.928802 & .506776 & 2.691302 \\
\hline 5 & 6 & 0 & 1.129426 & 1.471079 & 3.335016 \\
\hline 6 & 6 & 0 & -.099987 & 1.859692 & 2.823676 \\
\hline 7 & 6 & 0 & -.729507 & 1.261574 & 1.722396 \\
\hline 8 & 15 & 0 & 2.807965 & -1.296063 & .792524 \\
\hline 9 & 15 & 0 & -2.111342 & 1.952918 & .906279 \\
\hline 10 & 16 & 0 & 2.338570 & -3.154329 & .368464 \\
\hline 11 & 16 & 0 & -2.095597 & 1.559728 & -1.103933 \\
\hline 12 & 1 & 0 & 2.940037 & .377524 & 3.081722 \\
\hline 13 & 1 & 0 & 1.538057 & 2.000287 & 4.188928 \\
\hline 14 & 1 & 0 & -.584865 & 2.720656 & 3.284854 \\
\hline 15 & 1 & 0 & 3.919122 & -1.096313 & 1.647892 \\
\hline 16 & 1 & 0 & 3.225210 & -.561457 & -.347012 \\
\hline 17 & 1 & 0 & -3.352075 & 1.529904 & 1.438343 \\
\hline 18 & 1 & 0 & -2.161706 & 3.330911 & 1.219292 \\
\hline 19 & 6 & 0 & -.985574 & -1.687006 & 1.502866 \\
\hline 20 & 1 & 0 & -.957386 & -1.724602 & 2.597039 \\
\hline 21 & 1 & 0 & -2.023575 & -1.645073 & 1.155246 \\
\hline 22 & 1 & 0 & -.501570 & -2.581369 & 1.097539 \\
\hline 23 & 17 & 0 & -.863164 & .135031 & -3.683683 \\
\hline 24 & 1 & 0 & .618744 & -1.515088 & -1.472123 \\
\hline 25 & 1 & 0 & .944751 & -.978827 & -1.990558 \\
\hline
\end{tabular}

Basis set $A$

Selected geometrical parameters : H1-H2 0.814, Pd-H1 1.792, Pd-H2 1.758, Pd-S1 2.346, Pd-S2 4.493, Pd-P1 2.314, Pd-Cl 2.370, P1-Pd-Cl 177.1, S1-Pd-H1 168.7, S1-Pd-H2 162.8 .

Frequencies:

\begin{tabular}{|c|c|c|c|c|}
\hline & & 1 & 2 & 3 \\
\hline & & A & A & A \\
\hline Frequencies & -- & 28.5256 & 36.0373 & 48.5180 \\
\hline Red. masses & -- & 9.8846 & 7.7131 & 7.8677 \\
\hline Frc consts & -- & .0047 & .0059 & .0109 \\
\hline IR Inten & -- & 1.3532 & 3.5599 & 2.7146 \\
\hline
\end{tabular}


$\left[\mathrm{Pd}(\mathrm{SPS})\left(\mathrm{PH}_{3}\right)\right]^{+}$

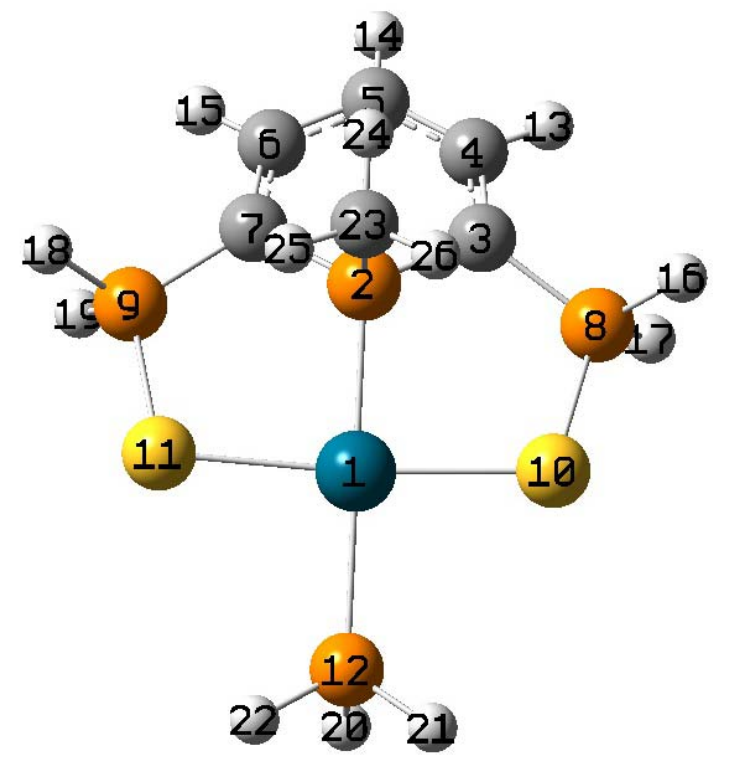

Optimized geometry:

\begin{tabular}{|c|c|c|c|c|c|}
\hline \multirow{2}{*}{$\begin{array}{l}\text { Center } \\
\text { Number }\end{array}$} & \multirow{2}{*}{$\begin{array}{l}\text { Atomic } \\
\text { Number }\end{array}$} & \multirow{2}{*}{$\begin{array}{c}\text { Atomic } \\
\text { Type }\end{array}$} & \multicolumn{3}{|c|}{ Coordinates (Angstroms) } \\
\hline & & & $\mathrm{X}$ & Y & Z \\
\hline 1 & 46 & 0 & -0.160789 & -0.232432 & -1.142765 \\
\hline 2 & 15 & 0 & -0.224189 & -0.315905 & 1.125971 \\
\hline 3 & 6 & 0 & 1.409317 & -0.396780 & 1.835743 \\
\hline 4 & 6 & 0 & 1.746988 & 0.365811 & 2.955861 \\
\hline 5 & 6 & 0 & 0.925847 & 1.329278 & 3.550897 \\
\hline 6 & 6 & 0 & -0.257522 & 1.773265 & 2.951628 \\
\hline 7 & 6 & 0 & -0.856963 & 1.194475 & 1.830958 \\
\hline 8 & 15 & 0 & 2.548288 & -1.291035 & 0.836251 \\
\hline 9 & 15 & 0 & -2.082015 & 1.960107 & 0.826474 \\
\hline 10 & 16 & 0 & 1.777692 & -1.631083 & -1.030875 \\
\hline 11 & 16 & 0 & -2.134893 & 1.116131 & -1.039142 \\
\hline 12 & 15 & 0 & -0.085150 & -0.131930 & -3.541188 \\
\hline 13 & 1 & 0 & 2.749037 & 0.254760 & 3.371049 \\
\hline 14 & 1 & 0 & 1.295405 & 1.858226 & 4.422055 \\
\hline 15 & 1 & 0 & -0.703420 & 2.678881 & 3.363760 \\
\hline 16 & 1 & 0 & 2.909747 & -2.544734 & 1.371164 \\
\hline 17 & 1 & 0 & 3.791878 & -0.620912 & 0.834101 \\
\hline 18 & 1 & 0 & -3.385469 & 1.875411 & 1.357868 \\
\hline 19 & 1 & 0 & -1.874003 & 3.357353 & 0.822145 \\
\hline 20 & 1 & 0 & 0.661406 & 0.929625 & -4.088743 \\
\hline 21 & 1 & 0 & 0.477924 & -1.208555 & -4.254709 \\
\hline 22 & 1 & 0 & -1.286979 & 0.030721 & -4.258432 \\
\hline 23 & 6 & 0 & -1.230617 & -1.747654 & 1.663496 \\
\hline 24 & 1 & 0 & -1.259084 & -1.784892 & 2.758150 \\
\hline 25 & 1 & 0 & -2.248466 & -1.650806 & 1.270918 \\
\hline 26 & 1 & 0 & -0.792768 & -2.672909 & 1.274004 \\
\hline
\end{tabular}

Basis set A

Frequencies:

\begin{tabular}{llr} 
& & 1 \\
& & \multicolumn{1}{c}{ A } \\
Frequencies -- & 26.9337 \\
Red. masses -- & 5.0570 \\
Frc consts -- & 0.0022 \\
IR Inten & -- & 0.4704
\end{tabular}

\begin{tabular}{l}
\multicolumn{1}{l}{} \\
A \\
38.4454 \\
4.4735 \\
0.0039 \\
2.9974
\end{tabular}

3

A

49.4653

5.6990

0.0082

8.6583 
$\left[\mathrm{Pd}(\mathrm{SPS})\left(\mathrm{PH}_{3}\right)(\mathrm{H})_{2}\right]^{+}$syn-SS $(\mathbf{I d})$

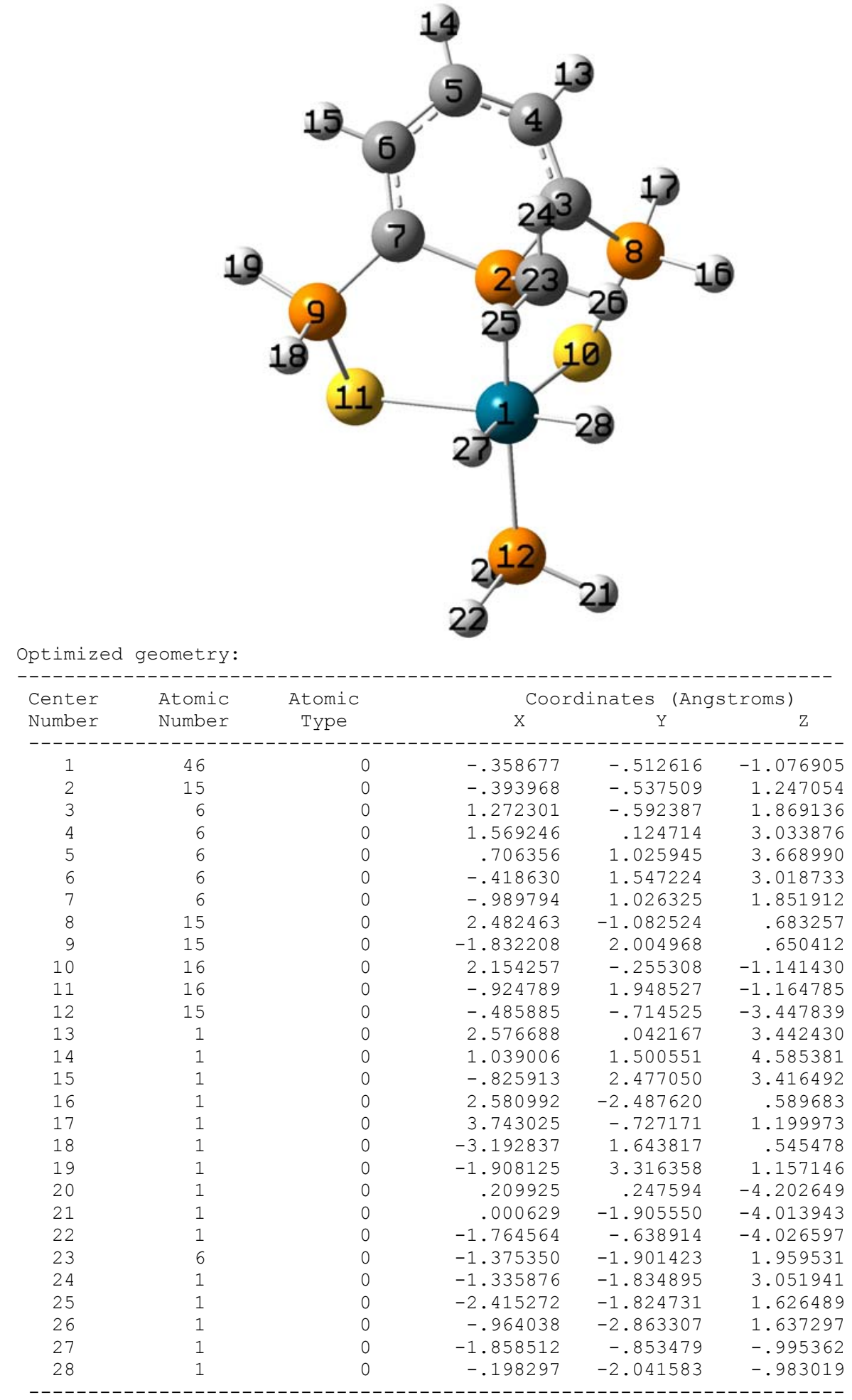

Basis set A

Selected geometrical parameters : $\mathrm{H}-\mathrm{H} 2.042, \mathrm{Pd}-\mathrm{H} 11.540 \mathrm{Pd}-\mathrm{S} 2.527, \mathrm{Pd}-\mathrm{P} 12.324, \mathrm{Pd}-\mathrm{P} 42.383$, S-Pd-S 97.0, P1-Pd-P4 172.3.

Frequencies:

\begin{tabular}{|c|c|c|c|c|}
\hline & & 1 & 2 & 3 \\
\hline & & A & A & A \\
\hline Frequencies & -- & 47.6928 & 59.3309 & 66.6427 \\
\hline Red. masses & -- & 3.0214 & 5.0973 & 1.2882 \\
\hline Frc consts & -- & .0040 & .0106 & .0034 \\
\hline IR Inten & -- & 1.0443 & .0557 & .1782 \\
\hline
\end{tabular}


$\left[\mathrm{Pd}(\mathrm{SPS})\left(\mathrm{PH}_{3}\right)(\mathrm{H})_{2}\right]^{+} \quad M H \ldots . . . S H \quad(\mathbf{V I d})$

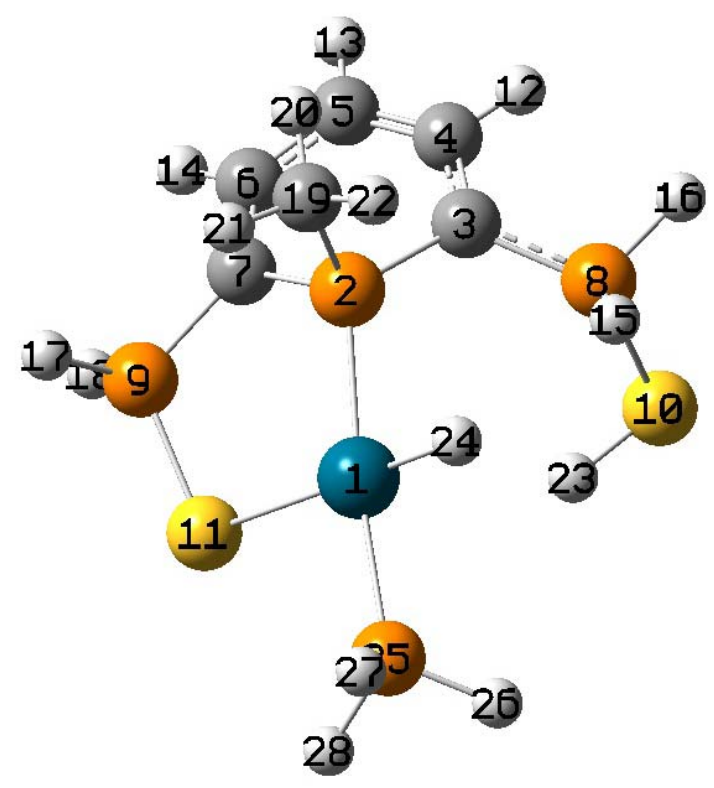

Optimized geometry:

\begin{tabular}{|c|c|c|c|c|c|}
\hline \multirow{2}{*}{$\begin{array}{l}\text { Center } \\
\text { Number }\end{array}$} & \multirow{2}{*}{$\begin{array}{l}\text { Atomic } \\
\text { Number }\end{array}$} & \multirow{2}{*}{$\begin{array}{l}\text { Atomic } \\
\text { Type }\end{array}$} & \multicolumn{3}{|c|}{ Coordinates (Angstroms) } \\
\hline & & & $\mathrm{x}$ & $\mathrm{Y}$ & $\mathrm{z}$ \\
\hline 1 & 46 & 0 & 0.062333 & 0.042791 & 0.021308 \\
\hline 2 & 15 & 0 & 0.006659 & 0.012171 & 2.322502 \\
\hline 3 & 6 & 0 & 1.573536 & 0.008895 & 3.200788 \\
\hline 4 & 6 & 0 & 1.705752 & 0.734279 & 4.410083 \\
\hline 5 & 6 & 0 & 0.770373 & 1.621720 & 4.911492 \\
\hline 6 & 6 & 0 & -0.310889 & 2.094197 & 4.130780 \\
\hline 7 & 6 & 0 & -0.711463 & 1.552532 & 2.924769 \\
\hline 8 & 15 & 0 & 2.977428 & -0.607114 & 2.401450 \\
\hline 9 & 15 & 0 & -1.769698 & 2.382396 & 1.772192 \\
\hline 10 & 16 & 0 & 3.916251 & 0.500741 & 0.878330 \\
\hline 11 & 16 & 0 & -1.174335 & 2.160393 & -0.149560 \\
\hline 12 & 1 & 0 & 2.631841 & 0.620836 & 4.975259 \\
\hline 13 & 1 & 0 & 0.964569 & 2.100897 & 5.864730 \\
\hline 14 & 1 & 0 & -0.804149 & 2.998466 & 4.488865 \\
\hline 15 & 1 & 0 & 2.758407 & -1.886036 & 1.867262 \\
\hline 16 & 1 & 0 & 4.010288 & -0.765866 & 3.337822 \\
\hline 17 & 1 & 0 & -3.107966 & 1.961310 & 1.932221 \\
\hline 18 & 1 & 0 & -1.850209 & 3.732844 & 2.167485 \\
\hline 19 & 6 & 0 & -0.991705 & -1.354382 & 3.031547 \\
\hline 20 & 1 & 0 & -1.043238 & -1.257142 & 4.120777 \\
\hline 21 & 1 & 0 & -2.004232 & -1.327807 & 2.614461 \\
\hline 22 & 1 & 0 & -0.533015 & -2.313700 & 2.770524 \\
\hline 23 & 1 & 0 & 2.755702 & 0.551041 & 0.173360 \\
\hline 24 & 1 & 0 & 0.850499 & -1.291257 & 0.157396 \\
\hline 25 & 15 & 0 & 0.177859 & -0.303266 & -2.291696 \\
\hline 26 & 1 & 0 & 1.405131 & -0.673805 & -2.874286 \\
\hline 27 & 1 & 0 & -0.631718 & -1.347374 & -2.778887 \\
\hline 28 & 1 & 0 & -0.201384 & 0.725509 & -3.177428 \\
\hline
\end{tabular}

Basis set A

Selected geometrical parameters : Pd-H1 1.555, Pd-H2 2.745, Pd-S1 2.458, Pd-P1 2.302, Pd-P4 2.342, S2-H2 1.359, S1-Pd-H1 178.9, P1-Pd-P4 170.6.

Frequencies:

\begin{tabular}{|c|c|c|c|c|}
\hline & & 1 & 2 & 3 \\
\hline & & A & A & A \\
\hline Frequencies & -- & 27.0892 & 34.3348 & 47.4943 \\
\hline Red. masses & -- & 9.7690 & 8.6772 & 2.4800 \\
\hline Frc consts & -- & 0.0042 & 0.0060 & 0.0033 \\
\hline IR Inten & -- & 0.2395 & 0.1191 & 2.5503 \\
\hline
\end{tabular}


[Pd(SPS) $\left.\left(\mathrm{PH}_{3}\right)(\mathrm{H})_{2}\right]^{+}$molecular (VIId)

13

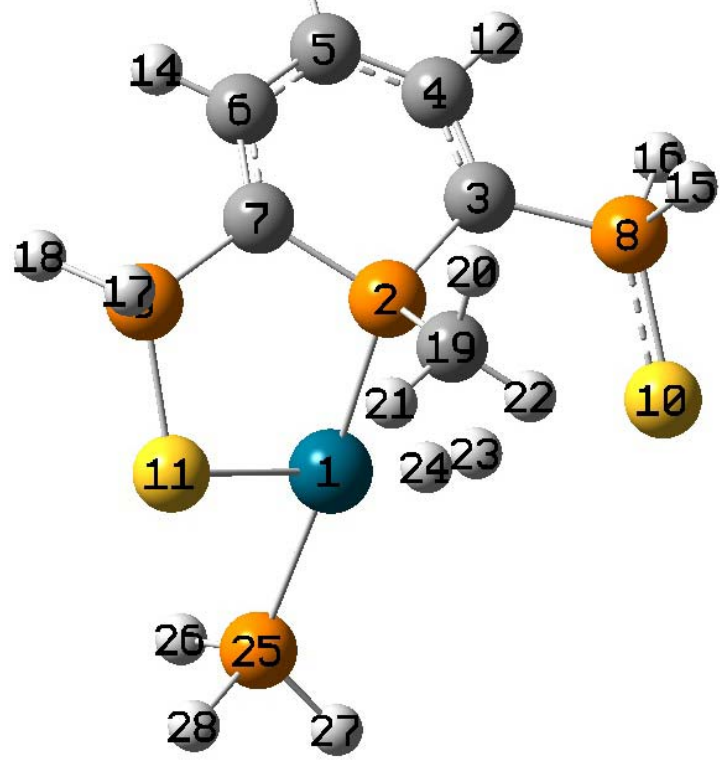

Optimized geometry:

\begin{tabular}{|c|c|c|c|c|c|}
\hline \multirow{2}{*}{$\begin{array}{l}\text { Center } \\
\text { Number }\end{array}$} & \multirow{2}{*}{$\begin{array}{l}\text { Atomic } \\
\text { Number }\end{array}$} & \multirow{2}{*}{$\begin{array}{l}\text { Atomic } \\
\text { Type }\end{array}$} & \multicolumn{3}{|c|}{ Coordinates (Angstroms) } \\
\hline & & & $\mathrm{x}$ & Y & Z \\
\hline 1 & 46 & 0 & -.157156 & -.036496 & -1.354063 \\
\hline 2 & 15 & 0 & -.114253 & -.132030 & .998435 \\
\hline 3 & 6 & 0 & 1.403973 & -.152107 & 1.909912 \\
\hline 4 & 6 & 0 & 1.827030 & .968822 & 2.618405 \\
\hline 5 & 6 & 0 & 1.094638 & 2.145929 & 2.831695 \\
\hline 6 & 6 & 0 & -.186154 & 2.323362 & 2.321593 \\
\hline 7 & 6 & 0 & -.868418 & 1.379018 & 1.545834 \\
\hline 8 & 15 & 0 & 2.470960 & -1.606963 & 1.824597 \\
\hline 9 & 15 & 0 & -2.360709 & 1.748333 & .685870 \\
\hline 10 & 16 & 0 & 2.421549 & -2.632341 & .141269 \\
\hline 11 & 16 & 0 & -2.404297 & .792708 & -1.121828 \\
\hline 12 & 1 & 0 & 2.815699 & .923985 & 3.077610 \\
\hline 13 & 1 & 0 & 1.546145 & 2.944599 & 3.409426 \\
\hline 14 & 1 & 0 & -.681394 & 3.272778 & 2.524139 \\
\hline 15 & 1 & 0 & 2.156562 & -2.393602 & 2.957629 \\
\hline 16 & 1 & 0 & 3.722738 & -1.074005 & 2.208515 \\
\hline 17 & 1 & 0 & -3.522831 & 1.370440 & 1.386041 \\
\hline 18 & 1 & 0 & -2.491210 & 3.152276 & .633922 \\
\hline 19 & 6 & 0 & -1.098916 & -1.623949 & 1.411565 \\
\hline 20 & 1 & 0 & -1.218951 & -1.692391 & 2.497817 \\
\hline 21 & 1 & 0 & -2.077975 & -1.567156 & .926860 \\
\hline 22 & 1 & 0 & -.578484 & -2.511513 & 1.036287 \\
\hline 23 & 1 & 0 & 1.428596 & -.986657 & -1.112112 \\
\hline 24 & 1 & 0 & 1.525247 & -.357097 & -1.650079 \\
\hline 25 & 15 & 0 & -.364034 & .141540 & -3.786716 \\
\hline 26 & 1 & 0 & .032761 & 1.358973 & -4.375424 \\
\hline 27 & 1 & 0 & .340712 & -.756426 & -4.613552 \\
\hline 28 & 1 & 0 & -1.649231 & .022191 & -4.351516 \\
\hline
\end{tabular}

Basis set $A$

Selected geometrical parameters : H1-H2 0.834, Pd-H1 1.864, Pd-H2 1.738, Pd-S1 2.406, Pd-S2 3.953, Pd-P1 2.355, Pd-P4 2.448, P1-Pd-P4 175.8, S1-Pd-H1 163.2, S1-Pd-H2 169.7 .

Frequencies:

\begin{tabular}{|c|c|c|c|c|}
\hline & & 1 & 2 & 3 \\
\hline & & A & A & A \\
\hline Frequencies & -- & 34.7000 & 42.7386 & 49.8957 \\
\hline Red. masses & -- & 2.1221 & 2.6547 & 2.9612 \\
\hline Frc consts & -- & .0015 & .0029 & .0043 \\
\hline IR Inten & -- & 2.9340 & 1.8298 & 1.7184 \\
\hline
\end{tabular}


[Pt (SPS) (Cl)]

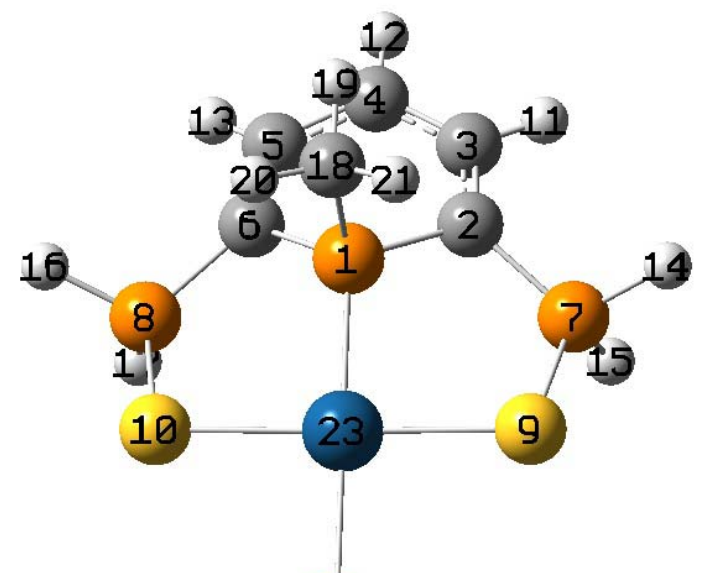

22

Optimized geometry:

\begin{tabular}{|c|c|c|c|c|c|}
\hline \multirow{2}{*}{$\begin{array}{l}\text { Center } \\
\text { Number }\end{array}$} & \multirow{2}{*}{$\begin{array}{l}\text { Atomic } \\
\text { Number }\end{array}$} & \multirow{2}{*}{$\begin{array}{c}\text { Atomic } \\
\text { Type }\end{array}$} & \multicolumn{3}{|c|}{ Coordinates (Angstroms) } \\
\hline & & & $\mathrm{X}$ & Y & Z \\
\hline 1 & 15 & 0 & 0.865035 & 0.940632 & 0.308479 \\
\hline 2 & 6 & 0 & 0.858258 & 0.928391 & 2.099213 \\
\hline 3 & 6 & 0 & 2.069716 & 0.959683 & 2.786337 \\
\hline 4 & 6 & 0 & 3.329557 & 0.799557 & 2.185897 \\
\hline 5 & 6 & 0 & 3.478532 & 0.287167 & 0.886424 \\
\hline 6 & 6 & 0 & 2.444238 & 0.171303 & -0.039623 \\
\hline 7 & 15 & 0 & -0.728416 & 0.552417 & 2.770739 \\
\hline 8 & 15 & 0 & 2.375870 & -0.929454 & -1.415667 \\
\hline 9 & 16 & 0 & -2.163581 & 0.410391 & 1.324023 \\
\hline 10 & 16 & 0 & 0.557545 & -0.888579 & -2.345658 \\
\hline 11 & 1 & 0 & 2.056988 & 1.035400 & 3.875430 \\
\hline 12 & 1 & 0 & 4.211838 & 0.876494 & 2.812889 \\
\hline 13 & 1 & 0 & 4.462080 & -0.112701 & 0.631950 \\
\hline 14 & 1 & 0 & -1.137994 & 1.516459 & 3.719226 \\
\hline 15 & 1 & 0 & -0.656649 & -0.595921 & 3.597551 \\
\hline 16 & 1 & 0 & 3.376988 & -0.638825 & -2.369636 \\
\hline 17 & 1 & 0 & 2.772656 & -2.232941 & -1.027166 \\
\hline 18 & 6 & 0 & 0.969924 & 2.686434 & -0.231709 \\
\hline 19 & 1 & 0 & 1.831437 & 3.177977 & 0.233123 \\
\hline 20 & 1 & 0 & 1.068892 & 2.716120 & -1.322003 \\
\hline 21 & 1 & 0 & 0.047969 & 3.203471 & 0.054802 \\
\hline 22 & 17 & 0 & -2.631080 & -1.503401 & -1.418824 \\
\hline 23 & 78 & 0 & -0.823051 & -0.245972 & -0.523237 \\
\hline
\end{tabular}

Basis set $A$

Frequencies:

Frequencies -- $\quad 31.8393$

Red. masses -- 6.8233

Frc consts -- 0.0041

IR Inten -- $\quad 0.0020$

2

50.2598

4.9542

0.0074

4.5995
3

9.1227

9.2500

0.0191

1.8365 
$\left[\mathrm{Pt}(\mathrm{SPS})(\mathrm{Cl})(\mathrm{H})_{2}\right]$ syn-SS (Ie)

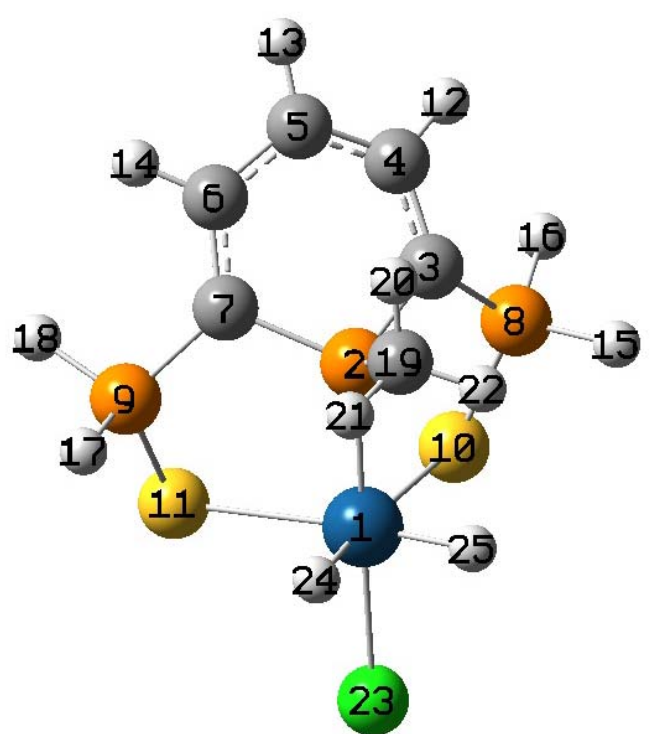

\begin{tabular}{|c|c|c|c|c|c|}
\hline \multirow{2}{*}{$\begin{array}{l}\text { Center } \\
\text { Number }\end{array}$} & \multirow{2}{*}{$\begin{array}{l}\text { Atomic } \\
\text { Number }\end{array}$} & \multirow{2}{*}{$\begin{array}{c}\text { Atomic } \\
\text { Type }\end{array}$} & \multicolumn{3}{|c|}{ Coordinates (Angstroms) } \\
\hline & & & $\mathrm{x}$ & $\mathrm{Y}$ & Z \\
\hline 1 & 78 & 0 & -0.298254 & -0.464549 & -0.969513 \\
\hline 2 & 15 & 0 & -0.330283 & -0.514756 & 1.315702 \\
\hline 3 & 6 & 0 & 1.317650 & -0.508424 & 2.002005 \\
\hline 4 & 6 & 0 & 1.519355 & 0.101274 & 3.240855 \\
\hline 5 & 6 & 0 & 0.572442 & 0.891641 & 3.909843 \\
\hline 6 & 6 & 0 & -0.540639 & 1.423256 & 3.241024 \\
\hline 7 & 6 & 0 & -1.011062 & 0.985907 & 2.002236 \\
\hline 8 & 15 & 0 & 2.594791 & -0.827024 & 0.823514 \\
\hline 9 & 15 & 0 & -1.832679 & 2.014366 & 0.823834 \\
\hline 10 & 16 & 0 & 2.237921 & -0.008575 & -0.980794 \\
\hline 11 & 16 & 0 & -0.939679 & 2.031106 & -0.980314 \\
\hline 12 & 1 & 0 & 2.511648 & 0.043021 & 3.690977 \\
\hline 13 & 1 & 0 & 0.833479 & 1.298267 & 4.881332 \\
\hline 14 & 1 & 0 & -1.006967 & 2.301011 & 3.691283 \\
\hline 15 & 1 & 0 & 2.845043 & -2.216849 & 0.725031 \\
\hline 16 & 1 & 0 & 3.798713 & -0.380786 & 1.410434 \\
\hline 17 & 1 & 0 & -3.200352 & 1.662745 & 0.725075 \\
\hline 18 & 1 & 0 & -1.928723 & 3.294648 & 1.410934 \\
\hline 19 & 6 & 0 & -1.238856 & -1.930819 & 2.033823 \\
\hline 20 & 1 & 0 & -1.203118 & -1.875256 & 3.126673 \\
\hline 21 & 1 & 0 & -2.280471 & -1.903752 & 1.697772 \\
\hline 22 & 1 & 0 & -0.780226 & -2.866385 & 1.697661 \\
\hline 23 & 17 & 0 & -0.321869 & -0.501133 & -3.355199 \\
\hline 24 & 1 & 0 & -1.793549 & -0.845968 & -1.021621 \\
\hline 25 & 1 & 0 & -0.022532 & -1.982875 & -1.021896 \\
\hline
\end{tabular}

Basis set $A$

Selected geometrical parameters : H-H 2.105, Pd-H1 1.544, Pt-S1 2.577, Pt-P1 2.286, Pt-Cl 2.386, S-Pt-S 94.2, P1-Pt-Cl 177.5.

Frequencies:

\begin{tabular}{llr} 
& & \multicolumn{1}{c}{ A } \\
& & \multicolumn{1}{c}{ A.3937 } \\
Frequencies -- & 51.39101 \\
Red. masses -- & 7.510 \\
Frc consts -- & 0.0117 \\
IR Inten & -- & 0.0465
\end{tabular}

2
A
64.6446
5.4281
0.0134
3.3215

3
$A$
92.3013
1.1224
0.0558
1.1708


[Pt (SPS) $\left.(\mathrm{Cl})(\mathrm{H})_{2}\right] \quad M H \ldots S H \quad$ (VIe)

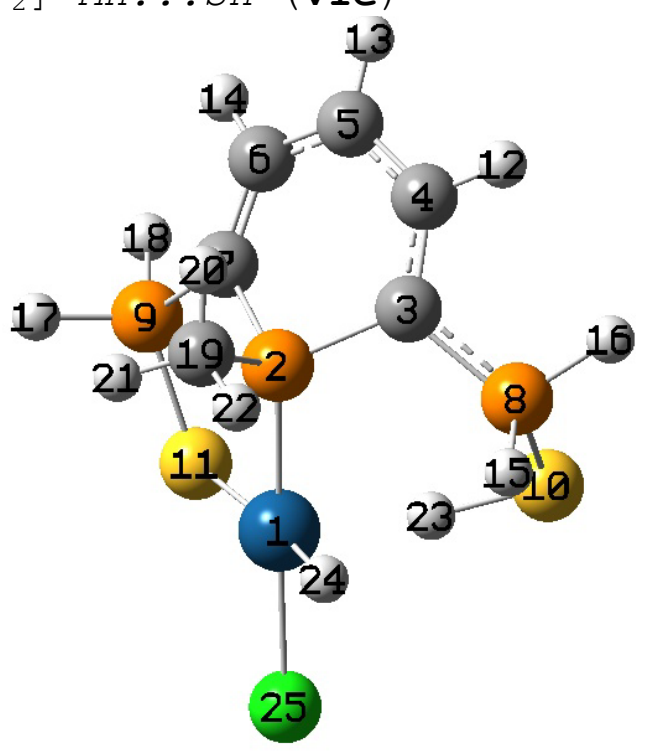

Optimized geometry:

\begin{tabular}{|c|c|c|c|c|c|}
\hline \multirow{2}{*}{$\begin{array}{l}\text { Center } \\
\text { Number }\end{array}$} & \multirow{2}{*}{$\begin{array}{l}\text { Atomic } \\
\text { Number }\end{array}$} & \multirow{2}{*}{$\begin{array}{c}\text { Atomic } \\
\text { Type }\end{array}$} & \multicolumn{3}{|c|}{ Coordinates (Angstroms) } \\
\hline & & & $\mathrm{X}$ & Y & Z \\
\hline 1 & 78 & 0 & -.351430 & -.462118 & -1.001797 \\
\hline 2 & 15 & 0 & -.339902 & -.493257 & 1.243164 \\
\hline 3 & 6 & 0 & 1.290744 & -.496941 & 1.999026 \\
\hline 4 & 6 & 0 & 1.493932 & .148968 & 3.229776 \\
\hline 5 & 6 & 0 & .564885 & .976019 & 3.856360 \\
\hline 6 & 6 & 0 & -.551172 & 1.490853 & 3.163420 \\
\hline 7 & 6 & 0 & -1.012449 & 1.021360 & 1.944351 \\
\hline 8 & 15 & 0 & 2.630885 & -.939836 & .972091 \\
\hline 9 & 15 & 0 & -2.011103 & 1.964278 & .825889 \\
\hline 10 & 16 & 0 & 2.967944 & .198458 & -.706703 \\
\hline 11 & 16 & 0 & -1.428837 & 1.814373 & -1.097448 \\
\hline 12 & 1 & 0 & 2.470906 & .053947 & 3.707715 \\
\hline 13 & 1 & 0 & .817203 & 1.405263 & 4.820360 \\
\hline 14 & 1 & 0 & -1.018233 & 2.378931 & 3.592714 \\
\hline 15 & 1 & 0 & 2.544280 & -2.286921 & .571360 \\
\hline 16 & 1 & 0 & 3.799918 & -.902277 & 1.751535 \\
\hline 17 & 1 & 0 & -3.376231 & 1.622947 & .984389 \\
\hline 18 & 1 & 0 & -2.022943 & 3.288608 & 1.317738 \\
\hline 19 & 6 & 0 & -1.253643 & -1.879678 & 2.025137 \\
\hline 20 & 1 & 0 & -1.211016 & -1.793958 & 3.115833 \\
\hline 21 & 1 & 0 & -2.297378 & -1.861406 & 1.693752 \\
\hline 22 & 1 & 0 & -.804425 & -2.827598 & 1.711219 \\
\hline 23 & 1 & 0 & 1.524241 & -.004603 & -1.018062 \\
\hline 24 & 1 & 0 & .233097 & -1.900579 & -1.028601 \\
\hline 25 & 17 & 0 & -.309738 & -.521394 & -3.361113 \\
\hline
\end{tabular}

Basis set $A$

Selected geometrical parameters : H1 1.553, Pt-H2 1.931, Pt-S1 2.520, Pt-P1 2.245, Pt-Cl 2.360, S2-H2 1.491, S1-Pt-H1 175.5, P1-Pt-Cl 177.4.

Frequencies:

\begin{tabular}{|c|c|c|c|c|}
\hline 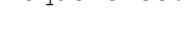 & & 1 & 2 & 3 \\
\hline & & A & A & A \\
\hline Frequencies & -- & 45.2776 & 53.7356 & 61.7255 \\
\hline Red. masses & -- & 10.0893 & 8.2679 & 10.5172 \\
\hline Frc consts & -- & .0122 & .0141 & .0236 \\
\hline IR Inten & -- & 1.3441 & .1817 & 1.3619 \\
\hline
\end{tabular}


[PtSPS) (Cl) ( $\mathrm{H}_{2}$ ] molecular (VIIe)

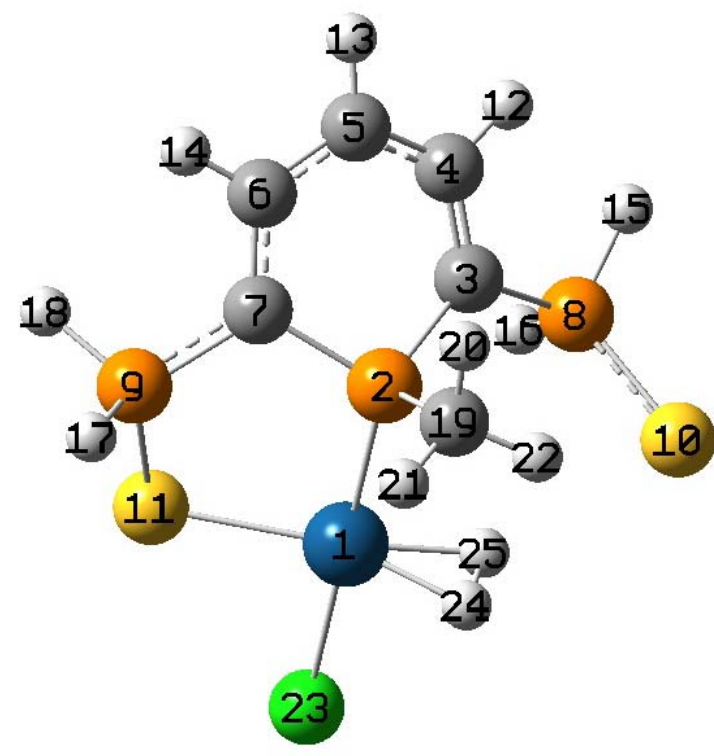

Optimized geometry:

\begin{tabular}{|c|c|c|c|c|c|}
\hline \multirow{2}{*}{$\begin{array}{l}\text { Center } \\
\text { Number }\end{array}$} & \multirow{2}{*}{$\begin{array}{l}\text { Atomic } \\
\text { Number }\end{array}$} & \multirow{2}{*}{$\begin{array}{l}\text { Atomic } \\
\text { Type }\end{array}$} & \multicolumn{3}{|c|}{ Coordinates (Angstroms) } \\
\hline & & & $\mathrm{X}$ & Y & Z \\
\hline 1 & 78 & 0 & -.270709 & -.199839 & -1.139382 \\
\hline 2 & 15 & 0 & -.309297 & -.206064 & 1.156442 \\
\hline 3 & 6 & 0 & 1.254589 & -.192106 & 2.032752 \\
\hline 4 & 6 & 0 & 1.320479 & .440305 & 3.260271 \\
\hline 5 & 6 & 0 & .334516 & 1.288300 & 3.817095 \\
\hline 6 & 6 & 0 & -.724489 & 1.766953 & 3.061102 \\
\hline 7 & 6 & 0 & -1.054892 & 1.290388 & 1.777474 \\
\hline 8 & 15 & 0 & 2.693264 & -.989089 & 1.323195 \\
\hline 9 & 15 & 0 & -1.974154 & 2.186052 & .602986 \\
\hline 10 & 16 & 0 & 2.481153 & -2.791240 & .560253 \\
\hline 11 & 16 & 0 & -1.257902 & 1.931788 & -1.307631 \\
\hline 12 & 1 & 0 & 2.246380 & .337518 & 3.829915 \\
\hline 13 & 1 & 0 & .510137 & 1.708379 & 4.801775 \\
\hline 14 & 1 & 0 & -1.294096 & 2.602668 & 3.468967 \\
\hline 15 & 1 & 0 & 3.621543 & -.863753 & 2.385401 \\
\hline 16 & 1 & 0 & 3.226464 & -.061381 & .396464 \\
\hline 17 & 1 & 0 & -3.361179 & 1.904343 & .601996 \\
\hline 18 & 1 & 0 & -1.988364 & 3.550959 & .963399 \\
\hline 19 & 6 & 0 & -1.231856 & -1.659824 & 1.788824 \\
\hline 20 & 1 & 0 & -1.314249 & -1.590879 & 2.878636 \\
\hline 21 & 1 & 0 & -2.231071 & -1.694835 & 1.341919 \\
\hline 22 & 1 & 0 & -.679805 & -2.569109 & 1.526559 \\
\hline 23 & 17 & 0 & -.206796 & -.185966 & -3.512363 \\
\hline 24 & 1 & 0 & -.025118 & -1.817896 & -1.270931 \\
\hline 25 & 1 & 0 & .810917 & -1.473541 & -1.048549 \\
\hline
\end{tabular}

Basis set $A$

Selected geometrical parameters : H1-H2 0.931, Pd-H1 1.642, Pd-H2 1.673, Pd-S1 2.355, Pd-S2 4.145, Pd-P1 2.296, Pd-Cl 2.374, P1-Pd-Cl 179.4, S1-Pd-H1 161.6, S1-Pd-H2 164.5.

Frequencies:

\begin{tabular}{lrrr} 
& 1 & \multicolumn{1}{c}{2} & \multicolumn{1}{c}{3} \\
Frequencies -- & 31.4604 & \multicolumn{1}{c}{ A } & A \\
Red. masses -- & 13.1651 & 9.8732 & 61.2860 \\
Frc consts -- & .0077 & .0151 & 7.8847 \\
IR Inten -- & .8100 & 1.0605 & .0174 \\
\end{tabular}


$\left[\mathrm{Pt}(\mathrm{SPS})\left(\mathrm{PH}_{3}\right)\right]^{+}$

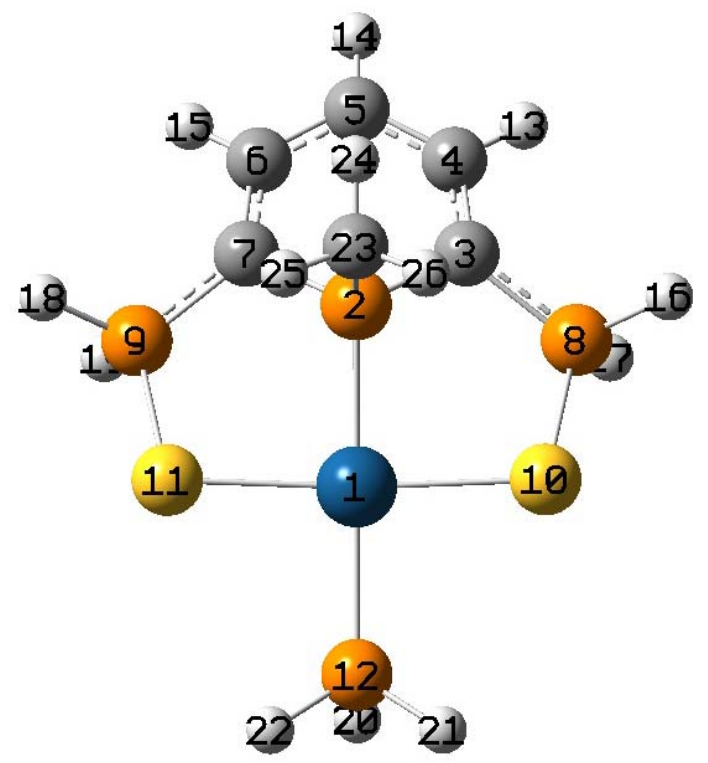

Optimized geometry:

\begin{tabular}{|c|c|c|c|c|c|}
\hline \multirow{2}{*}{$\begin{array}{l}\text { Center } \\
\text { Number }\end{array}$} & \multirow{2}{*}{$\begin{array}{l}\text { Atomic } \\
\text { Number }\end{array}$} & \multirow{2}{*}{$\begin{array}{l}\text { Atomic } \\
\text { Type }\end{array}$} & \multicolumn{3}{|c|}{ Coordinates (Angstroms) } \\
\hline & & & $\mathrm{x}$ & Y & Z \\
\hline 1 & 78 & 0 & -0.177672 & -0.264151 & -0.957257 \\
\hline 2 & 15 & 0 & -0.175196 & -0.260571 & 1.324511 \\
\hline 3 & 6 & 0 & 1.494812 & -0.257212 & 1.954727 \\
\hline 4 & 6 & 0 & 1.787310 & 0.458916 & 3.118041 \\
\hline 5 & 6 & 0 & 0.910294 & 1.353407 & 3.745773 \\
\hline 6 & 6 & 0 & -0.248951 & 1.828295 & 3.118118 \\
\hline 7 & 6 & 0 & -0.801928 & 1.287341 & 1.954812 \\
\hline 8 & 15 & 0 & 2.668994 & -0.983434 & 0.867686 \\
\hline 9 & 15 & 0 & -1.917458 & 2.100926 & 0.867879 \\
\hline 10 & 16 & 0 & 1.773950 & -1.624304 & -0.869967 \\
\hline 11 & 16 & 0 & -2.173374 & 1.030445 & -0.869891 \\
\hline 12 & 15 & 0 & -0.137723 & -0.204667 & -3.315211 \\
\hline 13 & 1 & 0 & 2.788841 & 0.381589 & 3.542813 \\
\hline 14 & 1 & 0 & 1.233335 & 1.833717 & 4.662668 \\
\hline 15 & 1 & 0 & -0.698294 & 2.726675 & 3.542948 \\
\hline 16 & 1 & 0 & 3.327182 & -2.105149 & 1.412361 \\
\hline 17 & 1 & 0 & 3.754340 & -0.109184 & 0.633527 \\
\hline 18 & 1 & 0 & -3.204583 & 2.287280 & 1.412591 \\
\hline 19 & 1 & 0 & -1.517232 & 3.435907 & 0.633864 \\
\hline 20 & 1 & 0 & 0.580732 & 0.863330 & -3.885380 \\
\hline 21 & 1 & 0 & 0.438117 & -1.291546 & -4.000751 \\
\hline 22 & 1 & 0 & -1.361509 & -0.080888 & -4.000696 \\
\hline 23 & 6 & 0 & -1.106328 & -1.645207 & 2.065978 \\
\hline 24 & 1 & 0 & -1.061351 & -1.578364 & 3.158387 \\
\hline 25 & 1 & 0 & -2.149966 & -1.600156 & 1.736900 \\
\hline 26 & 1 & 0 & -0.670964 & -2.594758 & 1.736867 \\
\hline
\end{tabular}

Basis set A

Frequencies:

\begin{tabular}{llr} 
& & \multicolumn{1}{c}{ A } \\
Frequencies -- & 32.7465 \\
Red. masses -- & 4.8527 \\
Frc consts -- & 0.0031 \\
IR Inten & -- & 0.3053
\end{tabular}

2
A
40.8684
4.4499
0.0044
2.2809

3

A

55.7196

5.3032

0.0097

8.7254 
$\left[\mathrm{Pt}(\mathrm{SPS})\left(\mathrm{PH}_{3}\right)(\mathrm{H})_{2}\right]^{+}$syn-SS (If)

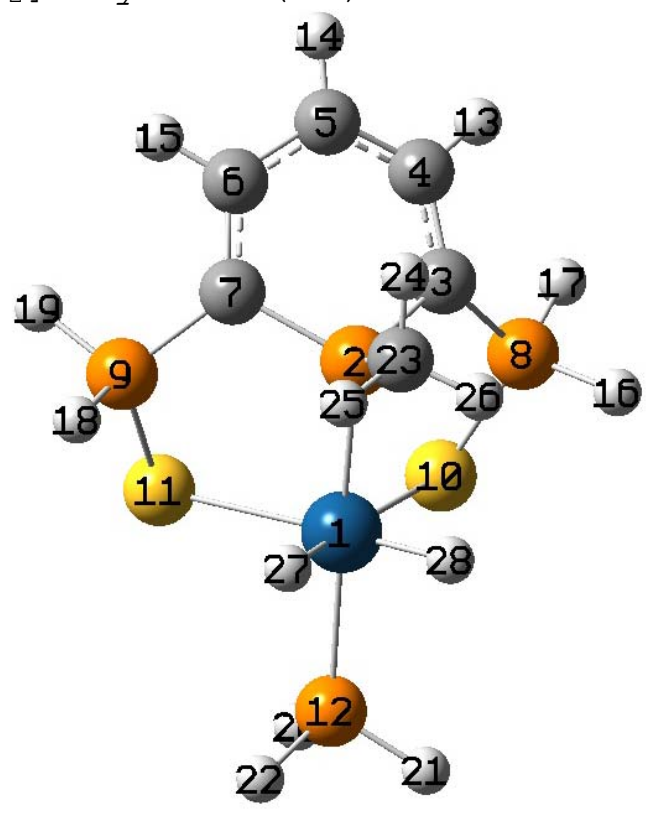

Optimized geometry:

\begin{tabular}{|c|c|c|c|c|c|}
\hline \multirow{2}{*}{$\begin{array}{l}\text { Center } \\
\text { Number }\end{array}$} & \multirow{2}{*}{$\begin{array}{l}\text { Atomic } \\
\text { Number }\end{array}$} & \multirow{2}{*}{$\begin{array}{l}\text { Atomic } \\
\text { Type }\end{array}$} & \multicolumn{3}{|c|}{ Coordinates (Angstroms) } \\
\hline & & & $\mathrm{X}$ & Y & Z \\
\hline 1 & 78 & 0 & -0.302190 & -0.461123 & -0.938614 \\
\hline 2 & 15 & 0 & -0.324855 & -0.495897 & 1.387858 \\
\hline 3 & 6 & 0 & 1.338064 & -0.488495 & 2.022805 \\
\hline 4 & 6 & 0 & 1.580609 & 0.180333 & 3.227897 \\
\hline 5 & 6 & 0 & 0.662524 & 1.011178 & 3.883044 \\
\hline 6 & 6 & 0 & -0.465814 & 1.521118 & 3.227890 \\
\hline 7 & 6 & 0 & -0.982143 & 1.031619 & 2.022828 \\
\hline 8 & 15 & 0 & 2.576018 & -0.876961 & 0.833629 \\
\hline 9 & 15 & 0 & -1.832681 & 2.011381 & 0.833611 \\
\hline 10 & 16 & 0 & 2.194293 & -0.026690 & -0.975700 \\
\hline 11 & 16 & 0 & -0.900563 & 2.001223 & -0.975645 \\
\hline 12 & 15 & 0 & -0.366442 & -0.559369 & -3.305816 \\
\hline 13 & 1 & 0 & 2.581124 & 0.123709 & 3.657240 \\
\hline 14 & 1 & 0 & 0.951612 & 1.452401 & 4.830462 \\
\hline 15 & 1 & 0 & -0.917239 & 2.415816 & 3.657196 \\
\hline 16 & 1 & 0 & 2.761228 & -2.269956 & 0.698275 \\
\hline 17 & 1 & 0 & 3.817777 & -0.454154 & 1.343767 \\
\hline 18 & 1 & 0 & -3.183647 & 1.624634 & 0.698091 \\
\hline 19 & 1 & 0 & -1.941227 & 3.318610 & 1.343851 \\
\hline 20 & 1 & 0 & 0.303047 & 0.459712 & -4.006476 \\
\hline 21 & 1 & 0 & 0.180925 & -1.709991 & -3.897440 \\
\hline 22 & 1 & 0 & -1.639710 & -0.514109 & -3.897679 \\
\hline 23 & 6 & 0 & -1.246471 & -1.902621 & 2.093704 \\
\hline 24 & 1 & 0 & -1.207714 & -1.843541 & 3.186342 \\
\hline 25 & 1 & 0 & -2.289523 & -1.866374 & 1.763431 \\
\hline 26 & 1 & 0 & -0.796735 & -2.844409 & 1.763366 \\
\hline 27 & 1 & 0 & -1.818024 & -0.826565 & -0.907647 \\
\hline 28 & 1 & 0 & -0.031998 & -1.996797 & -0.907608 \\
\hline
\end{tabular}

Basis set A

Selected geometrical parameters : H-H 2.135, Pt-H1 1.560, Pt-S1 2.534, Pt-P1 2.327, Pt-P4 2.370, S-Pt-S 93.8, P1-Pt-P4 176.1.

Frequencies:

\begin{tabular}{llr} 
& \multicolumn{1}{c}{1} \\
& \multicolumn{1}{c}{ A } \\
Frequencies -- & 39.9686 \\
Red. masses -- & 1.2874 \\
Frc consts -- & 0.0012 \\
IR Inten -- & 0.3523
\end{tabular}

\section{2}

57.6059

2. 9409

0.0057

0.7629

\section{3}

63.9244

4.8934

0.0118

0.1846 
$\left[\mathrm{Pt}(\mathrm{SPS})\left(\mathrm{PH}_{3}\right)(\mathrm{H})_{2}\right]^{+} \mathrm{MH} \ldots \mathrm{SH} \quad(\mathbf{V I f})$

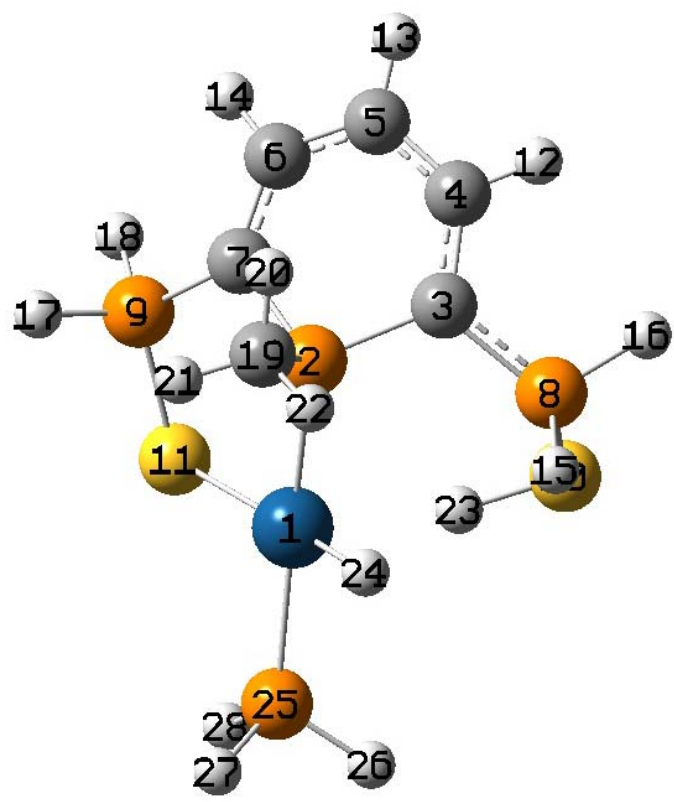

Optimized geometry:

\begin{tabular}{|c|c|c|c|c|c|}
\hline \multirow{2}{*}{$\begin{array}{l}\text { Center } \\
\text { Number }\end{array}$} & \multirow{2}{*}{$\begin{array}{l}\text { Atomic } \\
\text { Number }\end{array}$} & \multirow{2}{*}{$\begin{array}{l}\text { Atomic } \\
\text { Type }\end{array}$} & \multicolumn{3}{|c|}{ Coordinates (Angstroms) } \\
\hline & & & $\mathrm{x}$ & Y & Z \\
\hline 1 & 78 & 0 & -0.311927 & -0.424215 & -1.032682 \\
\hline 2 & 15 & 0 & -0.384998 & -0.469720 & 1.268579 \\
\hline 3 & 6 & 0 & 1.202127 & -0.465954 & 2.104030 \\
\hline 4 & 6 & 0 & 1.336156 & 0.207674 & 3.336346 \\
\hline 5 & 6 & 0 & 0.380446 & 1.047470 & 3.893132 \\
\hline 6 & 6 & 0 & -0.705626 & 1.541078 & 3.141319 \\
\hline 7 & 6 & 0 & -1.104846 & 1.046557 & 1.908566 \\
\hline 8 & 15 & 0 & 2.599394 & -0.978081 & 1.209839 \\
\hline 9 & 15 & 0 & -2.095168 & 1.944736 & 0.756547 \\
\hline 10 & 16 & 0 & 3.194537 & 0.174350 & -0.425284 \\
\hline 11 & 16 & 0 & -1.409905 & 1.775235 & -1.152403 \\
\hline 12 & 1 & 0 & 2.278702 & 0.107676 & 3.876032 \\
\hline 13 & 1 & 0 & 0.578055 & 1.488515 & 4.863871 \\
\hline 14 & 1 & 0 & -1.205359 & 2.425127 & 3.538422 \\
\hline 15 & 1 & 0 & 2.477983 & -2.299950 & 0.748322 \\
\hline 16 & 1 & 0 & 3.712098 & -0.995230 & 2.064437 \\
\hline 17 & 1 & 0 & -3.452792 & 1.563204 & 0.825495 \\
\hline 18 & 1 & 0 & -2.152050 & 3.287036 & 1.179420 \\
\hline 19 & 6 & 0 & -1.344848 & -1.873219 & 1.947571 \\
\hline 20 & 1 & 0 & -1.389340 & -1.801290 & 3.038963 \\
\hline 21 & 1 & 0 & -2.360105 & -1.859166 & 1.537280 \\
\hline 22 & 1 & 0 & -0.864738 & -2.814675 & 1.661362 \\
\hline 23 & 1 & 0 & 1.890952 & 0.087711 & -0.911985 \\
\hline 24 & 1 & 0 & 0.359227 & -1.842978 & -0.971992 \\
\hline 25 & 15 & 0 & -0.248791 & -0.647071 & -3.346453 \\
\hline 26 & 1 & 0 & 0.965653 & -1.038807 & -3.939791 \\
\hline 27 & 1 & 0 & -1.110367 & -1.630328 & -3.867833 \\
\hline 28 & 1 & 0 & -0.588729 & 0.448994 & -4.163236 \\
\hline
\end{tabular}

Basis set A

Selected geometrical parameters : Pt-H1 1.571, Pt-H2 2.265, Pt-S1 2.461, Pt-P1 2.303, Pt-P4 2.325, S2-H2 1.394, S1-Pt-H1 178.7, P1-Pt-P4 173.4.

Frequencies:

\begin{tabular}{|c|c|c|c|c|}
\hline & & 1 & 2 & 3 \\
\hline & & A & A & A \\
\hline Frequencies & -- & 38.7360 & 49.7328 & 58.1573 \\
\hline Red. masses & -- & 8.9233 & 3.2150 & 7.3026 \\
\hline Frc consts & -- & 0.0079 & 0.0047 & 0.0146 \\
\hline IR Inten & -- & 0.3220 & 2.5396 & 0.4853 \\
\hline
\end{tabular}


Table 1: Crystal data of compound 4

\begin{tabular}{|c|c|}
\hline Compound & irspsh2 \\
\hline Molecular formula & $\mathrm{C}_{60} \mathrm{H}_{51} \mathrm{IrP}_{4} \mathrm{~S}_{2}, 2\left(\mathrm{CH}_{2} \mathrm{Cl}_{2}\right)$ \\
\hline Molecular weight & 1322.06 \\
\hline Crystal habit & orange plate \\
\hline Crystal dimensions $(\mathrm{mm})$ & $0.21 \times 0.20 \times 0.12$ \\
\hline Crystal system & triclinic \\
\hline Space group & Pbar1 \\
\hline $\mathrm{a}(\AA)$ & $11.3410(10)$ \\
\hline $\mathrm{b}(\AA)$ & $14.4510(10)$ \\
\hline$c(\AA)$ & $18.6570(10)$ \\
\hline$\alpha\left(^{\circ}\right)$ & $85.2690(10)$ \\
\hline$\beta\left(^{\circ}\right)$ & $77.1100(10)$ \\
\hline$\gamma\left({ }^{\circ}\right)$ & $73.9350(10)$ \\
\hline $\mathrm{V}\left(\AA^{3}\right)$ & $2863.5(4)$ \\
\hline $\mathrm{Z}$ & 2 \\
\hline$d\left(g-\mathrm{cm}^{-3}\right)$ & 1.533 \\
\hline $\mathrm{F}(000)$ & 1328 \\
\hline$\mu\left(\mathrm{cm}^{-1}\right)$ & 2.742 \\
\hline Absorption corrections & gaussian ; $0.5967 \min , 0.7344 \max$ \\
\hline Diffractometer & KappaCCD \\
\hline X-ray source & MoK $\alpha$ \\
\hline$\lambda(\AA)$ & 0.71069 \\
\hline Monochromator & graphite \\
\hline $\mathrm{T}(\mathrm{K})$ & $150.0(1)$ \\
\hline Scan mode & phi and omega scans \\
\hline Maximum $\theta$ & 30.03 \\
\hline HKL ranges & $-1415 ;-2020 ;-2426$ \\
\hline Reflections measured & 40104 \\
\hline Unique data & 16646 \\
\hline Rint & 0.0473 \\
\hline Reflections used & 14664 \\
\hline Criterion & $\mathrm{I}>2 \sigma \mathrm{I})$ \\
\hline Refinement type & Fsqd \\
\hline Hydrogen atoms & mixed \\
\hline Parameters refined & 673 \\
\hline Reflections / parameter & 21 \\
\hline wR2 & 0.0664 \\
\hline R1 & 0.0281 \\
\hline Weights $a, b$ & $0.0289 ; 0.0000$ \\
\hline GoF & 1.018 \\
\hline difference peak / hole $\left(\mathrm{e} \AA^{-3}\right)$ & $1.216(0.115) /-1.185(0.115)$ \\
\hline
\end{tabular}


Table 2. Atomic Coordinates (A x 10^4) and equivalent isotropic displacement parameters $\left(A^{\wedge} 2 \times 10^{\wedge} 3\right)$ for 4

\begin{tabular}{|c|c|c|c|c|}
\hline atom & $\mathrm{x}$ & $\mathrm{y}$ & $z$ & $\mathrm{U}(\mathrm{eq})$ \\
\hline $\operatorname{Ir}(1)$ & $1047(1)$ & $2571(1)$ & $7627(1)$ & $16(1)$ \\
\hline$S(1)$ & $-1236(1)$ & $3083(1)$ & $7672(1)$ & $20(1)$ \\
\hline$S(2)$ & $1448(1)$ & $3138(1)$ & $6343(1)$ & $19(1)$ \\
\hline$P(1)$ & $932(1)$ & $4055(1)$ & $7991(1)$ & $15(1)$ \\
\hline$P(2)$ & $-1726(1)$ & $4065(1)$ & $8465(1)$ & $16(1)$ \\
\hline$P(3)$ & $2314(1)$ & $4140(1)$ & $6464(1)$ & $16(1)$ \\
\hline$P(4)$ & $1261(1)$ & $1035(1)$ & $7288(1)$ & 21 (1) \\
\hline$C(1)$ & $-629(2)$ & $4763(1)$ & $8327(1)$ & $17(1)$ \\
\hline$C(2)$ & $-839(2)$ & $5765(2)$ & $8325(1)$ & $17(1)$ \\
\hline$C(3)$ & $58(2)$ & $6226(2)$ & $7916(1)$ & $18(1)$ \\
\hline$C(4)$ & $1083(2)$ & $5809(2)$ & $7357(1)$ & $17(1)$ \\
\hline$C(5)$ & $1496(2)$ & $4811(1)$ & $7255(1)$ & $16(1)$ \\
\hline$C(6)$ & $1779(2)$ & $4111(2)$ & $8704(1)$ & $23(1)$ \\
\hline$C(7)$ & $-1773(2)$ & $3435(2)$ & $9352(1)$ & $21(1)$ \\
\hline$C(8)$ & $-1126(2)$ & $3613(2)$ & $9847(1)$ & $24(1)$ \\
\hline$C(9)$ & $-1073(2)$ & $3070(2)$ & $10499(1)$ & 35 (1) \\
\hline$C(10)$ & $-1683(3)$ & $2354(2)$ & $10654(2)$ & 41 (1) \\
\hline$C(11)$ & $-2345(3)$ & $2175(2)$ & $10161(2)$ & $40(1)$ \\
\hline$C(12)$ & $-2382(2)$ & $2700(2)$ & $9510(1)$ & $30(1)$ \\
\hline$C(13)$ & $-3286(2)$ & $4789(2)$ & $8419(1)$ & $18(1)$ \\
\hline$C(14)$ & $-3422(2)$ & $5311(2)$ & $7767(1)$ & $23(1)$ \\
\hline C (15) & $-4601(2)$ & $5851(2)$ & $7678(1)$ & $27(1)$ \\
\hline$C(16)$ & $-5647(2)$ & $5866(2)$ & $8234(1)$ & $29(1)$ \\
\hline$C(17)$ & $-5514(2)$ & $5350(2)$ & $8882(1)$ & $31(1)$ \\
\hline$C(18)$ & $-4337(2)$ & $4815(2)$ & $8978(1)$ & $25(1)$ \\
\hline$C(19)$ & $-2012(2)$ & $6405(1)$ & $8752(1)$ & $17(1)$ \\
\hline$C(20)$ & $-2613(2)$ & $7278(2)$ & $8456(1)$ & $24(1)$ \\
\hline$C(21)$ & $-3674(2)$ & $7889(2)$ & $8864(1)$ & $29(1)$ \\
\hline$C(22)$ & $-4174(2)$ & $7629(2)$ & $9574(1)$ & $27(1)$ \\
\hline$C(23)$ & $-3589(2)$ & $6770(2)$ & $9879(1)$ & $29(1)$ \\
\hline$C(24)$ & $-2524(2)$ & $6164(2)$ & $9474(1)$ & $24(1)$ \\
\hline$C(25)$ & $1715(2)$ & $6483(2)$ & $6863(1)$ & $18(1)$ \\
\hline$C(26)$ & $3008(2)$ & $6227(2)$ & $6607(1)$ & $22(1)$ \\
\hline C (27) & $3610(2)$ & $6845(2)$ & $6151(1)$ & $27(1)$ \\
\hline$C(28)$ & $2918(2)$ & $7735(2)$ & 5957 (1) & $27(1)$ \\
\hline C (29) & $1636(2)$ & $8008(2)$ & $6209(1)$ & $28(1)$ \\
\hline$C(30)$ & $1035(2)$ & $7387(2)$ & $6657(1)$ & $24(1)$ \\
\hline C (31) & $2360(2)$ & $4836(2)$ & $5614(1)$ & $17(1)$ \\
\hline$C(32)$ & $3468(2)$ & $4796(2)$ & $5091(1)$ & $24(1)$ \\
\hline$C(33)$ & $3413(2)$ & $5252(2)$ & $4410(1)$ & $29(1)$ \\
\hline C (34) & $2273(2)$ & $5737(2)$ & $4240(1)$ & $29(1)$ \\
\hline$C(35)$ & $1177(2)$ & $5785(2)$ & $4759(1)$ & $29(1)$ \\
\hline$C(36)$ & $1212(2)$ & $5341(2)$ & $5443(1)$ & $25(1)$ \\
\hline$C(37)$ & $3912(2)$ & $3534(2)$ & $6557(1)$ & $19(1)$ \\
\hline$C(38)$ & $4397(2)$ & $3752(2)$ & $7125(1)$ & $21(1)$ \\
\hline C (39) & $5565(2)$ & $3230(2)$ & $7236(1)$ & $27(1)$ \\
\hline$C(40)$ & $6272(2)$ & $2484(2)$ & $6776(2)$ & 31 (1) \\
\hline$C(41)$ & $5812(2)$ & $2268(2)$ & $6199(2)$ & $34(1)$ \\
\hline$C(42)$ & $4626(2)$ & $2786(2)$ & $6094(1)$ & $27(1)$ \\
\hline$C(43)$ & $1115(2)$ & $938(2)$ & $6338(1)$ & $24(1)$ \\
\hline$C(44)$ & $2063(2)$ & $385(2)$ & $5820(1)$ & $29(1)$ \\
\hline$C(45)$ & $1928(3)$ & $375(2)$ & $5098(2)$ & $37(1)$ \\
\hline$C(46)$ & $849(3)$ & $915(2)$ & $4887(2)$ & $39(1)$ \\
\hline$C(47)$ & $-102(3)$ & $1478(2)$ & $5398(2)$ & $38(1)$ \\
\hline$C(48)$ & $37(2)$ & $1499(2)$ & $6110(2)$ & 31 (1) \\
\hline$C(49)$ & $2776(2)$ & $172(2)$ & $7315(1)$ & $27(1)$ \\
\hline$C(50)$ & $2887(3)$ & $-779(2)$ & $7547(2)$ & $43(1)$ \\
\hline$C(51)$ & $4067(4)$ & $-1396(2)$ & $7540(2)$ & $58(1)$ \\
\hline$C(52)$ & $5118(3)$ & -1095 (3) & $7295(2)$ & $60(1)$ \\
\hline$C(53)$ & $5029(3)$ & $-152(2)$ & $7056(2)$ & $52(1)$ \\
\hline$C(54)$ & $3865(2)$ & $475(2)$ & $7072(2)$ & $38(1)$ \\
\hline$C(55)$ & $132(2)$ & $422(2)$ & $7830(1)$ & $31(1)$ \\
\hline$C(56)$ & $-65(3)$ & $440(2)$ & $8596(2)$ & $44(1)$ \\
\hline$C(57)$ & $-871(3)$ & $-46(2)$ & $9038(2)$ & $53(1)$ \\
\hline$C(58)$ & $-1493(3)$ & $-537(2)$ & $8726(2)$ & $60(1)$ \\
\hline$C(59)$ & $-1328(3)$ & $-549(3)$ & $7970(2)$ & $68(1)$ \\
\hline$C(60)$ & $-520(3)$ & $-64(2)$ & $7523(2)$ & $51(1)$ \\
\hline$C(61)$ & $-2252(3)$ & $7089(2)$ & $5556(2)$ & $44(1)$ \\
\hline $\mathrm{Cl}(1)$ & $-3748(1)$ & $7892(1)$ & $5606(1)$ & $55(1)$ \\
\hline $\mathrm{Cl}(2)$ & $-1857(1)$ & $6859(1)$ & $6429(1)$ & $53(1)$ \\
\hline$C(62)$ & $3702(4)$ & $1233(2)$ & $8902(2)$ & $64(1)$ \\
\hline $\mathrm{Cl}(3)$ & $5445(2)$ & $1089(2)$ & $8644(1)$ & $69(1)$ \\
\hline $\mathrm{Cl}(4)$ & $3414(3)$ & $198(2)$ & $9287(2)$ & $73(1)$ \\
\hline $\mathrm{Cl}(3 \mathrm{~A})$ & $4883(4)$ & $1518(3)$ & $8548(2)$ & $69(1)$ \\
\hline $\mathrm{Cl}(4 \mathrm{~A})$ & $3780(6)$ & $200(5)$ & $9496(3)$ & $73(1)$ \\
\hline
\end{tabular}

$\mathrm{U}(\mathrm{eq})$ is defined as $1 / 3$ the trace of the Uij tensor. 


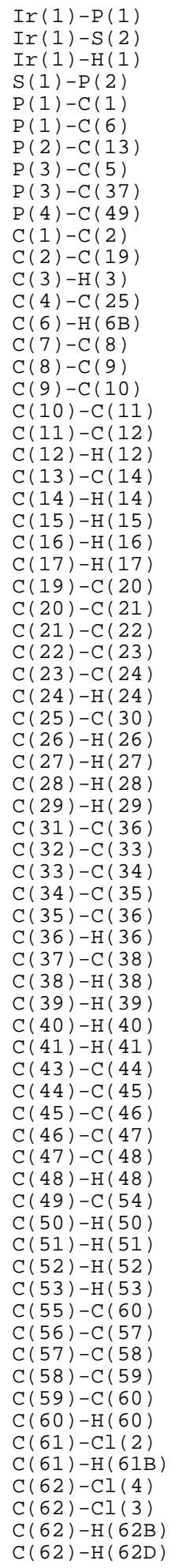

$\mathrm{P}(1)-\operatorname{Ir}(1)-\mathrm{P}(4)$

$P(4)-\operatorname{Ir}(1)-S(2)$

$\mathrm{P}(4)-\operatorname{Ir}(1)-\mathrm{S}(1)$

$\mathrm{P}(1)-\operatorname{Ir}(1)-\mathrm{H}(1)$

$\mathrm{S}(2)-\operatorname{Ir}(1)-\mathrm{H}(1)$

$\mathrm{P}(1)-\operatorname{Ir}(1)-\mathrm{H}(2)$

$\mathrm{S}(2)-\operatorname{Ir}(1)-\mathrm{H}(2)$

$\mathrm{H}(1)-\operatorname{Ir}(1)-\mathrm{H}(2)$

$\mathrm{P}(3)-\mathrm{S}(2)-\operatorname{Ir}(1)$

$\mathrm{C}(1)-\mathrm{P}(1)-\mathrm{C}(6)$

$\mathrm{C}(1)-\mathrm{P}(1)-\operatorname{Ir}(1)$

$C(6)-P(1)-\operatorname{Ir}(1)$

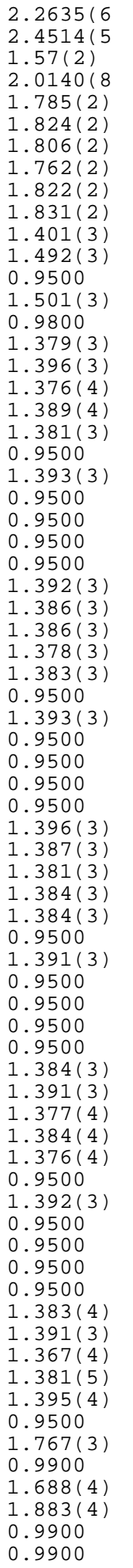

$\operatorname{Ir}(1)-P(4)$

$\operatorname{Ir}(1)-S(1)$

$\operatorname{Ir}(1)-\mathrm{H}(2)$

$\mathrm{S}(2)-\mathrm{P}(3)$

$P(1)-C(5)$

$P(2)-C(1)$

$P(2)-C(7)$

$P(3)-C(31)$

$P(4)-C(55)$

$P(4)-C(43)$

$\mathrm{C}(2)-\mathrm{C}(3)$

$C(3)-C(4)$

$C(4)-C(5)$

$\mathrm{C}(6)-\mathrm{H}(6 \mathrm{~A})$

$\mathrm{C}(6)-\mathrm{H}(6 \mathrm{C})$

$\mathrm{C}(7)-\mathrm{C}(12)$

$\mathrm{C}(8)-\mathrm{H}(8)$

$\mathrm{C}(9)-\mathrm{H}(9)$

$\mathrm{C}(10)-\mathrm{H}(10)$

$\mathrm{C}(11)-\mathrm{H}(11)$

C (13) $-\mathrm{C}(18)$

$\mathrm{C}(14)-\mathrm{C}(15)$

$\mathrm{C}(15)-\mathrm{C}(16)$

$C(16)-C(17)$

$C(17)-C(18)$

$\mathrm{C}(18)-\mathrm{H}(18)$

C (19) $-\mathrm{C}(24)$

$\mathrm{C}(20)-\mathrm{H}(20)$

$\mathrm{C}(21)-\mathrm{H}(21)$

$\mathrm{C}(22)-\mathrm{H}(22)$

$\mathrm{C}(23)-\mathrm{H}(23)$

C (25) $-\mathrm{C}(26)$

C (26) $-\mathrm{C}(27)$

$C(27)-C(28)$

$C(28)-C(29)$

C (29)-C (30)

$\mathrm{C}(30)-\mathrm{H}(30)$

C (31) $-\mathrm{C}(32)$

$\mathrm{C}(32)-\mathrm{H}(32)$

$\mathrm{C}(33)-\mathrm{H}(33)$

$\mathrm{C}(34)-\mathrm{H}(34)$

$\mathrm{C}(35)-\mathrm{H}(35)$

$C(37)-C(42)$

C (38) $-\mathrm{C}(39)$

$C(39)-C(40)$

C (40) $-C(41)$

C (41) $-\mathrm{C}(42)$

$\mathrm{C}(42)-\mathrm{H}(42)$

C (43)-C (48)

$\mathrm{C}(44)-\mathrm{H}(44)$

$\mathrm{C}(45)-\mathrm{H}(45)$

$\mathrm{C}(46)-\mathrm{H}(46)$

$\mathrm{C}(47)-\mathrm{H}(47)$

C (49) $-\mathrm{C}(50)$

$C(50)-C(51)$

C (51) $-C(52)$

$C(52)-C(53)$

C (53) $-\mathrm{C}(54)$

$\mathrm{C}(54)-\mathrm{H}(54)$

$C(55)-C(56)$

$\mathrm{C}(56)-\mathrm{H}(56)$

$\mathrm{C}(57)-\mathrm{H}(57)$

$\mathrm{C}(58)-\mathrm{H}(58)$

C (59) $-\mathrm{H}(59)$

$\mathrm{C}(61)-\mathrm{Cl}$ (1)

$\mathrm{C}(61)-\mathrm{H}(61 \mathrm{~A})$

C (62) $-\mathrm{Cl}(3 A)$

$C(62)-C l(4 A)$

$\mathrm{C}(62)-\mathrm{H}(62 \mathrm{~A})$

$\mathrm{C}(62)-\mathrm{H}(62 \mathrm{C})$

$2.2920(6)$

2. $4717(6)$

1.54(2)

$2.0127(7)$

$1.799(2)$

$1.771(2)$

$1.820(2)$

$1.806(2)$

$1.830(2)$

$1.837(2)$

$1.417(3)$

1.406 (3)

1. 402 (3)

0.9800

0.9800

1.398 (3)

0.9500

0.9500

0.9500

0.9500

1.392 (3)

$1.386(3)$

$1.387(3)$

$1.383(3)$

$1.387(3)$

0.9500

1. 400 (3)

0.9500

0.9500

0.9500

0.9500

1.391 (3)

$1.392(3)$

$1.376(3)$

$1.377(3)$

1. 389 (3)

0.9500

1.399(3)

0.9500

0.9500

0.9500

0.9500

$1.389(3)$

1. 381 (3)

1.381 (4)

$1.387(4)$

$1.395(3)$

0.9500

1. 400 (3)

0.9500

0.9500

0.9500

0.9500

1. 387 (3)

$1.386(4)$

$1.352(5)$

$1.382(5)$

1. 375 (4)

0.9500

1.397(4)

0.9500

0.9500

0.9500

0.9500

1.758 (3)

0.9900

1.506 (5)

1. $779(6)$

0.9900

0.9900

$\begin{array}{llr}176.70(2) & \mathrm{P}(1)-\operatorname{Ir}(1)-\mathrm{S}(2) & 89.27(2) \\ 92.23(2) & \mathrm{P}(1)-\operatorname{Ir}(1)-\mathrm{S}(1) & 89.20(2) \\ 93.73(2) & \mathrm{S}(2)-\operatorname{Ir}(1)-\mathrm{S}(1) & 90.14(2) \\ 85.5(8) & \mathrm{P}(4)-\operatorname{Ir}(1)-\mathrm{H}(1) & 91.5(8) \\ 91.3(8) & \mathrm{S}(1)-\operatorname{Ir}(1)-\mathrm{H}(1) & 174.5(8) \\ 89.4(8) & \mathrm{P}(4)-\operatorname{Ir}(1)-\mathrm{H}(2) & 89.0(8) \\ 177.0(8) & \mathrm{S}(1)-\operatorname{Ir}(1)-\mathrm{H}(2) & 92.6(8) \\ 86(1) & \mathrm{P}(2)-\mathrm{S}(1)-\operatorname{Ir}(1) & 98.28(3) \\ 98.43(3) & \mathrm{C}(1)-\mathrm{P}(1)-\mathrm{C}(5) & 101.8(1) \\ 104.7(1) & \mathrm{C}(5)-\mathrm{P}(1)-\mathrm{C}(6) & 104.3(1) \\ 114.03(7) & \mathrm{C}(5)-\mathrm{P}(1)-\operatorname{Ir}(1) & 113.47(7) \\ 116.87(8) & \mathrm{C}(1)-\mathrm{P}(2)-\mathrm{C}(13) & 112.2(1)\end{array}$


$110.3(1)$

$110.15(8)$

$108.53(8)$

$109.9(1)$

$109.60(7)$

$108.71(7)$

$103.4(1)$

$116.01(8)$

$114.46(7)$

117.8 (1)

$121.5(2)$

$116.4(2)$

117.1

122.1 (2)

$116.9(2)$

$117.2(2)$

109.5

109.5

109.5

$119.3(2)$

$119.6(2)$

119.6

$119.6(2)$

120.2

120.1

$120.6(2)$

119.7

120.1

$119.6(2)$

$116.6(2)$

120.0

120.2 (2)

119.9

120.0

120.2 (2)

119.9

120.0

$117.6(2)$

121.1 (2)

119.4

$120.2(2)$

119.9

120.2

$120.3(2)$

119.9

119.4

$117.8(2)$

$121.5(2)$

119.3

$119.6(2)$

120.2

120.0

$120.4(2)$

119.8

119.6

$119.3(2)$

$123.0(2)$

120.1

$120.6(2)$

119.7

120.1

$120.5(2)$

119.7

120.0

$119.0(2)$

121.1 (2)

119.5

$120.0(2)$

120.0

120.1

120.2 (2)

119.9

120.0

$118.2(2)$

$118.8(2)$

119.7

$120.5(3)$

119.7

120.2

$120.0(3)$

120.0

119.4

$118.6(2)$

118.7 (2)

120.3

$121.3(3)$

119.4
(13) $-\mathrm{P}(2)-\mathrm{C}(7)$

(13) $-P(2)-S(1)$

$\mathrm{C}(5)-\mathrm{P}(3)-\mathrm{C}(31)$

C (31) $-\mathrm{P}(3)-\mathrm{C}(37)$

$\mathrm{C}(31)-\mathrm{P}(3)-\mathrm{S}(2)$

$C(55)-P(4)-C(49)$

$C(49)-P(4)-C(43)$

C (49)-P (4)-Ir (1)

$C(2)-C(1)-P(2)$

$P(2)-C(1)-P(1)$

$C(1)-C(2)-C(19)$

$C(4)-C(3)-C(2)$

$\mathrm{C}(2)-\mathrm{C}(3)-\mathrm{H}(3)$

$C(5)-C(4)-C(25)$

( $(4)-C(5)-P(3)$

$P(3)-C(5)-P(1)$

$\mathrm{P}(1)-\mathrm{C}(6)-\mathrm{H}(6 \mathrm{~B})$

$P(1)-C(6)-H(6 C)$

$\mathrm{H}(6 \mathrm{~B})-\mathrm{C}(6)-\mathrm{H}(6 \mathrm{C})$

$\mathrm{C}(8)-\mathrm{C}(7)-\mathrm{P}(2)$

$C(7)-C(8)-C(9)$

$\mathrm{C}(9)-\mathrm{C}(8)-\mathrm{H}(8)$

$\mathrm{C}(10)-\mathrm{C}(9)-\mathrm{H}(9)$

$C(9)-C(10)-C(11)$

$\mathrm{C}(11)-\mathrm{C}(10)-\mathrm{H}(10)$

$\mathrm{C}(12)-\mathrm{C}(11)-\mathrm{H}(11)$

$C(11)-C(12)-C(7)$

$\mathrm{C}(7)-\mathrm{C}(12)-\mathrm{H}(12)$

$\mathrm{C}(18)-\mathrm{C}(13)-\mathrm{P}(2)$

$\mathrm{C}(15)-\mathrm{C}(14)-\mathrm{C}(13)$

$\mathrm{C}(13)-\mathrm{C}(14)-\mathrm{H}(14)$

$\mathrm{C}(14)-\mathrm{C}(15)-\mathrm{H}(15)$

$C(17)-C(16)-C(15)$

$C(15)-\mathrm{C}(16)-\mathrm{H}(16)$

$\mathrm{C}(16)-\mathrm{C}(17)-\mathrm{H}(17)$

$\mathrm{C}(17)-\mathrm{C}(18)-\mathrm{C}(13)$

$\mathrm{C}(13)-\mathrm{C}(18)-\mathrm{H}(18)$

$C(20)-C(19)-C(2)$

$\mathrm{C}(21)-\mathrm{C}(20)-\mathrm{C}(19)$

$\mathrm{C}(19)-\mathrm{C}(20)-\mathrm{H}(20)$

$\mathrm{C}(22)-\mathrm{C}(21)-\mathrm{H}(21)$

$C(23)-C(22)-C(21)$

(21) $-\mathrm{C}(22)-\mathrm{H}(22)$

$\mathrm{C}(22)-\mathrm{C}(23)-\mathrm{H}(23)$

C (23) $-\mathrm{C}(24)-\mathrm{C}(19)$

$\mathrm{C}(19)-\mathrm{C}(24)-\mathrm{H}(24)$

$C(26)-C(25)-C(4)$

$\mathrm{C}(25)-\mathrm{C}(26)-\mathrm{C}(27)$

$\mathrm{C}(27)-\mathrm{C}(26)-\mathrm{H}(26)$

$\mathrm{C}(28)-\mathrm{C}(27)-\mathrm{H}(27)$

C (27) $-\mathrm{C}(28)-\mathrm{C}(29)$

$\mathrm{C}(29)-\mathrm{C}(28)-\mathrm{H}(28)$

$\mathrm{C}(28)-\mathrm{C}(29)-\mathrm{H}(29)$

C (29) $-\mathrm{C}(30)-\mathrm{C}(25)$

$\mathrm{C}(25)-\mathrm{C}(30)-\mathrm{H}(30)$

$\mathrm{C}(36)-\mathrm{C}(31)-\mathrm{P}(3)$

$C(33)-C(32)-C(31)$

$\mathrm{C}(31)-\mathrm{C}(32)-\mathrm{H}(32)$

$\mathrm{C}(34)-\mathrm{C}(33)-\mathrm{H}(33)$

C (33) $-\mathrm{C}(34)-\mathrm{C}(35)$

$\mathrm{C}(35)-\mathrm{C}(34)-\mathrm{H}(34)$

C (34) $-\mathrm{C}(35)-\mathrm{H}(35)$

C (35) $-\mathrm{C}(36)-\mathrm{C}(31)$

$\mathrm{C}(31)-\mathrm{C}(36)-\mathrm{H}(36)$

$C(42)-C(37)-P(3)$

$C(39)-C(38)-C(37)$

$\mathrm{C}(37)-\mathrm{C}(38)-\mathrm{H}(38)$

$\mathrm{C}(40)-\mathrm{C}(39)-\mathrm{H}(39)$

C (39) $-\mathrm{C}(40)-\mathrm{C}(41)$

C $(41)-\mathrm{C}(40)-\mathrm{H}(40)$

$\mathrm{C}(40)-\mathrm{C}(41)-\mathrm{H}(41)$

C (37) $-\mathrm{C}(42)-\mathrm{C}(41)$

$\mathrm{C}(41)-\mathrm{C}(42)-\mathrm{H}(42)$

C (44)-C (43)-P (4)

$C(43)-C(44)-C(45)$

$\mathrm{C}(45)-\mathrm{C}(44)-\mathrm{H}(44)$

$\mathrm{C}(46)-\mathrm{C}(45)-\mathrm{H}(45)$

C (45) $-\mathrm{C}(46)-\mathrm{C}(47)$

$\mathrm{C}(47)-\mathrm{C}(46)-\mathrm{H}(46)$

$\mathrm{C}(48)-\mathrm{C}(47)-\mathrm{H}(47)$

$C(47)-C(48)-C(43)$

$\mathrm{C}(43)-\mathrm{C}(48)-\mathrm{H}(48)$

C $(50)-C(49)-P(4)$

$C(51)-C(50)-C(49)$

$\mathrm{C}(49)-\mathrm{C}(50)-\mathrm{H}(50)$

$\mathrm{C}(52)-\mathrm{C}(51)-\mathrm{H}(51)$

$\mathrm{C}(51)-\mathrm{C}(52)-\mathrm{C}(53)$
$109.1(1)$

$106.45(7)$

$114.4(1)$

$109.2(1)$

$104.80(7)$

$103.1(1)$

$102.1(1)$

$115.86(8)$

$129.5(2)$

$111.7(1)$

$122.2(2)$

$125.9(2)$

117.1

$121.0(2)$

$130.9(2)$

$111.3(1)$

109.5

109.5

109.5

$120.9(2)$

$120.8(2)$

119.6

120.2

$119.9(2)$

120.1

119.7
$119.8(2)$

120.1

$123.7(2)$

$120.0(2)$

120.0

119.9

$119.9(2)$

120.0

119.9

120.1 (2)

120.0

$121.3(2)$

$121.2(2)$

119.4

119.9

119.5 (2)

120.2

119.9

$121.2(2)$

119.4

120.7 (2)

$121.5(2)$

119.3

120.2

$119.9(2)$

120.0

119.8

$120.8(2)$

119.6

$117.2(2)$

119.8 (2)

120.1

119.7

119.7 (2)

120.1

119.7

120.0 (2)

120.0

119.7 (2)

$120.9(2)$

119.5

120.0

119.8 (2)

120.1

119.9

$120.0(2)$

120.0

$122.9(2)$

$120.6(2)$

119.7

119.7

$119.6(3)$

120.2

120.0

121.1 (2)

119.4

$122.7(2)$

119.5 (3)

120.3

119.4

120.1 (3) 
C (51) $-\mathrm{C}(52)-\mathrm{H}(52)$

$C(54)-C(53)-C(52)$

$\mathrm{C}(52)-\mathrm{C}(53)-\mathrm{H}(53)$

$\mathrm{C}(53)-\mathrm{C}(54)-\mathrm{H}(54)$

$C(60)-C(55)-C(56)$

$\mathrm{C}(56)-\mathrm{C}(55)-\mathrm{P}(4)$

$\mathrm{C}(57)-\mathrm{C}(56)-\mathrm{H}(56)$

C $(58)-C(57)-C(56)$

$\mathrm{C}(56)-\mathrm{C}(57)-\mathrm{H}(57)$

$\mathrm{C}(57)-\mathrm{C}(58)-\mathrm{H}(58)$

$C(58)-C(59)-C(60)$

$\mathrm{C}(60)-\mathrm{C}(59)-\mathrm{H}(59)$

$\mathrm{C}(55)-\mathrm{C}(60)-\mathrm{H}(60)$

$\mathrm{Cl}(1)-\mathrm{C}(61)-\mathrm{Cl}(2)$

$\mathrm{Cl}(2)-\mathrm{C}(61)-\mathrm{H}(61 \mathrm{~A})$

$\mathrm{Cl}(2)-\mathrm{C}(61)-\mathrm{H}(61 \mathrm{~B})$

$\mathrm{Cl}(3 \mathrm{~A})-\mathrm{C}(62)-\mathrm{Cl}(4)$

$\mathrm{Cl}(4)-\mathrm{C}(62)-\mathrm{Cl}(4 \mathrm{~A}$

$\mathrm{Cl}(4)-\mathrm{C}(62)-\mathrm{Cl}(3)$

$\mathrm{Cl}(3 \mathrm{~A})-\mathrm{C}(62)-\mathrm{H}(62 \mathrm{~A})$

$\mathrm{Cl}(4 \mathrm{~A})-\mathrm{C}(62)-\mathrm{H}(62 \mathrm{~A})$

$\mathrm{Cl}(3 \mathrm{~A})-\mathrm{C}(62)-\mathrm{H}(62 \mathrm{~B})$

$\mathrm{Cl}(4 \mathrm{~A})-\mathrm{C}(62)-\mathrm{H}(62 \mathrm{~B})$

$\mathrm{H}(62 \mathrm{~A})-\mathrm{C}(62)-\mathrm{H}(62 \mathrm{~B})$

$\mathrm{Cl}(4)-\mathrm{C}(62)-\mathrm{H}(62 \mathrm{C})$

$\mathrm{Cl}(3)-\mathrm{C}(62)-\mathrm{H}(62 \mathrm{C})$

$\mathrm{H}(62 \mathrm{~B})-\mathrm{C}(62)-\mathrm{H}(62 \mathrm{C})$

$\mathrm{Cl}(4)-\mathrm{C}(62)-\mathrm{H}(62 \mathrm{D})$

$\mathrm{Cl}(3)-\mathrm{C}(62)-\mathrm{H}(62 \mathrm{D})$

$\mathrm{H}(62 \mathrm{~B})-\mathrm{C}(62)-\mathrm{H}(62 \mathrm{D})$
119.9

$119.4(3)$

120.3

119.5

$118.6(2)$

$117.8(2)$

119.7

120.1 (3)

120.0

119.9

120.1 (3)

120.0

119.8

$111.7(2)$

109.3

109.3

$133.6(3)$

$20.8(2)$

$109.9(2)$

99.6

101.7

93.5

130.2

108.2

87.1

115.9

22.9

110.0

121.6

94.7
C (53) $-\mathrm{C}(52)-\mathrm{H}(52)$

C (54) $-\mathrm{C}(53)-\mathrm{H}(53)$

C (53) $-\mathrm{C}(54)-\mathrm{C}(49)$

$\mathrm{C}(49)-\mathrm{C}(54)-\mathrm{H}(54)$

$C(60)-C(55)-P(4)$

$C(57)-C(56)-C(55)$

$\mathrm{C}(55)-\mathrm{C}(56)-\mathrm{H}(56)$

$\mathrm{C}(58)-\mathrm{C}(57)-\mathrm{H}(57)$

C $(57)-C(58)-C(59)$

$\mathrm{C}(59)-\mathrm{C}(58)-\mathrm{H}(58)$

$\mathrm{C}(58)-\mathrm{C}(59)-\mathrm{H}(59)$

$C(55)-C(60)-C(59)$

$\mathrm{C}(59)-\mathrm{C}(60)-\mathrm{H}(60)$

$\mathrm{Cl}(1)-\mathrm{C}(61)-\mathrm{H}(61 \mathrm{~A})$

$\mathrm{Cl}(1)-\mathrm{C}(61)-\mathrm{H}(61 \mathrm{~B})$

$H(61 A)-C(61)-H(61 B)$

$\mathrm{Cl}(3 \mathrm{~A})-\mathrm{C}(62)-\mathrm{Cl}(4 \mathrm{~A})$

$\mathrm{Cl}(3 \mathrm{~A})-\mathrm{C}(62)-\mathrm{Cl}(3)$

$\mathrm{Cl}(4 \mathrm{~A})-\mathrm{C}(62)-\mathrm{Cl}(3)$

$\mathrm{Cl}(4)-\mathrm{C}(62)-\mathrm{H}(62 \mathrm{~A})$

$\mathrm{Cl}(3)-\mathrm{C}(62)-\mathrm{H}(62 \mathrm{~A})$

$\mathrm{Cl}(4)-\mathrm{C}(62)-\mathrm{H}(62 \mathrm{~B})$

$\mathrm{Cl}(3)-\mathrm{C}(62)-\mathrm{H}(62 \mathrm{~B})$

$\mathrm{Cl}(3 \mathrm{~A})-\mathrm{C}(62)-\mathrm{H}(62 \mathrm{C})$

$\mathrm{Cl}(4 \mathrm{~A})-\mathrm{C}(62)-\mathrm{H}(62 \mathrm{C})$

$\mathrm{H}(62 \mathrm{~A})-\mathrm{C}(62)-\mathrm{H}(62 \mathrm{C})$

$\mathrm{Cl}(3 \mathrm{~A})-\mathrm{C}(62)-\mathrm{H}(62 \mathrm{D})$

$\mathrm{Cl}(4 \mathrm{~A})-\mathrm{C}(62)-\mathrm{H}(62 \mathrm{D})$

$\mathrm{H}(62 \mathrm{~A})-\mathrm{C}(62)-\mathrm{H}(62 \mathrm{D})$

$\mathrm{H}(62 \mathrm{C})-\mathrm{C}(62)-\mathrm{H}(62 \mathrm{D})$
119.9

120.3

$121.1(3)$

119.5

$123.6(2)$

$120.6(3)$

119.7

120.0

$120.2(3)$

119.9

120.0

$120.4(3)$

119.8

109.3

109.3

108.0

$119.9(3)$

$24.1(2)$

$96.0(2)$

109.7

109.7

109.7

109.7

107.3

107.3

121.7

107.3

107.3

14.9

106.9 
Table 4. Anisotropic displacement parameters ( $\left.A^{\wedge} 2 \times 10^{\wedge} 3\right)$ for

\begin{tabular}{|c|c|c|c|c|c|c|}
\hline atom & U11 & U22 & U33 & U23 & U13 & $\mathrm{U} 12$ \\
\hline $\operatorname{Ir}(1)$ & $16(1)$ & $14(1)$ & $17(1)$ & $1(1)$ & $-3(1)$ & $-4(1)$ \\
\hline$S(1)$ & $17(1)$ & $21(1)$ & $23(1)$ & $-4(1)$ & $-3(1)$ & $-6(1)$ \\
\hline$S(2)$ & $21(1)$ & $20(1)$ & $18(1)$ & $0(1)$ & $-4(1)$ & $-8(1)$ \\
\hline P (1) & $14(1)$ & $15(1)$ & $16(1)$ & $1(1)$ & $-4(1)$ & $-4(1)$ \\
\hline P (2) & $15(1)$ & $17(1)$ & $16(1)$ & $1(1)$ & $-3(1)$ & $-5(1)$ \\
\hline$P(3)$ & $15(1)$ & 17 (1) & $16(1)$ & $1(1)$ & $-3(1)$ & $-5(1)$ \\
\hline$P(4)$ & $22(1)$ & 15 (1) & $24(1)$ & $-1(1)$ & $-1(1)$ & $-5(1)$ \\
\hline$C(1)$ & $15(1)$ & $18(1)$ & $16(1)$ & $0(1)$ & $-2(1)$ & $-5(1)$ \\
\hline$C(2)$ & $18(1)$ & $19(1)$ & $13(1)$ & $0(1)$ & $-5(1)$ & $-3(1)$ \\
\hline$C(3)$ & $21(1)$ & $16(1)$ & $20(1)$ & $-2(1)$ & $-5(1)$ & $-6(1)$ \\
\hline$C(4)$ & $18(1)$ & $19(1)$ & $16(1)$ & $1(1)$ & $-6(1)$ & $-7(1)$ \\
\hline$C(5)$ & $15(1)$ & $17(1)$ & $17(1)$ & $2(1)$ & $-5(1)$ & $-6(1)$ \\
\hline$C(6)$ & $23(1)$ & $26(1)$ & 21 (1) & $2(1)$ & $-9(1)$ & $-8(1)$ \\
\hline$C(7)$ & $18(1)$ & 21 (1) & $19(1)$ & $5(1)$ & $-1(1)$ & $-4(1)$ \\
\hline$C(8)$ & $26(1)$ & $25(1)$ & $19(1)$ & $1(1)$ & $-4(1)$ & $-3(1)$ \\
\hline$C(9)$ & $40(2)$ & $39(2)$ & $23(1)$ & $2(1)$ & $-10(1)$ & $-1(1)$ \\
\hline C (10) & $41(2)$ & $41(2)$ & $30(1)$ & $17(1)$ & $-2(1)$ & $-4(1)$ \\
\hline C (11) & $38(2)$ & $35(2)$ & $42(2)$ & $18(1)$ & $-3(1)$ & $-14(1)$ \\
\hline C (12) & $30(1)$ & $28(1)$ & $35(1)$ & $7(1)$ & $-7(1)$ & $-13(1)$ \\
\hline$C(13)$ & $17(1)$ & $18(1)$ & $20(1)$ & $-3(1)$ & $-4(1)$ & $-6(1)$ \\
\hline$C(14)$ & $21(1)$ & $23(1)$ & $23(1)$ & $2(1)$ & $-4(1)$ & $-6(1)$ \\
\hline$C(15)$ & $28(1)$ & $24(1)$ & $29(1)$ & $3(1)$ & $-12(1)$ & $-4(1)$ \\
\hline$C(16)$ & $20(1)$ & $28(1)$ & $37(1)$ & $-6(1)$ & $-10(1)$ & $2(1)$ \\
\hline$C(17)$ & $19(1)$ & $38(1)$ & 31 (1) & $-4(1)$ & $-1(1)$ & $-3(1)$ \\
\hline$C(18)$ & $20(1)$ & $32(1)$ & $21(1)$ & $1(1)$ & $-2(1)$ & $-5(1)$ \\
\hline C (19) & $18(1)$ & $16(1)$ & $19(1)$ & $-3(1)$ & $-4(1)$ & $-6(1)$ \\
\hline C (20) & $24(1)$ & $23(1)$ & $23(1)$ & $1(1)$ & $-4(1)$ & $-4(1)$ \\
\hline C (21) & $28(1)$ & $26(1)$ & $30(1)$ & $-2(1)$ & $-8(1)$ & $3(1)$ \\
\hline C (22) & $19(1)$ & $32(1)$ & $27(1)$ & $-11(1)$ & $-3(1)$ & $1(1)$ \\
\hline C (23) & $30(1)$ & $33(1)$ & 21 (1) & $-5(1)$ & $0(1)$ & $-7(1)$ \\
\hline C (24) & $26(1)$ & 21 (1) & 21 (1) & $-1(1)$ & $-2(1)$ & $-4(1)$ \\
\hline$C(25)$ & $20(1)$ & $18(1)$ & $17(1)$ & $-1(1)$ & $-5(1)$ & $-8(1)$ \\
\hline$C(26)$ & $22(1)$ & 17 (1) & $28(1)$ & $1(1)$ & $-6(1)$ & $-7(1)$ \\
\hline$C(27)$ & $21(1)$ & $25(1)$ & $34(1)$ & $-1(1)$ & $1(1)$ & $-9(1)$ \\
\hline$C(28)$ & 31 (1) & $23(1)$ & $28(1)$ & $5(1)$ & $-4(1)$ & $-13(1)$ \\
\hline C (29) & $30(1)$ & 19 (1) & $34(1)$ & $8(1)$ & $-9(1)$ & $-7(1)$ \\
\hline$C(30)$ & $22(1)$ & 21 (1) & $29(1)$ & $2(1)$ & $-5(1)$ & $-5(1)$ \\
\hline$C(31)$ & $18(1)$ & $19(1)$ & $16(1)$ & $2(1)$ & $-4(1)$ & $-8(1)$ \\
\hline C (32) & $20(1)$ & $28(1)$ & $23(1)$ & $4(1)$ & $-4(1)$ & $-8(1)$ \\
\hline C (33) & $26(1)$ & $38(1)$ & $23(1)$ & $6(1)$ & $-1(1)$ & $-14(1)$ \\
\hline C (34) & $36(1)$ & $32(1)$ & $23(1)$ & $9(1)$ & $-11(1)$ & $-14(1)$ \\
\hline C (35) & $27(1)$ & $32(1)$ & $30(1)$ & $9(1)$ & $-13(1)$ & $-7(1)$ \\
\hline$C(36)$ & $19(1)$ & $26(1)$ & $26(1)$ & $3(1)$ & $-4(1)$ & $-4(1)$ \\
\hline$C(37)$ & $16(1)$ & $19(1)$ & $21(1)$ & $2(1)$ & $-4(1)$ & $-5(1)$ \\
\hline C (38) & 21 (1) & $20(1)$ & $23(1)$ & $2(1)$ & $-5(1)$ & $-7(1)$ \\
\hline$C(39)$ & $22(1)$ & $32(1)$ & $31(1)$ & $6(1)$ & $-11(1)$ & $-11(1)$ \\
\hline$C(40)$ & $18(1)$ & 31 (1) & $41(2)$ & $5(1)$ & $-9(1)$ & $-2(1)$ \\
\hline$C(41)$ & $23(1)$ & 32 (1) & $40(2)$ & $-6(1)$ & $-2(1)$ & $2(1)$ \\
\hline$C(42)$ & $23(1)$ & $30(1)$ & $25(1)$ & $-4(1)$ & $-6(1)$ & $-2(1)$ \\
\hline$C(43)$ & $27(1)$ & 19 (1) & $26(1)$ & $-2(1)$ & $-3(1)$ & $-11(1)$ \\
\hline$C(44)$ & $33(1)$ & $23(1)$ & $27(1)$ & $-2(1)$ & $-3(1)$ & $-5(1)$ \\
\hline$C(45)$ & $44(2)$ & $33(1)$ & $30(1)$ & $-8(1)$ & $-3(1)$ & $-6(1)$ \\
\hline$C(46)$ & $49(2)$ & $40(2)$ & $32(2)$ & $-5(1)$ & $-14(1)$ & $-15(1)$ \\
\hline$C(47)$ & $39(2)$ & $39(2)$ & $42(2)$ & $-7(1)$ & $-20(1)$ & $-9(1)$ \\
\hline C (48) & $25(1)$ & $33(1)$ & $37(2)$ & $-9(1)$ & $-7(1)$ & $-5(1)$ \\
\hline$C(49)$ & $34(1)$ & $19(1)$ & $23(1)$ & $-4(1)$ & $-7(1)$ & $4(1)$ \\
\hline$C(50)$ & $60(2)$ & $25(1)$ & $34(2)$ & $2(1)$ & $-9(1)$ & $4(1)$ \\
\hline$C(51)$ & $81(3)$ & $30(2)$ & $46(2)$ & $3(1)$ & $-20(2)$ & $18(2)$ \\
\hline$C(52)$ & $58(2)$ & $64(2)$ & $37(2)$ & $-18(2)$ & $-23(2)$ & $34(2)$ \\
\hline$C(53)$ & $33(2)$ & $61(2)$ & $49(2)$ & $-21(2)$ & $-11(1)$ & $14(1)$ \\
\hline$C(54)$ & $28(1)$ & $36(2)$ & $43(2)$ & $-8(1)$ & $-4(1)$ & $3(1)$ \\
\hline$C(55)$ & $34(1)$ & $20(1)$ & $35(1)$ & $-3(1)$ & $5(1)$ & $-11(1)$ \\
\hline$C(56)$ & $59(2)$ & $40(2)$ & $37(2)$ & $3(1)$ & $0(1)$ & $-28(2)$ \\
\hline$C(57)$ & $72(2)$ & $49(2)$ & $36(2)$ & $2(1)$ & $8(2)$ & $-31(2)$ \\
\hline C (58) & $69(2)$ & $54(2)$ & $58(2)$ & $-7(2)$ & $19(2)$ & $-41(2)$ \\
\hline C (59) & $84(3)$ & $67(2)$ & $65(2)$ & $-17(2)$ & $15(2)$ & $-59(2)$ \\
\hline$C(60)$ & $64(2)$ & $52(2)$ & $43(2)$ & $-12(1)$ & $10(2)$ & $-40(2)$ \\
\hline$C(61)$ & $46(2)$ & $57(2)$ & $32(2)$ & $-14(1)$ & $2(1)$ & $-21(2)$ \\
\hline $\mathrm{Cl}(1)$ & $43(1)$ & $83(1)$ & $43(1)$ & $-14(1)$ & $-6(1)$ & $-24(1)$ \\
\hline $\mathrm{Cl}(2)$ & $55(1)$ & $68(1)$ & $34(1)$ & $-10(1)$ & $-5(1)$ & $-15(1)$ \\
\hline$C(62)$ & $94(3)$ & $49(2)$ & $61(2)$ & $7(2)$ & $-46(2)$ & $-17(2)$ \\
\hline $\mathrm{Cl}(3)$ & $73(1)$ & $94(2)$ & $45(1)$ & $-7(1)$ & $-1(1)$ & $-38(1)$ \\
\hline $\operatorname{Cl}(4)$ & $79(2)$ & $61(1)$ & $73(2)$ & $14(1)$ & $-1(1)$ & $-26(1)$ \\
\hline $\mathrm{Cl}(3 \mathrm{~A})$ & $73(1)$ & $94(2)$ & $45(1)$ & $-7(1)$ & $-1(1)$ & $-38(1)$ \\
\hline $\operatorname{Cl}(4 A)$ & $79(2)$ & $61(1)$ & $73(2)$ & $14(1)$ & $-1(1)$ & $-26(1)$ \\
\hline
\end{tabular}

The anisotropic displacement factor exponent takes the form

$2 \mathrm{pi}^{\wedge} 2\left[\mathrm{~h}^{\wedge} 2 \mathrm{a} \star \wedge 2 \mathrm{U}(11)+\ldots+2 \mathrm{hka} \mathrm{b}^{\star} \mathrm{U}(12)\right]$ 
Table 5. Hydrogen Coordinates (A $\times 10^{\wedge} 4$ ) and equivalent isotropic displacement parameters $\left(A^{\wedge} 2 \times 10^{\wedge} 3\right)$ for 4

\begin{tabular}{|c|c|c|c|c|}
\hline atom & $x$ & y & z & $\mathrm{U}(\mathrm{eq})$ \\
\hline $\mathrm{H}(1)$ & $2480(20)$ & $2330(20)$ & $7640(10)$ & 19 \\
\hline $\mathrm{H}(2)$ & $860(20)$ & $2220(20)$ & $8440(10)$ & 19 \\
\hline $\mathrm{H}(3)$ & -41 & 6876 & 8029 & 22 \\
\hline$H(6 A)$ & 2672 & 3793 & 8534 & 34 \\
\hline$H(6 B)$ & 1443 & 3784 & 9152 & 34 \\
\hline $\mathrm{H}(6 \mathrm{C})$ & 1676 & 4786 & 8807 & 34 \\
\hline $\mathrm{H}(8)$ & -710.9999 & 4112 & 9742 & 29 \\
\hline $\mathrm{H}(9)$ & -619 & 3195 & 10834 & 42 \\
\hline $\mathrm{H}(10)$ & -1652 & 1982 & 11097 & 49 \\
\hline $\mathrm{H}(11)$ & -2777 & 1685 & 10274 & 48 \\
\hline $\mathrm{H}(12)$ & -2820 & 2563 & 9171 & 36 \\
\hline $\mathrm{H}(14)$ & -2709 & 5297 & 7383 & 27 \\
\hline $\mathrm{H}(15)$ & -4692 & 6212 & 7235 & 32 \\
\hline H (16) & -6454 & 6231 & 8169 & 35 \\
\hline H (17) & -6230.0005 & 5360 & 9262 & 37 \\
\hline $\mathrm{H}(18)$ & -4248 & 4465 & 9425 & 30 \\
\hline $\mathrm{H}(20)$ & -2290 & 7458 & 7966 & 29 \\
\hline $\mathrm{H}(21)$ & -4059 & 8488 & 8655 & 35 \\
\hline $\mathrm{H}(22)$ & -4914 & 8040 & 9849 & 33 \\
\hline $\mathrm{H}(23)$ & -3917.9998 & 6594 & 10369 & 34 \\
\hline $\mathrm{H}(24)$ & -2134 & 5572 & 9690 & 28 \\
\hline $\mathrm{H}(26)$ & 3492 & 5618 & 6745 & 27 \\
\hline $\mathrm{H}(27)$ & 4493 & 6653 & 5975 & 33 \\
\hline $\mathrm{H}(28)$ & 3323 & 8162 & 5649 & 32 \\
\hline H (29) & 1161 & 8624 & 6077 & 34 \\
\hline $\mathrm{H}(30)$ & 150 & 7580 & 6825 & 29 \\
\hline $\mathrm{H}(32)$ & 4256 & 4457 & 5201 & 28 \\
\hline H (33) & 4167 & 5230 & 4057 & 35 \\
\hline $\mathrm{H}(34)$ & 2242 & 6035 & 3770 & 35 \\
\hline $\mathrm{H}(35)$ & 392 & 6126 & 4645 & 35 \\
\hline $\mathrm{H}(36)$ & 455 & 5378 & 5797 & 29 \\
\hline $\mathrm{H}(38)$ & 3919 & 4266 & 7442 & 25 \\
\hline $\mathrm{H}(39)$ & 5881 & 3384 & 7628 & 33 \\
\hline $\mathrm{H}(40)$ & 7070 & 2120 & 6855 & 37 \\
\hline H ( 41) & 6307 & 1766 & 5875 & 41 \\
\hline $\mathrm{H}(42)$ & 4305 & 2627 & 5705 & 32 \\
\hline $\mathrm{H}(44)$ & 2812 & 10 & 5958 & 35 \\
\hline $\mathrm{H}(45)$ & 2585 & -7.0000 & 4747 & 44 \\
\hline $\mathrm{H}(46)$ & 758 & 901 & 4394 & 46 \\
\hline $\mathrm{H}(47)$ & -851 & 1849 & 5256 & 46 \\
\hline $\mathrm{H}(48)$ & -611 & 1902 & 6452 & 38 \\
\hline $\mathrm{H}(50)$ & 2159 & -1005 & 7711 & 52 \\
\hline H (51) & 4140 & -2043 & 7709 & 70 \\
\hline $\mathrm{H}(52)$ & 5917 & -1533 & 7288 & 72 \\
\hline H (53) & 5766 & 61 & 6883 & 62 \\
\hline $\mathrm{H}(54)$ & 3802 & 1126 & 6915 & 46 \\
\hline $\mathrm{H}(56)$ & 356 & 787 & 8816 & 53 \\
\hline $\mathrm{H}(57)$ & -990 & -37 & 9558 & 64 \\
\hline $\mathrm{H}(58)$ & -2039 & -872 & 9030 & 72 \\
\hline $\mathrm{H}(59)$ & -1766 & -886.9999 & 7755 & 82 \\
\hline $\mathrm{H}(60)$ & -416 & -68 & 7003 & 61 \\
\hline $\mathrm{H}(61 \mathrm{~A})$ & -1620 & 7364 & 5220 & 53 \\
\hline $\mathrm{H}(61 \mathrm{~B})$ & -2234 & 6474 & 5349 & 53 \\
\hline $\mathrm{H}(62 \mathrm{~A})$ & 3303 & 1762 & 9253 & 77 \\
\hline $\mathrm{H}(62 \mathrm{~B})$ & 3339 & 1400 & 8457 & 77 \\
\hline $\mathrm{H}(62 \mathrm{C})$ & 3306 & 1136 & 8502 & 77 \\
\hline $\mathrm{H}(62 \mathrm{D})$ & 3123 & 1789 & 9185 & 77 \\
\hline
\end{tabular}

